University of Tennessee Health Science Center

UTHSC Digital Commons

\title{
Effects of Cocaine Sensitization on Drug Self-Administration, Mesocorticolimbic SAPAP Levels, and Prefrontal lonotropic Glutamate Receptors
}

Kyle Christopher Summers

University of Tennessee Health Science Center

Follow this and additional works at: https://dc.uthsc.edu/dissertations

Part of the Neurosciences Commons, and the Substance Abuse and Addiction Commons

\section{Recommended Citation}

Summers, Kyle Christopher , "Effects of Cocaine Sensitization on Drug Self-Administration, Mesocorticolimbic SAPAP Levels, and Prefrontal lonotropic Glutamate Receptors" (2015). Theses and Dissertations (ETD). Paper 264. http://dx.doi.org/10.21007/etd.cghs.2015.0306. 


\title{
Effects of Cocaine Sensitization on Drug Self-Administration, Mesocorticolimbic SAPAP Levels, and Prefrontal Ionotropic Glutamate Receptors
}

\author{
Abstract \\ Towards the goal of improving the knowledge base of how drugs of abuse function to create addicts and \\ using currently uninvestigated areas of this knowledge base, we focused our research studies the male \\ albino rat, and strived to explain how cocaine sensitization alters particular molecular mechanisms in the \\ mesocorticolimbic system related to glutamate receptors, SAPAPs, and affects drug self-administration. \\ Taking all the previously discussed research studies into consideration, we hypothesized that low-dose \\ cocaine self-administration would yield a significant elevation in drug seeking behavior for \\ psychostimulant sensitized animals. For specific changes at the PSD, we hypothesized that acute cocaine \\ exposure and/or cocaine sensitization would alter iGluRs levels in the MPFC, and SAPAP levels in multiple \\ sites of the mesocorticolimbic system. To test these hypotheses, we investigated the effects of \\ psychostimulant sensitization on various parameters of self-administration by infusing a lowdose \\ cocaine reward proven to not induce sensitization during self-administration (reported here as $0.3 \mathrm{mg} / \mathrm{kg} /$ \\ infusion). Although drug exposure did not significantly alter self-administration behavior, we did find that \\ more drug exposed subjects acquired self-administration behavior when compared to drug naïve \\ controls. We also investigated the effects of acute cocaine exposure and/or cocaine sensitization on \\ iGluRs in the mPFC and SAPAPs in the entire mesocorticolimbic circuit thru western blotting, \\ immunolabeling of proteins of interest, and comparing protein levels to those found in cocaine naïve \\ controls. A limited self-administration protocol also tested if SAPAP levels were altered by self- \\ administration of a low-dose cocaine reward. For these experiments, we found that SAPAP protein levels \\ are altered in multiple regions of the mesocorticolimbic system in response to contingent and non- \\ contingent cocaine exposure, and that iGluR receptor subunits are altered in the prefrontal cortex in \\ response to noncontingent cocaine exposure.

\section{Document Type} \\ Dissertation \\ Degree Name \\ Doctor of Philosophy (PhD) \\ Program \\ Biomedical Sciences \\ Research Advisor \\ Jeffery D. Steketee, Ph.D \\ Keywords \\ Addiction, Cocaine, Glutamate Receptors, SAPAP, Self-Administration, Sensitization

\section{Subject Categories} \\ Medical Sciences | Medicine and Health Sciences | Mental and Social Health | Neurosciences | Substance \\ Abuse and Addiction
}




\title{
Effects of Cocaine Sensitization on Drug Self-Administration, Mesocorticolimbic SAPAP Levels, and Prefrontal Ionotropic Glutamate Receptors
}

\author{
A Dissertation \\ Presented for \\ The Graduate Studies Council \\ The University of Tennessee \\ Health Science Center
}

\author{
In Partial Fulfillment \\ Of the Requirements for the Degree \\ Doctor of Philosophy \\ From The University of Tennessee
}

By

Kyle Christopher Summers

August 2015 
Copyright (C) 2015 by Kyle Christopher Summers. All rights reserved. 


\section{DEDICATION}

To my mother Andrea, who through her example taught me that the values of hard work, integrity, and good manners never go out of style

To Karen, who taught me the true meaning of courage under the threat of certain death, and that those no longer with us physically continue to live in our thoughts 


\section{ACKNOWLEDGEMENTS}

All information and data in this text was only possible due to the generous support and inexhaustible patience of my advisor Jeff Steketee and my graduate committee (which included Drs. John Boughter, Michael McDonald, Kazuko Sakata, and Wen Lin Sun). Also, instrumental to the completion of this project were the helping hands of Dr. Steve Tavalin. This project also could have never been completed without financial support from the University of Tennessee Health Science Center Neuroscience Institute and the National Institute on Drug Abuse (NIDA). 


\begin{abstract}
Towards the goal of improving the knowledge base of how drugs of abuse function to create addicts and using currently uninvestigated areas of this knowledge base, we focused our research studies the male albino rat, and strived to explain how cocaine sensitization alters particular molecular mechanisms in the mesocorticolimbic system related to glutamate receptors, SAPAPs, and affects drug self-administration. Taking all the previously discussed research studies into consideration, we hypothesized that low-dose cocaine self-administration would yield a significant elevation in drug seeking behavior for psychostimulant sensitized animals. For specific changes at the PSD, we hypothesized that acute cocaine exposure and/or cocaine sensitization would alter iGluRs levels in the mPFC, and SAPAP levels in multiple sites of the mesocorticolimbic system. To test these hypotheses, we investigated the effects of psychostimulant sensitization on various parameters of self-administration by infusing a low-dose cocaine reward proven to not induce sensitization during self-administration (reported here as $0.3 \mathrm{mg} / \mathrm{kg} /$ infusion). Although drug exposure did not significantly alter self-administration behavior, we did find that more drug exposed subjects acquired selfadministration behavior when compared to drug naïve controls. We also investigated the effects of acute cocaine exposure and/or cocaine sensitization on iGluRs in the MPFC and SAPAPs in the entire mesocorticolimbic circuit thru western blotting, immunolabeling of proteins of interest, and comparing protein levels to those found in cocaine naïve controls. A limited self-administration protocol also tested if SAPAP levels were altered by self-administration of a low-dose cocaine reward. For these experiments, we found that SAPAP protein levels are altered in multiple regions of the mesocorticolimbic system in response to contingent and non-contingent cocaine exposure, and that iGluR receptor subunits are altered in the prefrontal cortex in response to non-contingent cocaine exposure.
\end{abstract}




\section{PREFACE}

The Incans that first cultivated the coca plant for medicinal use five millennia ago reserved this "Elixir of the Gods" for priests, social elites and 'pure' virgin sacrifices. In more modern times, cocaine has even been believed to be the cure to cancer and called the "champagne of drugs". Yet today, cocaine use (especially in the crystallized free-base form of "crack") is associated with the dregs of society. As the complexities (and the associated evils) of our modern society increases, so seems the prevalence of addictions of all varieties.

Cocaine addiction is defined as a long-lasting disorder marked by chronic and compulsive cocaine use, resulting in neurobiological changes. Behavioral, these changes are characterized by a loss of control over use, eventual tolerance, and social isolation. If untreated, death from some facet of drug use usually occurs. Unfortunately, very few treatments options exist, and even fewer with high rates of success. Currently, none of these more successful treatments options include targeted pharmacological therapy. In an effort to rectify this, the development and research of treatment options are a priority for pharmaceutical companies and are constantly evolving. Although especially prevalent in the United States, cocaine addiction has reached epidemic levels worldwide. Cocaine addiction does not discriminate by social class: addicts range from those in poor innercity communities, to those working on Wall Street. This widespread use is possibly because of social factors that foster maladaptive behaviors, and possibly even environmental contamination resulting from inappropriately discarded industrial waste.

The effects of cocaine addiction are not restricted to biological factors, or even solely to cocaine users. It is a fact (although one that may not fit the widely accepted narrative) that most cocaine users only use recreationally, and do not develop an addiction. As of 2008, the National Institute on Drug Abuse (NIDA) reported that approximately 16 million people between the ages of 15 and 64 years worldwide had used cocaine at some point in their lives. Roughly half of this number (7.1 million) is represented by North Americans. Yet, this same year, it was reported that only 1.4 million people ( $\sim 3 \%$ of Americans) met the clinical requirements under DSM-IV to be diagnosed as having a dependence on/ an addiction to cocaine. Among this minority who transition from recreational use to addiction, compulsivity accompanies drug use. These compulsive features of addiction include a perceived lack of control over drug use, and continued use despite negative consequences associated with addiction. These compulsive symptoms may be related to the impairment of executive function in drug users, as addicts who are diagnosed with high levels of compulsivity also are less likely to persevere in accomplishing long term goals. This same study also found that impulsivity also was likely associated with cocaine use, as addicts made decisions with less forethought. A compulsivity surrounding drug use is also suggested by the finding that cocaine addicts prefer to use drugs in one specific place. This culmination of enhanced compulsivity and impulsivity in cocaine addicts may also become expressed in ways not associated with drug use, as cocaine addicts tends to crave foods with more fat and carbohydrates than the general public. 
These few who make drug use an all-encompassing lifestyle are a substantial percentage of mentally ill drug users, those with emergency hospital admissions, and criminal arrestees. In fact, cocaine intoxication among arrestees is a significant problem according to The Office of National Drug Control Policy. In its annual Arrestee Drug Abuse Monitoring Program (ADAM) II Report, the Office of National Drug Control Policy reports that $19 \%$ of arrestees in Chicago (25\% in New York City) were under the influence of cocaine at the time of their arrest in 2012. These arrestees can present a job hazard to law enforcement officers by exhibiting hyper-aggressive behavior. This behavior may indicate an underlying medical disorder caused by prolonged cocaine use known as Excited Delirium (more commonly, "cocaine psychosis"). Cocaine psychosis is a collection of symptoms that manifests with delirium, anxiety, hallucinations, psychomotor agitation, elevated temperature/heart rate, and superhuman strength/resistant to pain. Fatal cocaine overdose is commonly preceded by cocaine psychosis, and occurs most commonly due to respiratory paralysis.

Interest from law enforcement extends beyond the scope of individual users to those profiting most from the cocaine economy; the sellers. Although seizures of illicit cocaine imports to US has fallen in recent years, demand for the substance and associated crimes have not. This phenomenon is likely not due to crimes-of-opportunity committed by a large group of people, but the intense motivation of small numbers. Consider the example of burglary in a residential neighborhood. According to the Memphis Police Department, when there is a "rash" of home robberies in a neighborhood, many times these crimes completely stop for the area after a single perpetrator is apprehended. This is because usually instead of many people committing small fractions of the crime, there was one person committing them all. Ironically, cocaine use and property crimes are strongly correlated.

With this example in mind, it is no surprise that the architects in this illicit drug trade also consist of a relatively small number of people. The plant from which cocaine is extracted, Erythroxylon coca, is native to and grows solely at high elevations in the relatively poor countries of Bolivia, Columbia, and Peru. This gives any traffickers of cocaine in these regions a virtual monopoly on sales due to the fact that cocaine is traditionally much easier to extract from the plant itself than to synthetically produce. Moreover, the United States is the most lucrative market for vendors, geographically close to these regions, and has multiple routes for smugglers to exploit due its expansive/largely unrestricted border. Sales in the United States also provides a high return on initial investment due to the wealth of the American consumer when compared to those in many of the surrounding countries. This is evidenced by the fact that as of 2014, a single "uncut" kilogram of cocaine in America can sell for an average of \$30,500. This is price is verified by local law enforcement in Memphis, Tennessee, as the same kilogram can be sold locally for anywhere between $\$ 25,000$ and $\$ 31,000$. In Bolivia, the same kilogram can be purchased for $\$ 800$ to $\$ 1,500$ which translates to at least a hefty $1,600 \%$ return on the initial investment. 
This black market economy is so pervasive and successful that the website for the iconic business publication The Economist devoted an entire section to the economic aspects of cocaine use and trafficking. It is befitting that this publication is interested in cocaine, because not only are high levels of cocaine commonly found on our paper currency, but because this cocaine economy has attracted the attention of world governments and organizations. For example, the United States spends upwards of \$35 billion annually on drug control efforts. In a global targeted response, a UN taskforce has been dedicated to the eradication of the drug trade, and the violent efforts of criminals to monopolize its production and sale. Cross-border cooperation efforts are also coordinated between governments (i.e. Mexico and the United States) to incapacitate "cartels" responsible for cocaine production. This cooperation includes kinetic action operations and the seizure of cartel assets on both sides of the border. Two factors that must be examined to understand the motivations of any organization (legal or illicit) is ease of access to raw materials, and the risk/reward ratio. Considering that the qualities that cocaine imparts can become quite tempting to users, any rewards are greater than risks to importers. 


\section{TABLE OF CONTENTS}

CHAPTER 1. INTRODUCTION .................................................................................

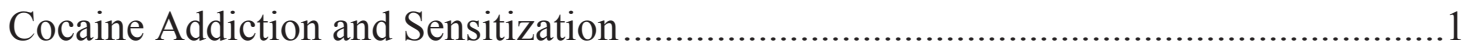

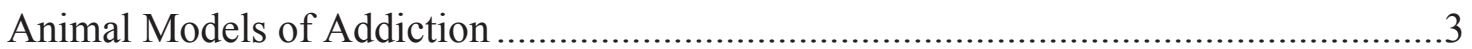

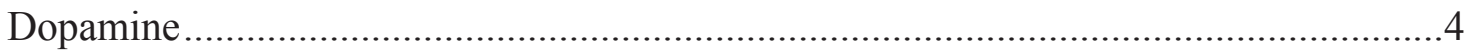

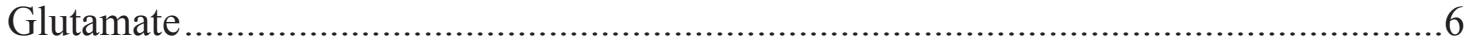

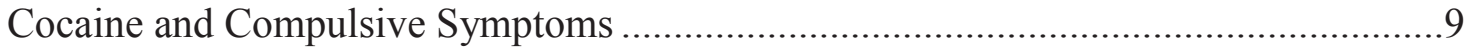

\section{CHAPTER 2. EFFECTS OF SENSITIZATION ON COCAINE SELF-}

ADMINISTRATION .................................................................................................12

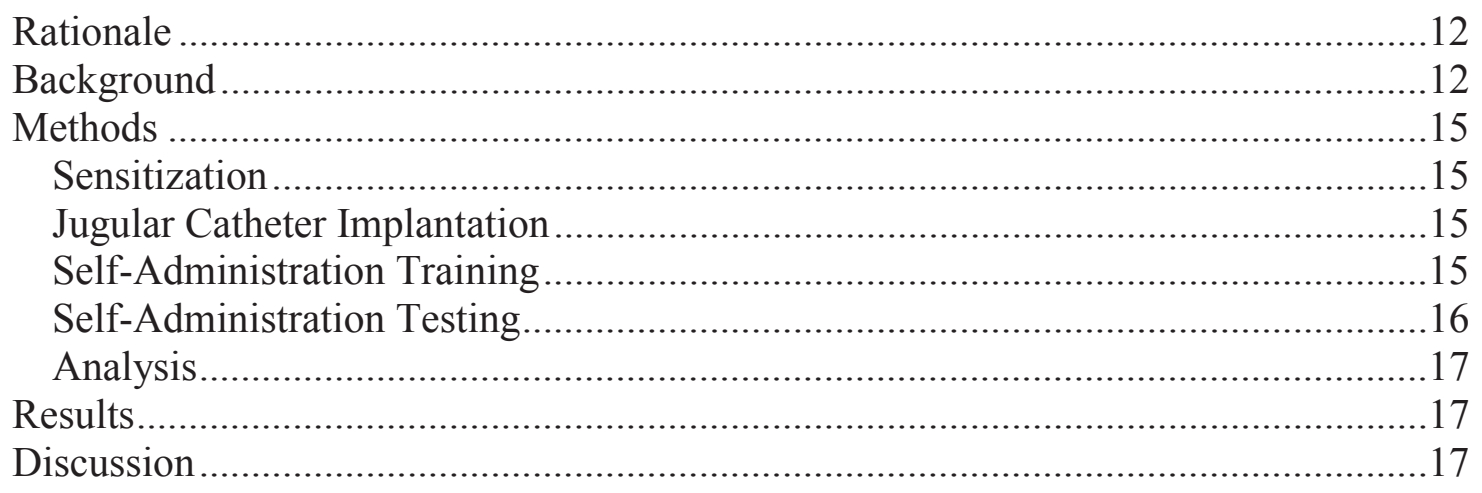

\section{CHAPTER 3. EFFECTS OF COCAINE SENSITIZATION ON SAPAP}

LEVELS IN THE MESOCORTICOLIMBIC SYSTEM ........................................22

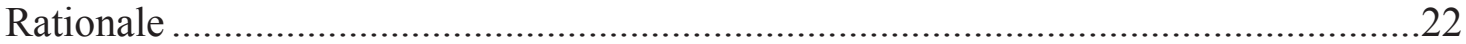

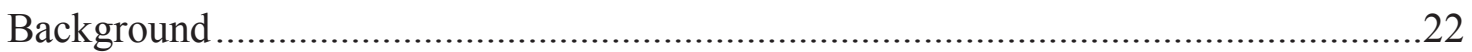

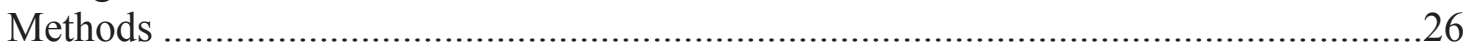

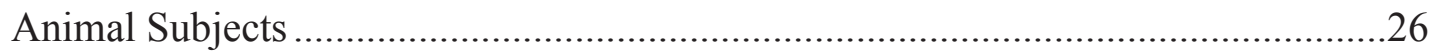

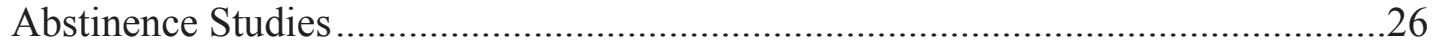

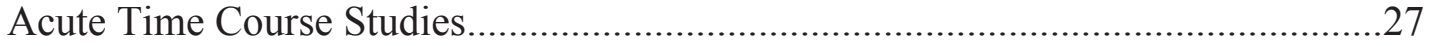

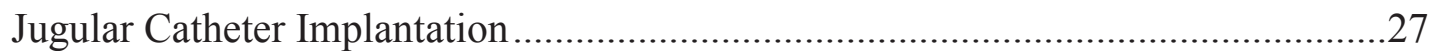

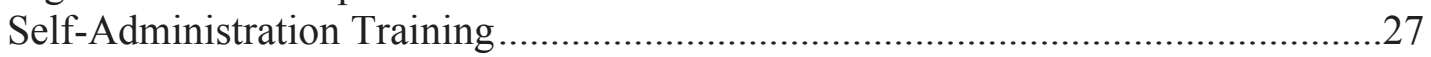

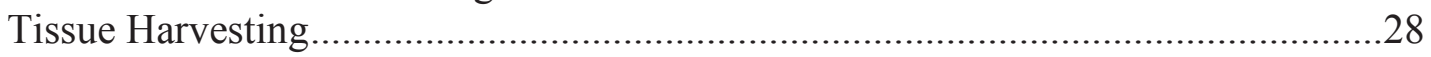

Initial Preparation, Protein Assay, and Immunoblotting ...................................28

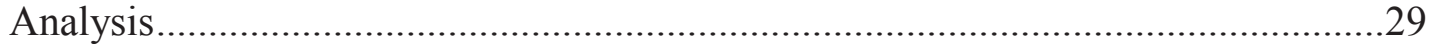

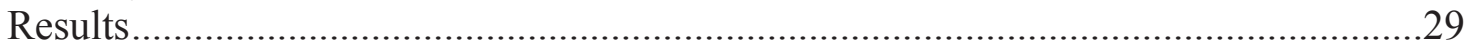

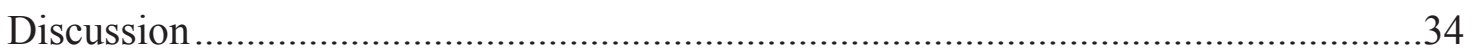

\section{CHAPTER 4. SENSITIZATION-INDUCED CHANGES OF AMPA/NMDA}

RECEPTOR RATIO IN THE MPFC ....................................................................41

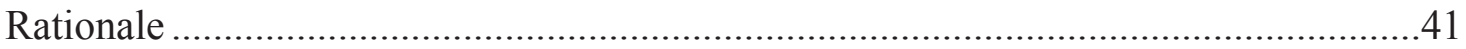

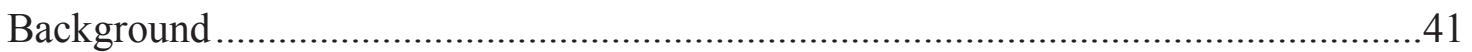

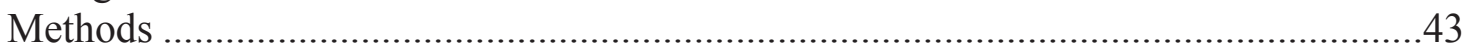

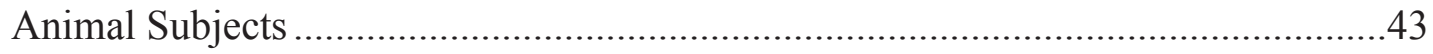




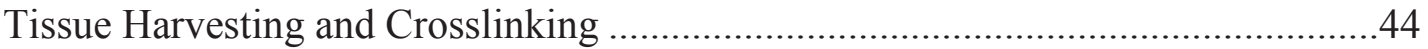

Protein Sample Preparation and Immunoblotting .................................................4

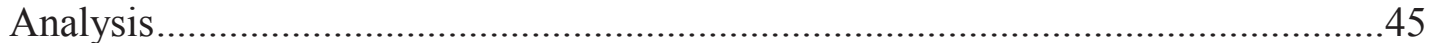

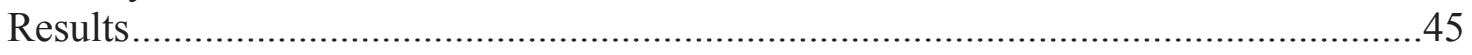

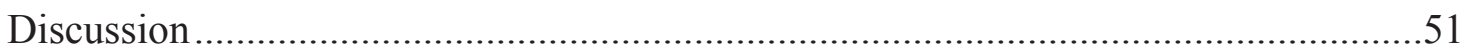

CHAPTER 5. CONCLUSION ...............................................................................57

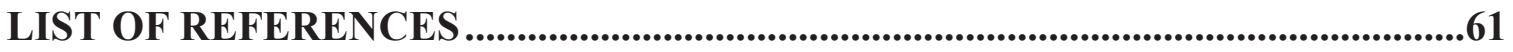

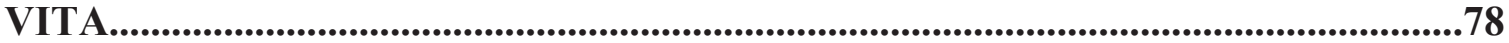




\section{LIST OF TABLES}

Table 3-1. Acute SAPAP Changes in Areas Other Than the mPFC .............................33 


\section{LIST OF FIGURES}

Figure 1-1. Diagram of Sensitization Related Areas in the Rat Brain.............................5

Figure 1-2. General Structure of iGluRs and mGluRs. ...........................................

Figure 2-1. Graphic Flowchart for Self-Administration Protocol. ................................18

Figure 2-2. Sensitization Verification by Infrared Home Cage Monitors. ......................19

Figure 2-3. Chi Square Analysis Comparing Treated and Untreated Animals During

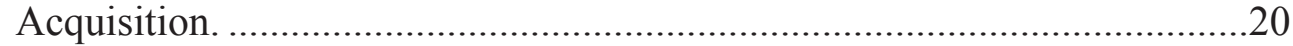

Figure 3-1. Acute Cocaine Exposure Induces a Transient Reduction in Prefrontal SAPAP Levels.

Figure 3-2. Comparisons of Various Acute Time Points for SAPAPs 1 through 4 in the Dorsal and Ventral mPFC.

Figure 3-3. Sensitization Verification by Infrared Home Cage Monitors.

Figure 3-4. Varied SAPAP Reduction in Dorsal mPFC at 3 Weeks. .36

Figure 3-5. SAPAP Levels of the Dorsal mPFC Are Elevated for All SAPAPs in Test Self-Administration Rats.

Figure 4-1. Sensitization Verification by Infrared Home Cage Monitors. . .46

Figure 4-2. AMPA Receptor Changes at 2-Weeks...................................................48

Figure 4-3. GluR2 Subunit Changes in the Ventral mPFC at 3-Weeks.

Figure 4-4. NR1 and NR2A Subunit Changes at 2-Weeks. .50

Figure 4-5. Surface NR2A Subunit Changes at 2-Weeks in the Ventral mPFC. 52

Figure 4-6. NR2B Subunit Changes at 2-Weeks. 53

Figure 4-7. NR2B Subunit Changes in the Ventral mPFC at 3-Weeks. 54

Figure 5-1. Possible Correlation Between SAPAP and AMPA/NMDA Receptor Studies. 


\section{LIST OF ABBREVIATIONS}

$\begin{array}{ll}\text { AMPA } & \alpha \text {-amino-3-hydroxy-5-methyl-4-isoxazolepropionic acid } \\ \text { DAT } & \text { dopamine transporter } \\ \text { ERK } & \text { extracellular signal regulated kinases } \\ \text { FR } & \text { fixed ratio } \\ \text { HPA } & \text { hypothalamus-pituitary-adrenal } \\ \text { iGluR } & \text { ionotropic glutamate receptor } \\ \text { mGluR } & \text { metabotropic glutamate receptor } \\ \text { mPFC } & \text { medial prefrontal cortex } \\ \text { Nacc } & \text { nucleus accumbens } \\ \text { NMDA } & \text { N-methyl-D-aspartic acid } \\ \text { PR } & \text { progressive ratio } \\ \text { SAPAP } & \text { synapse associated protein } 90 \text { associated protein } \\ \text { VTA } & \text { ventral tegmental area }\end{array}$




\section{CHAPTER 1. INTRODUCTION}

\section{Cocaine Addiction and Sensitization}

Cocaine users report that cocaine imparts a feeling of euphoria, as well as increased endurance, stamina, and resistance to fatigue. Indeed, these qualities are virtually verbatim extolled by Sigmund Freud, who prescribed it to his patients and struggled with cocaine dependence personally [1]. In his 1884 manuscript in support of cocaine, Über Coca, he described the substance as "[inducing] the most gorgeous excitement..... [warding] off hunger, sleep, fatigue, and steels one to intellectual effort" [2]. Despite Freud's support earlier in his career, cocaine use can be dangerous. Interestingly, not only is this alkaloid a naturally occurring neurotoxic pesticide [3], but also has a high addiction potential.

Death from cocaine overdose can often be disturbing to observe [4, 5]. Scientists strive to explain why addicts willingly use cocaine, with possible psychological explanations including modeling cocaine use as a learned behavior that serves as an 'escape hatch' to the prevention of/solution to emotional distress [6]. Another theory posits that small minority of users that become addicted may be experiencing a perfect storm of drug exposure, social reinforcement, environmental priming [7] and genetic predisposition [8]. Additional studies even indicate that those who have lost all perceived control over their drug use usually report chronic stress/ abuse during their childhoods and adolescences [9]. While not necessarily directly responsible for their drug use, these events may act as precipitating factors. As evidence in animal models, juvenile rats display behavior reminiscent of cocaine sensitization upon their initial exposure to cocaine if exposed to repeated, intermittent, and uncontrollable foot shocks. In this "stress sensitization" theory, stress acts a causative agent in the sensitization process in place of cocaine. Similarly, both agents require repeated, intermittent exposure and activate the HPA axis-centered stress response ([10], review). While this manuscript does not discount these factors, our research group also contends that the early effects of cocaine itself may contribute equally, if not more, to the development of cocaine dependence. The initial effects of cocaine use, while reported to be pleasurable, may also result in cocaine sensitization. Cocaine sensitization is defined as the ability of repeated exposure to psychostimulants, such as cocaine or amphetamine, to result in lifelong druginduced neurophysiologic modifications. The hallmark of sensitization is the ability of a specific dose of a psychostimulant to elicit higher locomotor responses upon repeated administration [11].

Behavioral expression of cocaine sensitization is caused by distinct molecular and anatomical modifications due to long term drug exposure. Cocaine sensitization is also believed to be responsible for socially-disturbing aspects of addiction, including the cocaine-induced psychosis and its associated risks, relapse despite years of abstinence, and intrusive thoughts of drugs/drug use. Although traditionally associated with animal studies, there is evidence that sensitization occurs in humans, resulting in similar behavioral and molecular consequences. Although not well understood, this phenomenon 
is not new to the scientific world; the earliest reports of psychosis can be dated to the late 19th century [12]. In fact, the Incans also reported that the effects of cocaine increase over days of use, a phenomena that researchers at the time described as "increase in reflex excitability" paired with increased "gayness" [13]. Sensitization, by the nature of the phenomenon, is practically impossible to observe in human addicts, and has long been a topic on controversy among researchers of addiction [14].

The crux of the argument against cocaine sensitization stems from the difficulty of observing its development in humans. Direct observation is virtually impossible since it would occur very early in the addiction process before addicts would usually seek professional help to control their addiction. In addition to the aforementioned historical anecdotes, there are however very compelling studies that suggest its existence in humans. For example, the repeated and intermittent doses of a stimulant that acts through similar mechanisms as cocaine (methamphetamine) increases the eye blink, heart rate, and subjective feeling of euphoria in drug naïve volunteers $[15,16]$. Cocaine addicts also tend to display repetitive and seemingly non-productive movement patterns, which is similar to stereotypy observed in sensitized rats after repeated exposure to drug [17-19].

Yet, no matter how debilitating and immense the challenges against current drug users, it could be argued that the most insurmountable challenges begin after use ends. One of the most challenging aspects of addiction is the phenomenon of relapse, which can occur after months or even years of complete drug abstinence. Addicts in relapse often cite a wide variety of common world objects eliciting a strong and seemingly uncontrollable craving [20] (i.e. walking past a bar if an alcoholic). Based on these reports from addicts, relapse behavior is also commonly studied in rodent models as cue induced reinstatement. Rats exposed intermittently to cocaine can be trained to selfadminister drug in conjunction with a cue light activated when cocaine is administered. When extinguished, rats will reinstate their drug-seeking behavior when presented with a cue light, often after long periods of forced abstinence [21].

Sensitization changes on a smaller scale in model organisms are responsible for the maintenance of operant responding for drug [22], and consist of neuronal changes at the molecular [23] and anatomical [24] level. Even though many of the molecular events leading up to, and that occur during sensitization are poorly understood, it is seemingly permanent. As evidence, these changes persist even after a full year of abstinence in response to a systemic challenge dose in rodent models [25]. This is a significant time period considering rats rarely live this long in the wild, and typically live approximately 2 years under laboratory conditions [26]. Sensitization almost certainly plays a role in the maintenance of self-administration, although few self-administration studies have examined the direct effects of sensitization on drug acquisition. There have not been any published studies that proposed a model using a dose high enough to stimulate an animal to respond, yet low enough to avoid inadvertent sensitization of saline controls.

When examining addiction using the earlier outlined features of cocaine addiction, certain facets of this disease could be considered subjective. For example, how much loss of control over use must be observed to qualify for addiction? Exactly what 
defines social isolation? Although the exact scale of these features may vary qualitatively, the neurobiological changes can be defined in a clear quantitative manner. Although important to a global understanding, in this manuscript we limit our discussion of addiction to the neurobiological plasticity caused by cocaine exposure. On this front, a wealth of human data indirectly implicates various neurotransmitter systems in response to chronic cocaine exposure. Yet, animal models are arguably a more useful tool in dissecting the exact molecular, anatomical, and physiological effects caused by cocaine exposure. Our model organism of choice is the rat, considering the relatively low cost of maintenance, small storage footprint, the ability to conduct invasive procedures impossible in human beings, and the fact that the rat model has a long history of use in addiction studies, and will respond for cocaine under operant training [27].

\section{Animal Models of Addiction}

The Science of Making Drug Addicted Animals by S.H. Ahmed [7] goes into great detail about the social and neurological factors that may lead to drug abuse, and how these factors can be reliably reproduced in rats. Concentrating this work to its most basic levels, we uncover several important themes. Similar to clinical literature, animal studies involving rats prove that many animals show resilience against cocaine acquiring self-administration. As evidence, most rats have a strong preference to saccharin-fortified

"sweet water" over a cocaine reinforcer even following extended access to cocaine. Yet if lacking an alternative choice under self-administration, almost all animals will respond for a cocaine reward in an operant chamber [7]. However, when given the choice to either respond for sweet water or cocaine, very few animals continue to prefer drug rewards. Indeed, out of 184 rats examined using self-administration studies over 5 years, only 16 animals (termed 'high responders') prefer cocaine over the sweetened water. This result is not due to saccharin aversion, since when given access to sweet water alone, these rats consumed similar amounts to non-cocaine preferring rats [7].

Parallel to clinical studies, an escalation of drug use is observed in rats given extended access to drug. These same animals also show increased motivation to selfadminister cocaine. As evidence, extended access to cocaine is associated with increased break points under progressive ratio (PR) testing, higher break points over control animals with sham infusions, and higher break points during self-administration with a cue previously associated with pain. Additionally, rats will continue to self-administer cocaine even when deprived of food and given a choice of hunger-abating water with sucrose added, a result that the author attributes to the compulsive nature of runaway drug use [7]. Drug preferring animals and humans also both display parallel systemic effects of cocaine, especially in relation to its effects on cardiac function and stresspriming. These topics are beyond the scope of this text, and are treated in through detail in other published manuscripts [28-31]. 


\section{Dopamine}

However, experiments in animal models have shown that cocaine acts through a fairly simple mechanism of action of monoamine reuptake inhibition. This subject is well within our scope, especially since this mechanism precipitates cocaine sensitization. Cocaine induced changes in monoamine levels are caused by cocaine's targeting of monoamine transporters [32]. These transporters are responsible for removing neurotransmitters from the synaptic cleft after presynaptic release [33], and are responsible for the most common effects of the drug when inhibited by cocaine [34]. While cocaine blocks all monoamine transporters to some degree [35,36], its main action responsible for the reinforcing properties of cocaine is blockade of the dopaminergic transporter (DAT) [37-39]. As evidence, animals trained to self-administer cocaine will self-administer the specific DAT inhibitor PTT at similar levels to cocaine [40]. This DAT blockade, while pan-neuronal in nature, differentially affects discrete regions of the mesocorticolimbic system in the sensitized brain [11,41]. Although increased locomotion is the main phenotypic expression of sensitization, this motor effect "saturates" in contrast to the perceived reinforcing effects of cocaine which continue to escalate as exposure increases [42]. This escalation may be the main motivator that drives addiction.

Sensitization can be modeled by two distinct phases that involve the ventral tegmental area (VTA) in the midbrain, and the nucleus accumbens (Nacc) and medial prefrontal cortex (mPFC) in the forebrain (Figure 1-1). The first is associated with the VTA, which precipitates secondary phase in the Nacc. This first VTA-centered phase can be described as initiation (or incubation), during which cocaine exposure directly causes an increase in synaptic dopamine by DAT blockade, which in turn causes immediate transient effects. During incubation, while cocaine induces intense feeling of euphoria, the behavioral hallmarks of sensitizations are not present. As DAT blockade-induced molecular events began developing, output from the VTA to the Nacc is altered. As evidence for this incubation theory, blocking all protein synthesis in VTA can completely block the behavioral effects of sensitization [43]. Moreover, this study also found intracranial injections of cocaine directly in to the VTA but not the Nacc of drug naïve rats leads to behavioral sensitization along with its molecular hallmarks in the Nacc. Through altered input from the VTA in the initiation phase by dopaminergic "A10" neurons to the Nacc, the secondary phase of sensitization develops [44]. During expression, increased output from medium spiny neurons in the Nacc to the motor cortex results in enhanced motor responses. It is important to note that this process of expression is delayed when compared to the immediate effects of initiation. Considering neurotransmitters systems act in concert to regulate behavior, increases in dopamine in a discrete area such as the VTA has far reaching implication to a wide variety of brains areas and neurotransmitter systems [45]. In this manuscript, we will focus on how glutamate transmission is altered in the $\mathrm{mPFC}$ by cocaine exposure. 


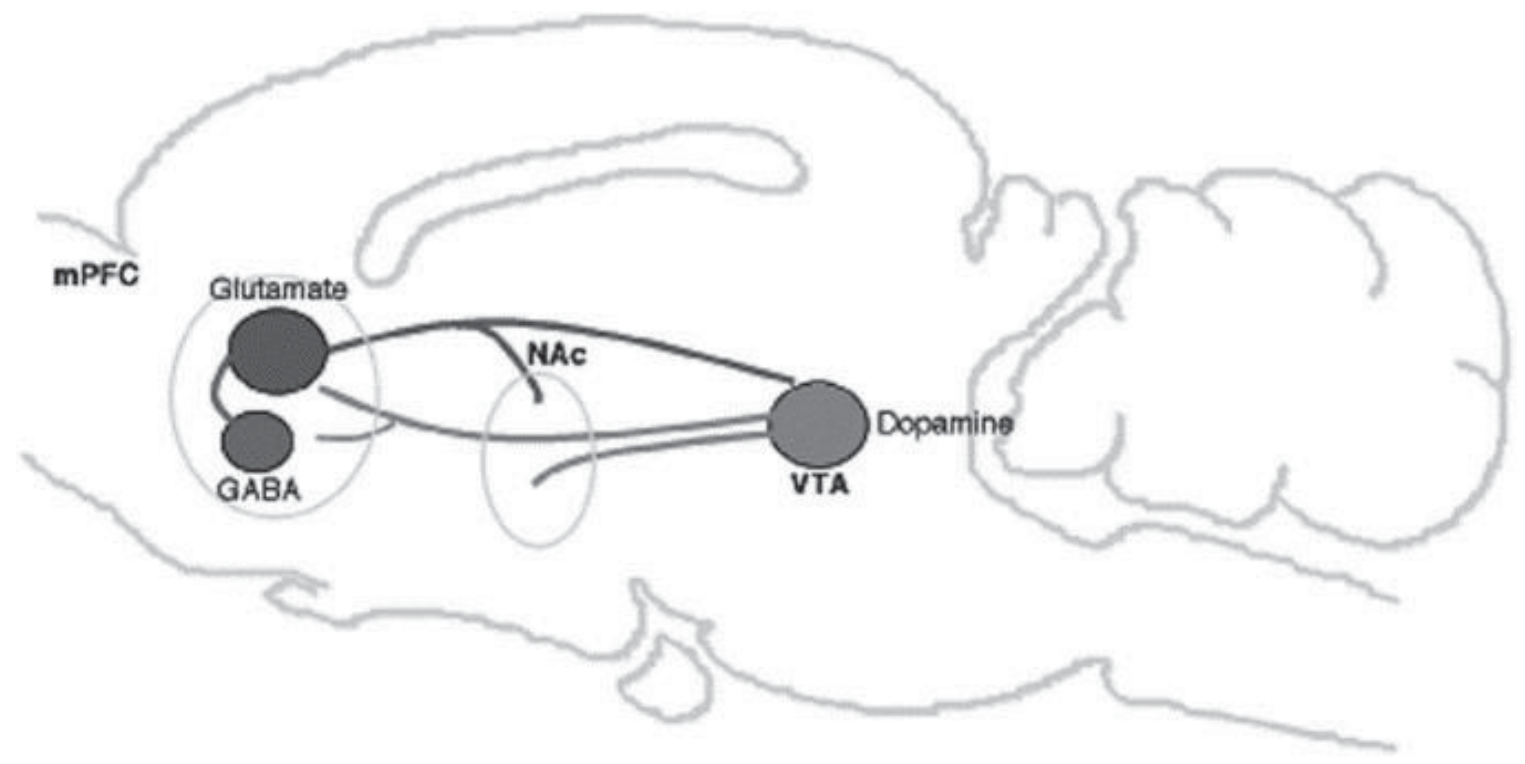

Figure 1-1. Diagram of Sensitization Related Areas in the Rat Brain.

Diagram of the location of key brain areas involved in sensitization and neurotransmitter projections. Dopamine originates in the VTA and projecting to the Nacc and mPFC. Glutamate originates in the mPFC and projects to the Nacc and VTA. Reprinted from Brain Research Reviews, volume 41/issue 2-3, Jeffrey D Steketee, "Neurotransmitter systems of the medial prefrontal cortex: potential role in sensitization to psychostimulants", Pages No. 203-228, 2003, with permission from Elsevier [46]. 


\section{Glutamate}

The prefrontal cortex is heavily modified by drug use, and is the source of glutamate to other regions of the mesocorticolimbic circuit. In vitro application of dopamine transiently depresses prefrontal glutamatergic synaptic responses in cocaine naïve rats, which is extended when paired with tetanic stimulation [47]. Additionally, cocaine increases extracellular dopamine levels in the prefrontal cortex of acutely exposed animals [48]. After 7 days of cocaine abstinence, markers of reduced synaptic activity are observed including reduced prefrontal dendrite density and spine number [49]. These findings suggest that increase dopamine levels observed due to early cocaine exposure causes suppression of synaptic activity in the prefrontal cortex at later time points. This effect may be evidenced in addicts by the fact that live addicts show functional "lesions" in fMRI scans in the prefrontal cortex [50]. Furthermore, prefrontal cortical lesions have been shown to affect inhibitory dopaminergic D2 receptors in the Nacc [51] which may have functional consequences for relapse behavior [52]. As a result, current literature implicates glutamate as occupying an increasingly more important role in the processes of drug-induced euphoria, psychosis, craving, and relapse. This also links glutamate to cocaine-induced behavioral changes. In addition, specific prefrontal areas such as the anterior cingulate gyrus and the lateral prefrontal cortex show reduced function in abstinent cocaine abusers that is negatively correlated with increased cocaine exposure. This is an important finding, as the connection between these two areas is associated with making decisions involving intense mental effort and choosing an atypical response (drug abstinence) over a habitual one (continued drug abuse) [53].

In addition to hypofunctional areas in the medial prefrontal cortex (mPFC) and morphology changes in individual neurons, cocaine exposure causes changes on a smaller-scale affecting receptor population and receptor function. These changes are well-documented in animals exposed to cocaine in other regions of the mesocorticolimbic system. Yet, while prefrontal glutamate is a clear mediator of cocaine-induced behavior, any effects of cocaine sensitization on ionotropic glutamate receptors (iGluRs) have yet to be examined in the prefrontal cortex. These ionic receptors are the main mediators of glutamatergic effects in the adult brain, and are split between two main classes of receptors, consisting of multiunit complexes that join together to form a membranespanning cation-permeable pore (Figure 1-2). Examined for selective agonism, iGluRs are sensitive to either $\alpha$-amino-3-hydroxy-5-methyl-4-isoxazolepropionic acid (AMPA) or to N-methyl-D-aspartic acid (NMDA). This preference is determined by the spacing between the amino and carboxylate groups of each subunit [54].

The effects that are responsible for glutamate being the main excitatory neurotransmitter in the brain are mediated by ionotropic AMPA-sensitive [55] glutamate receptors, and are often referred to as "excitatory glutamate receptors" in older literature as a reflection of this fact $[56,57]$. Structurally, these receptors consist of four poreforming subunits of two subunits types, a configuration that could be envisioned as a dimer (heterotetramer) of dimers (2 identical subunits of only GluR1/2/3or 4) [58]. When fully assembled, these receptor serve as major mediators of long-term potentiation, as 


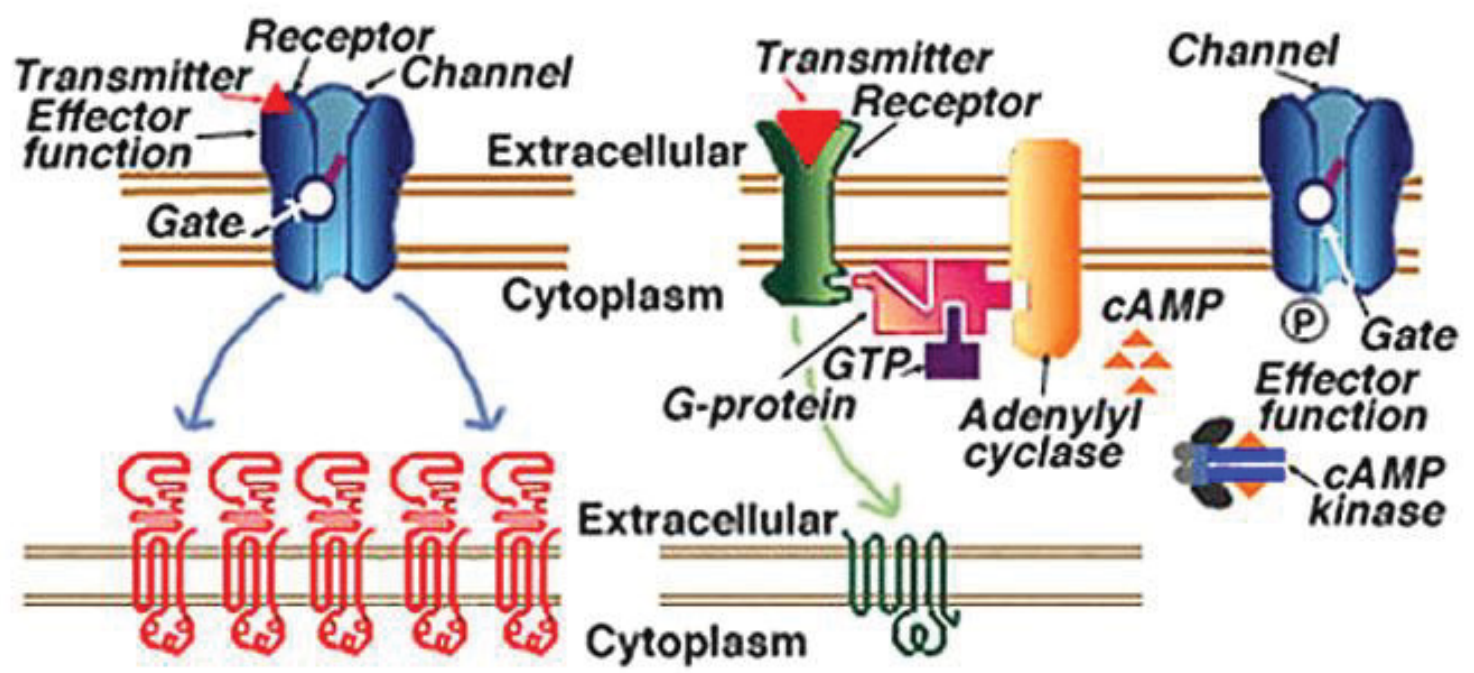

Fig. 5a. Ionotropic receptors and their associated ion channels form one complex (top). Each iGluR is formed from the co-assembly of multiple (4-5) subunits (From Kandel et al., 1991).
Fig. 5b. Metabotropic receptors are coupled to their associated ion channels by a second messenger cascade (top). Each mGluR is composed of one polypeptide, which is coupled to a G-protein (from Kandel et al., 1991).

Figure 1-2. General Structure of iGluRs and mGluRs.

While iGluRs are assembled from several subunits to form a membrane-spanning pore permeable to cations, mGluRs activate signal cascades in response to glutamate. Note that mGluRs can also regulate iGluRs. Reprinted with permission from Webvision. Connaughton, V., Glutamate and Glutamate Receptors in the Vertebrate Retina., in Webvision: The Organization of the Retina and Visual System [Internet], F.E. Kolb H, Nelson R, Editor 2005 May 1 [Updated 2007 May 7], University of Utah Health Sciences Center: Salt Lak City, UT. [59]. 
high frequency stimulation of neurons can result in synaptic strengthening through increasing the ratio of AMPARs to NMDARs [60].

Along with AMPA receptors, NMDA-type glutamate receptors are vital to any discussion of the effects of cocaine exposure. These receptors are key players in the functions of synaptic plasticity and enduring changes in cortical neurons [61]. Low-level stimulation of NMDA receptors leads to synaptic depression (weakening) [62], higher stimulation leads to synaptic potentiation (strengthening) [63], and very high levels leads to cell death [64], all of which are processes dependent on Ca2+ influx. Similar to AMPA-type glutamate receptors, NMDA receptors are voltage-gated, responsive to Lglutamate [65], PSD localized [66], and consist of heterotetrameric units [67]. Mature NMDA receptors contain a constitutive and ubiquitously expressed NR1 subunit and at least one member of the NR2 subunit family (NR2A/B/C/D) confined to more discrete areas [68].

Although not examined in the prefrontal cortex, iGluR populations are misregulated in the remainder of the mesocorticolimbic circuit in response to cocaine sensitization. These effects include transient changes in the VTA that increases dopamine output to the Nacc through enhancement of AMPA receptor currents [69]. In contrast to those in the VTA, sensitization-induced changes in the Nacc appear to be permanent and alter glutamate receptor population and function. These changes in the Nacc cause a regional hyper-excitability due to an increased contribution of $\mathrm{Ca} 2+$-permeable AMPA receptors, but also a decrease in any glutamatergic braking ability by mGlur1 and NMDA receptor input. Considering that the NMDAR subunits NR2A/B are expressed at their highest levels in the cerebral cortex [70], it stands to reason that changes in iGlurR population due to sensitization should be thoroughly investigated in the mPFC. Surprisingly, any prefrontal iGluR population changes in response to cocaine sensitization have yet to be published. Additionally, clinical studies have shown decreased overall activity and glutamate levels in the prefrontal cortex of cocaine addicts even after abstinence [71]. This indicates that prefrontal glutamate receptors could play a vital role in the long term expression of sensitization.

Considering the braking ability of mGluRs cited above, mechanisms affecting the expression of cocaine sensitization appear to include ionic receptor changes as well as intracellular signaling cascades. In addition to their functions of current conduction and similar to mGluRs, iGluRs have the ability to initiate cell signaling through their intracellular domains to regulate synaptic responses. As evidence, NMDA receptors regulate the surface expression of AMPA receptors through the Ras-ERK pathway, with NR2A-containing NMDARs promoting AMPAR expression, and those containing NR2B subunits depressing this expression [72]. AMPA receptors even have the ability to remove themselves from the synapse via the GluR2 subunits through BRAG2-mediated endocytosis [73]. These signaling mechanisms, however, may not only regulate the functional ratio of AMPA/NMDA receptors at the synapse, but could also alter function of receptors present at the synapse. Phosphorylation by PKC of the GluR1 subunit at Ser(831) increases AMPA channel conductance [74]. In addition to these phenomena, cytoskeletal remodeling is known facilitate drug-induced habit formation in an unknown 
fashion [75], and in the case of cocaine sensitization, clear and dramatic morphological changes occur in MSNs of the Nacc [76]. Synapse associated proteins may participate is this process, and splice variants of synapse-associated protein 102 (SAP102) are implicated in synaptic remodeling by subunit-specific regulation of NMDARs by anchoring these receptors to the synapse [77]. Following this finding, although there is not a known cure for addiction, NMDA receptors may be particularly important in ameliorating certain symptoms associated with addiction. Interestingly, NMDAR inhibitor trazodone, a traditional medication for the treatment of symptoms associated with Obsessive Compulsive Disorder (OCD), has been used to successfully reduce compulsive drug-foraging in cocaine addicts, although the mechanism behind this are unknown $[78,79]$.

\section{Cocaine and Compulsive Symptoms}

Although possibly an unorthodox treatment when first implemented for cocaine addicts, closer examination shows that several similarities are common among cocaine abusers experiencing craving ("jonesing") and OCD patients, including unwanted, intrusive, and anxiety-related thoughts [80], and compulsive and repetitive foraging for non-existent drugs. In fact, co-morbid diagnoses of both disorders are not uncommon [81]. Ironically, while trazodone inhibits the activity of ionotropic NMDA receptors, these receptors are linked at the synaptic surface to the underlying cytoskeleton by SAP90 and SAP90 associated proteins (SAPAPs) $[82,83]$. While the SAP90 has been exhaustively studied ([84], review), relatively little is known about its associated SAPAPs. SAPAPs may prove to be an important target in the progression of the compulsive symptoms of cocaine addiction. SAPAPs exist in 4 isoforms (SAPAP 1, 2, 3, and 4), range in size from $90 \mathrm{kDa}-120 \mathrm{kDa}$ [85], and serve as the intermediary between SAP90 at the PSD and the underlying cytoskeleton [86]. All SAPAPs have a conserved guanylate kinase domain that is thought to activate NMDA receptors [87], and are localized to the soma with the exception of the dendritically localized SAPAP3 [85]. SAPAP2 (also known as GKAP in older literature) has been shown to directly improve NMDA receptor activity [88]. Although little is known about SAPAP function in general, there are no studies that examine their relationship to drug reinforcement. This link is tantalizing considering that these proteins are linked to NMDA receptors, compulsive symptoms can be induced in rodents by deletion of the SAPAP3 isoform, and these effects can be reversed by traditional treatments for compulsivity [89]. In light of the success of trazadone in ameliorating compulsive symptoms of cocaine users, exploring the role of these proteins in response to cocaine sensitization may lead to targeted therapies for treating cocaine dependence.

Towards the goal of improving the knowledge base of how drugs of abuse create addicts and using currently uninvestigated areas of this knowledge base, we focused our research studies to the male albino rat, and attempt to uncover how cocaine sensitization alters cocaine self-administration, prefrontal glutamate receptors, and mesocorticolimbic SAPAPs. Even though there is a wealth of data on the effects of cocaine-sensitization on self-administration, few address the effects of sensitization after a period of abstinence 
using a dose high enough to elicit a response in sensitized animals, yet low enough to avoid repeat exposure causing sensitization. We hypothesize that using a consistent lowdose, cocaine self-administration will yield a significant elevation in drug-seeking behavior for psychostimulant sensitized animals. While the population and function of iGluRs are known to be altered throughout the mesocorticolimbic system, this effect has not been explored in the prefrontal cortex. The prefrontal cortex warrants this investigation because of sensitization-induced prefrontal glutamatergic pathology. Also absent in the literature are molecular links to observed clinical signs of compulsive behavior that can manifest in cocaine addicts, including unwanted obsessive thoughts about drugs, ritualistic/repetitive behaviors [90], and compulsive foraging for additional, non-existent drug [17]. Although it has been noted in the literature that rodents can be induced to display compulsive behaviors by deletion of synaptic SAPAP3 protein, any link bridging cocaine sensitization to the SAPAP family of proteins is wholly unexplored. For cocaine-induced molecular changes, we hypothesize that acute cocaine exposure and/or cocaine sensitization will alter iGluRs levels in the mPFC, and SAPAP levels in multiple sites of the mesocorticolimbic system.

To test these hypotheses, we will investigate the effects of psychostimulant sensitization on various parameters of self-administration by infusing a low-dose cocaine reward proven to not induce sensitization during self-administration (reported here as 0.3 $\mathrm{mg} / \mathrm{kg} /$ infusion). We will also investigate the effects of acute cocaine exposure and/or cocaine sensitization on iGluRs in the mPFC and SAPAPs in the entire mesocorticolimbic circuit through western blotting, immunolabeling of proteins of interest, and comparing protein levels to those found in cocaine naïve controls. A limited self-administration protocol will also test if SAPAP levels are altered by selfadministration of a low-dose cocaine reward.

Although the personal accounts related to cocaine use are as varied as there are drug users in the world, three personal accounts may help to place into to focus the realworld consequences on people of a disease that is often modeled in a laboratory using rodents:

I. "My academic expertise about drugs was a hindrance to my recovery. I was convinced my knowledge of drugs would make them more manageable. I now realize that this is like a ballistics expert thinking he is bullet-proof. My arrogance and delusion would be funny if they hadn't come so close to doing me in."-Narcotics Anonymous (1987) [91, p. 288]

II. "The decision-making part of the brain of an individual who has been using crystal meth is very interesting. When Carly and Andy were in their apartment, they ran out of drugs. They sold every single thing they had except two things: a couch and a blow torch. They had to make a decision because something had to be sold to buy more drugs. A normal person would automatically think, Sell the blow torch. But Andy and Carly sat on the couch, looking at the couch and looking at the blow torch, and the choice brought intense confusion. The couch? The blow torch? I mean, we may not need the blow torch today, but what about tomorrow? If we sell 
the couch, we can still sit wherever we want. But the blow torch? A blow torch is a very specific item. If you're doing a project and you need a blow torch, you can't substitute something else for it. You would have to have a blow torch, right? In the end, they sold the couch."-Dina Kucera (2010), Everything I Never Wanted to Be: A Memoir of Alcoholism and Addiction, Faith and Family, Hope and Humor [92, p. 165]

III. "Crack [Cocaine] is a motherfucker......the cocaine isn't the problem, it's the money [that one spends on it]. As soon as you get a dollar, it's the first thing you want [to buy]."-Personal communication from Kevin Brown, former cocaine addict, on March 15 ${ }^{\text {th }}, 2014$ 


\section{CHAPTER 2. EFFECTS OF SENSITIZATION ON COCAINE SELF- ADMINISTRATION}

\section{Rationale}

Cocaine sensitization causes increased drug-induced locomotion, and is also thought to increase the reinforcement potential of cocaine under operant training. Although there are studies that examine the effects of cocaine sensitization on low-dose cocaine self-administration (defined as approx $0.3 \mathrm{mg} / \mathrm{kg}$ ), there are factors that prevent adequate interpretation of the effects of sensitization on self-administration motivation and acquisition. Here, we used a between-sessions model of a low-dose in all selfadministration phases to measure the effects of sensitization on self-administration. After acquisition of self-administration, we tested for motivation, extinction, and reinstatement on different days to avoid acute cocaine-induced escalation and unwanted sensitization during self-administration. Using this low-dose protocol, significantly more sensitized animals completed the training protocol under this lower dose when compared to saline controls. Differences were not observed for days to complete the training protocol, reinforced responses under a progressive ratio $(\mathrm{PR})$ schedule, or active responses during extinction. Differences were also not observed for reinstated responses after injections of cocaine or yohimbine, a facilitator of reinstatement in cocaine sensitized rats. These results suggest that under a consistent sub-sensitizing dose, cocaine sensitization had a profound effect on the acquisition of cocaine self-administration behavior. Despite differences in acquisition, low-dose self-administration in sensitized animals did not result in escalation of drug seeking, or stress/drug primed reinstatement.

\section{Background}

Cocaine sensitization has long been hypothesized to contribute to the persistence of cocaine abuse in addicts. In rats, sensitization is defined as the ability of intermittent and repetitive doses of cocaine to cause long-term neuronal modification, and elicit elevated drug responses. These modifications are manifested behaviorally, and include enhanced drug-induced locomotion, seemingly useless repetitive movements, and enhanced acquisition of reinforced responding for cocaine under operant testing. Drug self-administration has long been used to examine the reinforcement potential of cocaine, and mimics various facets of drug abuse observed in human addicts, including the reinforcement of drug concentrations aversive to drug naïve controls, higher break point during progressive ratio (PR) testing, and continued reinforcement despite negative consequences. Self-administration studies have become an invaluable tool used by researchers to study how molecular and behavioral manipulations directly affect the reinforcement potential of cocaine. Yet, fewer of these studies examine how cocaine sensitization influences self-administration. Among the studies that do examine the effects of cocaine sensitization, there are factors that prevent interpretation of the effects of sensitization alone on self-administration motivation and acquisition. 
In examining all the variations in protocol, there are combinations of treatments that would alter any resulting cocaine reinforcement caused by cocaine sensitization. Drugs of abuse share the common feature of increasing dopamine in the nucleus accumbens (Nacc). Yet, the mechanism by which this occurs may vary, along with systemic effects and drug-induced neuroadaptations [93]. Rats sensitized to amphetamine display behavioral cross-sensitization to the reinforcing properties of cocaine [94], enhancement of cocaine seeking by injections of AMPA into the Nacc [95], and increased escalation of cocaine self-administration over non-sensitized controls [96]. Yet, amphetamine works through a different mechanism than cocaine to increase synaptic dopamine [97] which results in more severe dendritic changes when compared to cocaine exposed animals [76]. When cocaine and alcohol are combined, the resulting metabolites combine to produce cocaethylene [98]. Cocaethylene is longer lasting than both parent substances, more toxic than cocaine alone [99], and has a higher binding affinity for the dopamine transporter [100]. Due to these traits, cocaethylene not only masks, but supersedes any behavioral effects due solely to cocaine. Additionally, chronic stress causes alterations in the HPA axis that are linked with long term cocaine use and modification of reinforcement responses for cocaine (for detailed reviews see [101] \& [102]). This 'priming' by chronic stress could interfere with any examination of cocaine effects by chronic stress mimicking cocaine through increasing Nacc dopamine [103]. Stress priming in adolescent animals can sensitize drug naïve animals to cocaine [104], and may even render animals vulnerable to relapse [105]. In the study that compared two different doses during the course of the experiments, it was reported that all rats treated readily learned to self-administer, yet an earlier dose $(0.2 \mathrm{mg} / \mathrm{kg})$ was exchanged with a higher one $(0.8 \mathrm{mg} / \mathrm{kg})$ later in the protocol that is greater than one known to sensitize rats during the self-administration protocol [106]. This makes it unclear which dose is responsible for the reported effects, and risks sensitizing animals during testing. Another study used a non-sensitizing dose and a between-sessions model, but only required the animals to respond under a fixed ratio of one response (FR1) for 8 days [107]. Although this model spreads drug testing over separate days to avoid cocaine induced escalation/tolerance, this particular protocol may not have been stringent enough to establish differences between sensitized and non-sensitized animals.

While avoiding issues that muddle the interpretation of results, there are also several self-administration techniques that aid in the isolation of any effects caused by sensitization. Common in the literature examining the effects of cocaine sensitization on self-administration is a limitation on when drug is available to be infused. This limitation is included because animals given 24-hour free access to drug can quickly infuse enough drug to die from severe cocaine toxicity [108]. One author partially attributed this "loss of control" to cocaine-induced stereotypy [109]. In comparison, increasing the labor cost associated with drug infusions through the inclusion of a progressive ratio (PR) schedule has the ability to increase the reinforcement strength of cocaine self-administration (termed salience) by requiring seemingly random, ever-increasing effort to acquire subsequent doses of drug until a breakpoint is reached. At this breakpoint, the animal is unwilling to continue responding for the next dose, or cannot reach criteria in the allotted time $[110,111]$. To accomplish this, PR testing uses a specific formula to generate the number of responses needed to achieve the next dose and increases in a dynamic fashion, 
in contrast to a discrete number of responses under FR [111]. Analysis of breakpoints is critical because they serve as a more direct measure of reinforcement efficacy than FR responses that increases alongside motivation for drug-seeking [112]. Moreover, the efficacy of timeouts and PR testing can be affected by the time course over which the entire self-administration experiment takes place, and the length of each session. Although desirable for testing the effects of "binge" cocaine exposure, a within-session model is maladapted to studies measuring the effectiveness of sensitization. This is because prolonged daily cocaine exposure has been reported to either artificially increase drug reinforcement [113], or induce short-term drug tolerance [114]. Because of this fact, studies designed to test acquisition and maintenance of cocaine responding can be hindered by within-session models. For this reason, using a between-sessions model is more desirable.

Other than altering the operant protocols for self-administration, perhaps the simplest technique is to alter the concentration of each cocaine infusion ("dose") to be administered. The drug concentration is of crucial importance, and care must be taken in consideration of the self-administration dose. For example, although higher doses increase responses for drug, it may also be noxious to test animals depending on exposure history [115]. Yet, it is desirable to use a dose high enough to be reinforcing, as a dose that is too low will not elicit responding regardless of past history of cocaine exposure [116].

Operant testing for self-administration often involves several distinct phases. First, after implantation of an indwelling intravenous catheter, animals must be trained to associate operant responding with drug availability, a phase known as acquisition (or "training"). Second, after animals have met the criteria for training, the main testing phase follows in which different facets of operant responding are tested. This can range from responding under various schedules, to extinction of drug responding, to possible reinstatement of the responding behavior. Regardless of the findings that cocaine selfadministration prolonged over days can result in either elevated responses due to cocaineinduced enhanced locomotion or decreased responses due to acute tolerance-like effects, intermittent exposure to cocaine during self-administration over multiple days regardless of session length, results in an increase in reinforcement potential [117] and cocaine sensitization [106]. Unfortunately, there has been a noticeable dearth of studies specifically examining this facet of the addiction process in drug addicts [14], although a limited studies in non-drug users injected with amphetamine over 4 days report an increase in spontaneous blink rate and euphoria $[15,16]$.

In this manuscript, we used various methods to limit cocaine exposure to determine if cocaine sensitization affects acquisition of cocaine self-administration. These experiments will use a dose reported to be low enough to avoid cocaine-induced hyperactivity/ tolerance (defined here as $0.3 \mathrm{mg} / \mathrm{kg}$ ) to isolate any effects of cocaine sensitization, and to examine any altered responding under acquisition (training), PR schedule, extinction, and reinstatement of drug self-administration with challenge dose of cocaine $(15 \mathrm{mg} / \mathrm{kg})$. We used amphetamine pretreatment and yohimbine induced reinstatement as experimental controls. As justification, amphetamine pretreated rats are 
not only cross-sensitized to cocaine [94], but display enhanced escalation in cocaine selfadministration over non sensitized controls [96]. Also, the ability of yohimbine, an alkaloid that elicits reinstatement of extinguished cocaine self-administration [118], was used in reinstatement testing to evaluate the efficacy of cocaine exposure during reinstatement.

\section{Methods}

\section{Sensitization}

68 male Sprague-Dawley rats (Harlan Laboratories), weighing $250 \mathrm{~g}$ upon arrival were placed on a reverse daylight cycle (light: 1900hrs-0600/ dark: 0600-1900) and allowed to acclimate to for 5-7 days. Animals were allowed food and water ad libitum. After the acclimation period, subjects received intraperitoneal (I.P.) injections of cocaine, amphetamine, or saline only in identical volumes. Animals received an IP injection of 15 $\mathrm{mg} / \mathrm{kg}$ cocaine, 1.5 of $\mathrm{mg} / \mathrm{kg}$ of d-amphetamine, or saline on Day 1 and then every third day until a total of 5 injections had been administered. On the day following the last injection, sensitized animals were tested alongside saline-injected control rats for enhanced drug induced locomotion in infrared home cage monitors (Accuscan Instruments Inc.) with 16 infrared beams spaced approximately 0.8 inches apart. After a 1 -hour baseline, rats from all groups received a $15 \mathrm{mg} / \mathrm{kg}$ I.P. challenge dose of cocaine, and were placed back into the test chamber for an additional $2 \mathrm{hr}$ to record cocaineinduced activity.

\section{Jugular Catheter Implantation}

In the 24-72 hours following the locomotion test, rats were anesthetized with ketamine/xylazine cocktail (80 and $10 \mathrm{mg} / \mathrm{kg}$, respectively) and implanted with an indwelling silastic unilateral jugular catheter [119]. I.P. injections of $0.5 \mathrm{mg} / \mathrm{kg}$ Rimadyl ${ }^{\circledR}$ (Carprofen, injectable) for pain control and catheter injected $0.1 \mathrm{ml}$ of Baytril ${ }^{\circledR}$ (Bayer Corporation) were given immediately after surgery. Rats were allowed to recover for at least 5 days before testing. Catheters were flushed with $0.1 \mathrm{ml}$ of Baytril ${ }^{\circledR}$ daily for the first 7 days after implantation, and once every 7 days following until the conclusion of self-administration testing.

\section{Self-Administration Training}

Nine days following catheter implantation, rats were tested for catheter patency by experimenter-administered perfusions of $0.1 \mathrm{ml}$ fast acting/clearing Brevital ${ }^{\circledR}$ (Methohexital Sodium) through the catheter. If the catheter was unobstructed, the perfusion immediately anaesthetized the animal for approximately 1 minute. If animals were not anaesthetized, we concluded that the catheter was obstructed and the animals 
were immediately humanely killed by carbon dioxide asphyxiation. One day following the Brevital® patency test (Ten days after the last IP sensitization injection), rats were placed in operant behavior chambers (Med Associates Inc.) connected to a delivery system that delivered a 20 -second $100 \mu \mathrm{l}$ infusion of $0.3 \mathrm{mg} / \mathrm{kg}$ cocaine solution. The delivery system used operated at 3.33 revolutions per minute (RPM), and was capable of infusing $1.0 \mathrm{~mL} / \mathrm{minute}$. The chamber contained two active levers located side-by-side on the front "wall", a circular cue light on the wall over each lever, and a house light on the back wall opposite the levers. At the start of each session with the house light off, the cue light over the active lever was activated for 20 seconds. The cue light was then turned off, the house light turned on, and the "active" and "inactive" levers presented. When pressed, the active lever activated the delivery pump to infuse cocaine, activated a cue light over the lever, and turned out the house light. Pressing the inactive lever had no programmed consequences except event recording. To successfully complete the acquisition phase, animals were required to make nine infusions within 3 hours under the FR1 and FR2 schedules. Rats were first trained to self-administer cocaine under an FR1 schedule, during which every response on the correct lever resulted in a 20 -second $100 \mu 1$ infusion of cocaine for up to 9 responses within $3 \mathrm{hr}$. Upon successful completion of the FR1 schedule rats were challenged under an FR2 schedule, during which every second response resulted in an infusion, for up to 9 cocaine infusions. Rats were given 5 days to complete both the FR1 and FR2 schedules, unless the FR1 training was completed on the 5 th day. In this case, the rats were given one extra day to complete FR2. Any animals that did not meet these criteria were humanely euthanized by carbon dioxide asphyxiation in accordance with UTHSC IACUC protocols. After each session, behavioral chambers were thoroughly cleaned with $70 \%$ ethanol and stocked with fresh sanitized woodchip bedding.

\section{Self-Administration Testing}

On the day following completion of training, subjects were then required to respond for cocaine under a PR schedule [111] for 4 days. If the subject reached 30 infusions, the session was terminated before the three-hour time limit. After the conclusion of PR training on the same day, catheter patency was tested by perfusion of $0.1 \mathrm{ml}$ of Brevital $\AA$. The following day, subjects were placed on an extinction protocol for 3 days, during which cocaine was replaced by cocaine on the PR schedule. This extinction protocol also inactivated the cue light when the active lever was pressed. Following extinction training, the ability to re-instate responding was tested using a modified PR protocol in conjunction with extinction. Using this method, half of the animals were tested under 'reinstatement 1' conditions on the first day in which $15 \mathrm{mg} / \mathrm{kg}$ cocaine or $2 \mathrm{mg} / \mathrm{kg}$ yohimbine was injected IP. On days 2-4, the extinction protocol described above was repeated. On day 5, rats were tested under similar conditions as the first reinstatement day except an injection of saline was administered. The remaining half of animals were tested under similar conditions except saline was delivered after the first extinction sessions and yohimbine/cocaine was delivered after the second extinction

sessions. At the conclusion of self-administration, rats were euthanized by carbon dioxide asphyxiation in accordance with UTHSC IACUC protocols. 


\section{Analysis}

All statistical calculations were performed using Prism 5 (Graphpad). Analysis consisted of a percentage of total animals of each treatment group that completed training was analyzed by a Chi-Square analysis for trends, and series of two-way ANOVA tests for analyzing the post-sensitization cocaine challenge dose, sensitized animals for PR testing, Extinction phase 1, Reinstatement phase 1, Extinction phase 2, and Reinstatement phase 2.

\section{Results}

Verified by challenge injections of cocaine, all animals subjected to the sensitization protocol were sensitized by cocaine exposure, or proven to be crosssensitized to cocaine by amphetamine exposure $(\mathrm{F}=9.319(2,33), \mathrm{p}=0.0003$, Figure 2-1). Significantly higher photocell counts over controls were displayed by cocaine pretreated and amphetamine pretreated animals. On the following day, the self-administration experiments commenced. Although animals did not show any blockages in the intravenous catheter or signs of surgery related infection or discomfort, many of the saline control animals failed to complete the training protocol in the allotted 5-day time period compared to the drug-treated controls (Figure 2-2). A chi square analysis for trends revealed a difference between untreated and treated animals for the numbers of animals that completed the training protocol $(x 2(1)=4.669, p=0.0307$, Figure 2-3). Among the animals that did complete the training protocol, the self-administration protocol continued as outlined, although with a ratio skewed towards a greater representation of drug treated animals. Using a two way ANOVA, no differences between treatment groups were observed during the PR schedule, extinction schedule, or yohimbine/cocaine induced reinstatement. For these schedules, analyzed behaviors included active lever responses, inactive lever responses, infusions earned, and session duration.

\section{Discussion}

Although not significant for most cases, several interesting trends emerge among these data. Firstly, the failure of saline controls to complete training is a surprisingly novel result. It is known that the dose response curve of sensitized animals is shifted, which would explain the result of drug-sensitized animals completing the training with more frequency over control animals. Yet, upon further examination of data, it was also noted that for saline controls that did not complete training, not only did the animal not meet criteria over the initially allotted five days, but often stopped responding completely after the first day. This would suggest that this $0.3 \mathrm{mg} / \mathrm{kg} /$ infusion dose was not reinforcing to control animals.

The technique used to deliver drug to the animal could have affected the selfadministration results in our study. In fact, the published study that our self- 


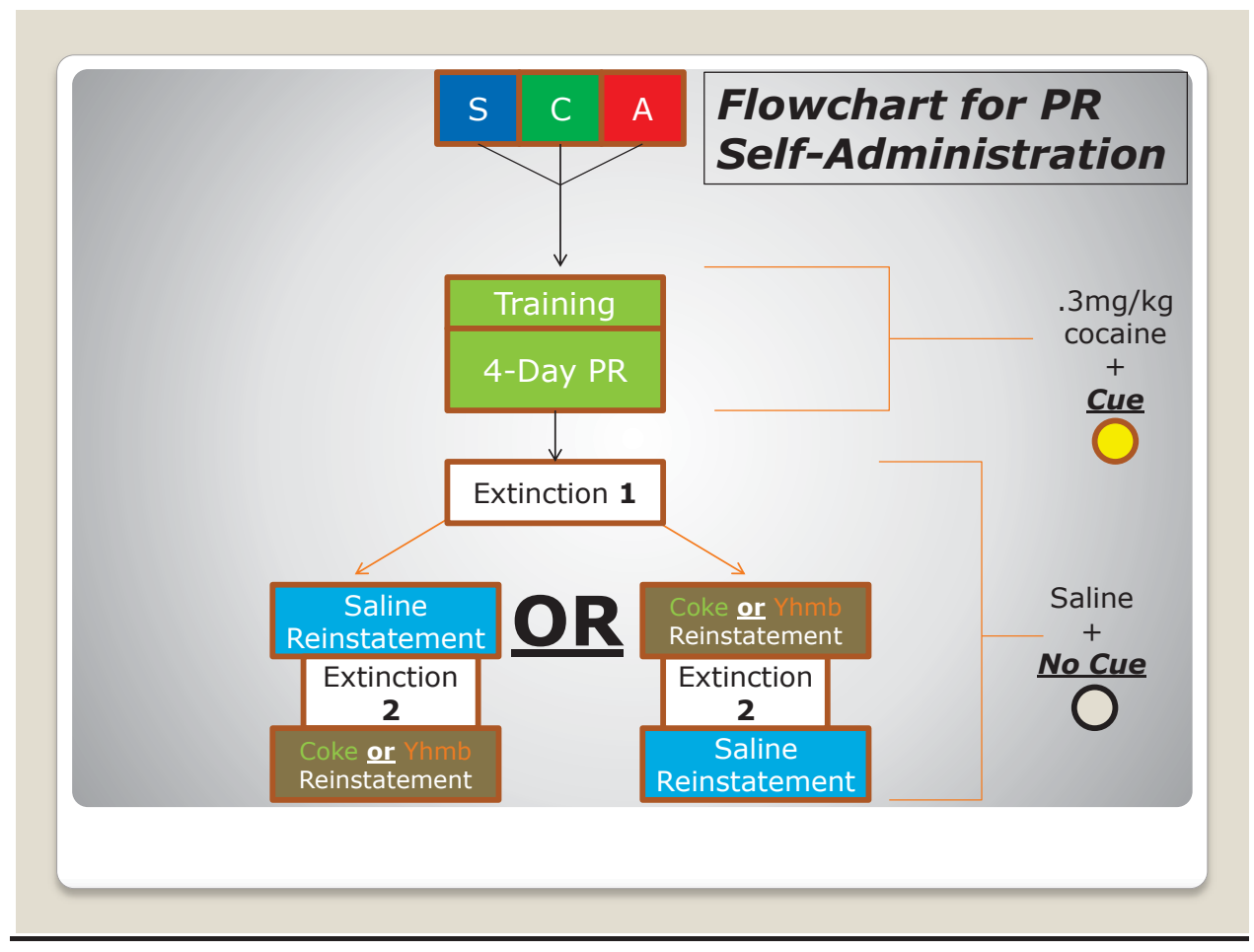

Figure 2-1. Graphic Flowchart for Self-Administration Protocol.

Animals segregated by pretreatment are indicated at the top middle: the blue box containing " $\mathrm{S}$ " represents control animals, the green box containing " $\mathrm{C}$ " represents cocaine sensitized animals, and the red box containing " $\mathrm{A}$ " represents amphetamine sensitized animals. The animals where then tested using the same paradigm, and under reinstatement animals were randomly separated into two counterbalanced groups. 


\section{Cocaine Challenge}

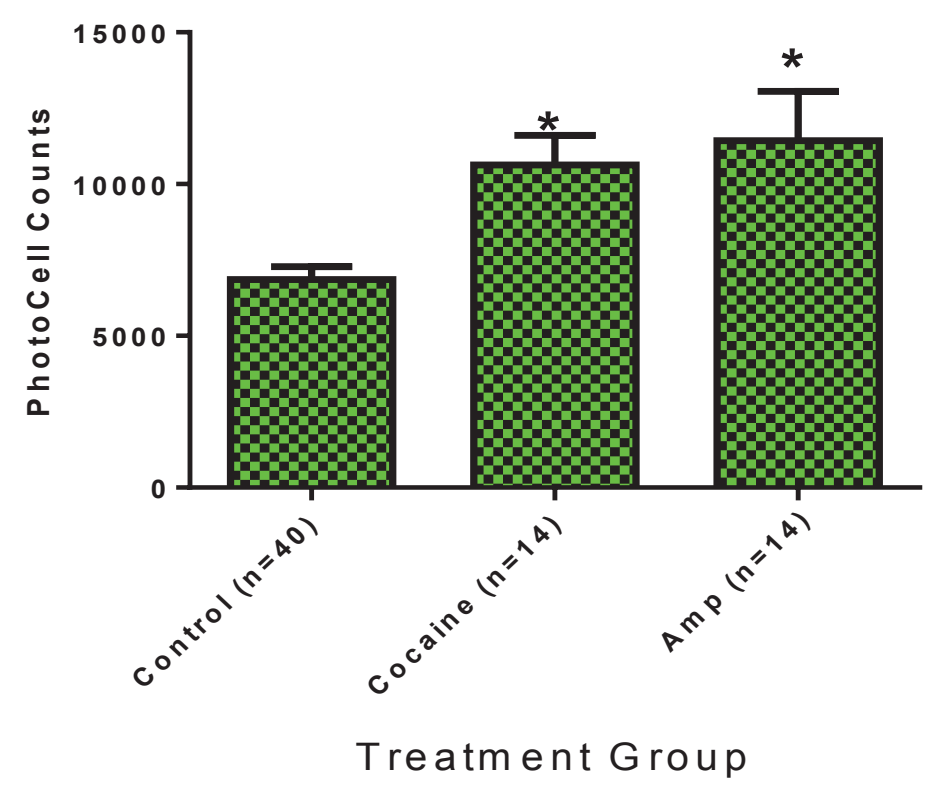

Figure 2-2. Sensitization Verification by Infrared Home Cage Monitors.

Testing included a 1-hour activity baseline, followed by a 2-hour activity recording that was immediately preceded by a challenge dose of $15 \mathrm{mg} / \mathrm{kg}$ cocaine I.P. injection.

Photocell counts for were significantly higher in cocaine challenged cocaine and amphetamine (Amp) sensitized animals, indicated by “*”. 


\section{Chi Square Analysis \\ of Acquisition}

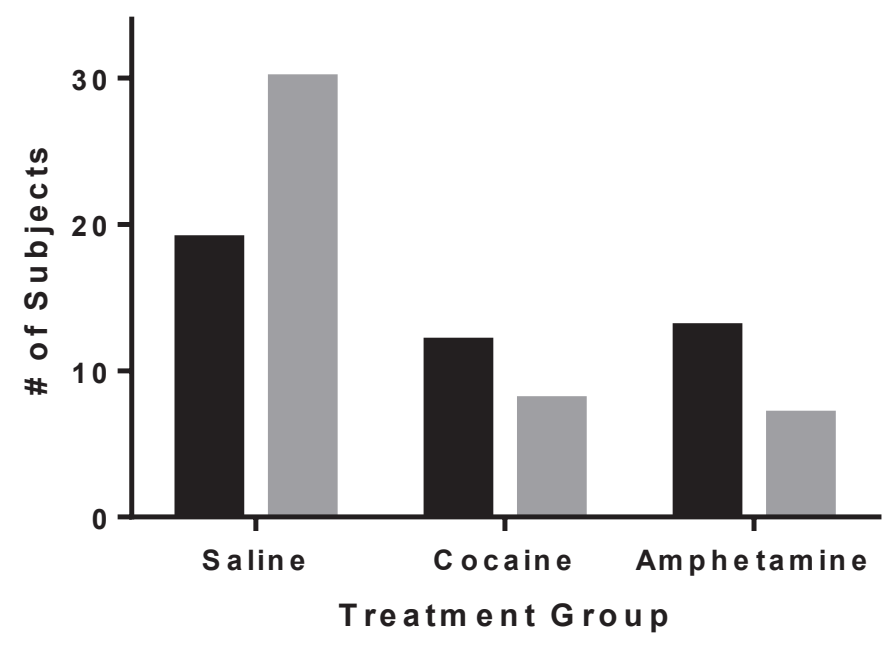

Passed

Failed

Figure 2-3. Chi Square Analysis Comparing Treated and Untreated Animals During Acquisition.

Under Chi-Square analysis, there is a trend difference between treated and untreated animals acquiring self-administration behavior during the FR1/2 self-administration training protocol. Notice that more untreated (saline) animals were tested, and a significantly greater proportion of saline animals fail to acquire the behavior. 
administration experiments were based on used faster pumps that delivered $1.6 \mathrm{ml} / \mathrm{minute}$ [95], compared to ours that infuse drug at approximately $1.0 \mathrm{ml} / \mathrm{minute}$. The relatively slow speed of the self-administration pumps could have lowered the efficacy of the cocaine delivery system, as faster speeds of delivery results in increased motivation during self-administration in rats $[120,121]$ and humans $[122,123]$. This explains why all routes of cocaine absorption used by humans facilitate concentrated, rapid delivery of concentrated drug doses into the bloodstream [124, 125]. Indeed, without a concurrent increase in delivery speed, independent studies report that under a significantly higher dose $(0.8 \mathrm{mg} / \mathrm{kg})$, not only did most animals complete the training phase but also an increase in cocaine self-administration under PR was significantly increased.

Additionally, the study these experiments were based on precipitated reinstatement by intracranial injection of $\alpha$-Amino-3-hydroxy-5-methyl-4-isoxazolepropionic acid (AMPA), an agonist that provides greater stimulation properties by displaying 2-3 times the affinity for AMPA-type glutamate receptors than the endogenous glutamate present in our sensitized animals [126]. Second, animals were sensitized in a different environment than the operate chamber. Treating subjects in the same environment as the one used for operate testing is known to increase the locomotor effects of cocaine sensitization [127, 128]. The level of sensitization in our sensitized subjects may not have been sufficient to alter self-administration over cocaine naïve controls.

In conclusion, the significant increased number of sensitized animals acquiring self-administration at this dose is a novel finding. These results may suggest that animals not vulnerable in some way to the reinforcing effects of cocaine will find much lower doses of drug than previously thought reinforcing, and cocaine naïve controls are much more resistant than previously believed to acquiring cocaine self-administration. 


\section{CHAPTER 3. EFFECTS OF COCAINE SENSITIZATION ON SAPAP LEVELS IN THE MESOCORTICOLIMBIC SYSTEM}

\section{Rationale}

In humans, symptoms associated with cocaine use are reported to include obsessive components including compulsive foraging for drug and unwanted/intrusive thoughts of drug use, even among abstinent users. This obsessive feature of sensitization may be especially instrumental in relapse, and is associated with abnormal glutamate transmission in the mesocorticolimbic system. For various regions of the mesocorticolimbic system, we examined the effects of cocaine sensitization and acute cocaine exposure on SAPAPs. Rats were sensitized to cocaine over 4 days (15 $\mathrm{mg} / \mathrm{kg} /$ day) and along with matched sham-injected controls, were either exposed to an acute dose or saline injection after 1 day, 2 weeks, or 3 weeks of cocaine abstinence. We observed that cocaine sensitization results in significantly lower SAPAP protein levels in the dorsal medial prefrontal cortex (mPFC) and the nucleus accumbens (Nacc). In the nucleus accumbens core, a significant reduction in SAPAPs was only observed for sensitized animals exposed to drug on test day. In the dorsal prefrontal cortex, effects were less dependent on sensitization, and a reduction was observed in cocaine naïve controls and sensitized rats. This occurred in response to both acute exposures on test day and prolonged abstinence.

In a separate self-administration study, cocaine naïve rats were implanted with indwelling jugular catheter and lever pressed for a cocaine reinforcer $(0.3$ $\mathrm{mg} / \mathrm{kg} /$ infusion) under FR1 schedule for 10 days, yoked to animals that received saline infusions simultaneously. In test animals, increased SAPAP protein levels were observed for all 4 SAPAPs in the dorsal mPFC, SAPAP3 in the ventral mPFC, and SAPAP 2 and 4 in the nucleus accumbens core. In addition, SAPAP levels in the mesocorticolimbic circuit displays multiple significant alterations upon acute exposure at 2-24 hrs post exposure. Exploration of how these proteins are altered in sensitized animals and by contingently-administered cocaine may serve in identifying potential pharmacological treatments for drugs of abuse.

\section{Background}

Following the growing appreciation of glutamate in the addiction process, there has been a growing interest in glutamate receptors (GluRs) and how their physiology is altered by cocaine sensitization. These receptors are both ionotropic (iGluRs) and metabotropic (mGluRs), and work together to affect glutamate transmission. Metabotropic GluRs modulate cell responses indirectly through G-protein cascades, and alter the responses to glutamate by preventing hyperpolarization [129] and presynaptic silencing [130]. Despite the global influence of mGluRs, the direct and immediate effects of glutamate are mediated by pore-containing iGluRs that directly contribute to the postsynaptic current. The subunits composing iGluRs determine receptor function, and also 
display a limited ability to regulate mGluR activity [131]. Postsynaptically, these receptors consist in three distinct varieties: excitatory receptors that are activated by AMPA ( $\alpha$-Amino-3-hydroxy-5-methyl-4-isoxazolepropionic acid), receptors sensitive to NMDA (N-Methyl-D-aspartate), and a less well understood class of kainite-sensitive receptors [54]. With the exception of the prefrontal cortex, the details of how mesocorticolimbic AMPA/NMDA receptors are affected in number and function by cocaine exposure are documented, and trend towards a greater contribution of excitatory post-synaptic currents [132]. In schizophrenia and cocaine sensitization, increased AMPA receptor input and elevated dendrite number are evidence of enhanced glutamate sensitivity [133].

Although these receptors function individually, the physical clustering of multiple receptors facilitates efficient neurotransmitter responses [84]. These multi-receptor complexes are anchored to the surface of the PSD, and are associated with an elaborate transduction array below the cell surface that is connected to the neuronal cytoskeleton [84]. This array also anchors and condenses iGluR transduction complexes. This is an important function because mechanisms affecting the expression of cocaine sensitization include iGluR adaptations and intracellular signaling cascades. Similar to mGluRs, iGluRs have the ability to initiate cell signaling through their intracellular domains to regulate synaptic responses. NMDA receptors regulate the surface expression of AMPA receptors through the Ras-ERK pathway by NR2A-containing NMDARs promoting AMPAR expression, and NR2B subunits depressing this expression [72]. AMPA receptors even have the ability to remove themselves from the synapse via the GluR2 subunits through BRAG2-mediated endocytosis [73]. However, these signaling mechanisms may not only regulate the functional ratio of AMPA/NMDA receptors, but also alter the activity of receptors present at the synapse. For example, phosphorylation of the GluR1 AMPA subunit by PKC at Ser(831) increases AMPA channel conductance [74]. In addition to these phenomena, cytoskeleton remodeling is known to facilitate drug-induced habit formation in an unknown fashion [75].

In cocaine sensitized rats, clear and dramatic morphological changes occur in MSNs of the nucleus accumbens (Nacc) [76]. Splice variants of synapse-associated protein 102 (SAP102) are implicated in synaptic remodeling by subunit-specific regulation of NMDARs, anchoring these receptors to the synapse [77]. Following this finding, although there is no cure for addiction, NMDA receptors may be particularly important in ameliorating certain symptoms associated with addiction. Interestingly, trazodone, an NMDAR inhibitor and traditional medication for the treatment for obsessive compulsive disorder (OCD), has been used to successfully reduce compulsive drug-foraging in cocaine addicts. Yet, exactly how trazodone exerts this effect is unknown $[78,79]$. Although an unorthodox treatment for cocaine addiction, closer examination shows that several similarities are shared by cocaine addicts and OCD patients. These similarities include unwanted/intrusive anxiety-related thoughts and compulsive and repetitive behavior [80]. In fact, co-morbid diagnoses of both disorders are not uncommon [81]. Yet while trazodone inhibits the activity of ionotropic NMDA receptors, these receptors are linked at the PSD to the underlying cytoskeleton by SAP90/PSD-95 which recruits others proteins to the synapse [84]. One of the families of 
proteins that are recruited the receptor/SAP90 complex are SAP90-associated proteins, the commonly used acronym for which is SAPAP [134]. While SAP90 has been exhaustively studied ([84], review), relatively little is known about its associated SAPAPs. These proteins may be important proteins in the progression of the compulsive symptoms of cocaine addiction. Four isoforms of SAPAPs exist (SAPAP 1, 2, 3, and 4) and range in size from $90 \mathrm{kDa}-120 \mathrm{kDa}$ [85]. These proteins serve as the intermediary between SAP90 at the PSD and the underlying cytoskeleton [86]. These proteins also contain a conserved guanylate kinase domain that has been shown to activate NMDA receptors [87]. Specifically, SAPAP2 has been shown to directly improve reduced NMDA receptor activity [88]. Although little is known about how SAPAPs generally function, and nothing of their relation to drug reinforcement, their exploration in this role is tantalizing considering that these proteins are linked to NMDA receptors. This argument is bolstered by the finding that inhibiting GKAP degradation in the hippocampus results in a phenotype strikingly similar to Nacc MSNs due to cocaine sensitization [135]. In light of the success of trazodone in ameliorating the compulsive symptoms of cocaine users, exploring the role of these proteins in response to cocaine sensitization may lead to targeted therapies for treating cocaine dependence.

While their exact functions in addition to receptor clustering are not known, SAPAPs (also called DAP, or GKAP in older literature) also interacts with dynein in spindle formation [136], can recruit nNOS to the post synaptic density [137], and can be removed from the synaptic density due to increased excitatory activity [138]. SAPAPs also seem to be localized exclusively with cholinergic/glutamatergic synapses, and show differential expression spatially, with SAPAP3 being restricted to the dendrite and all others to the soma [139]. In mice, deletion of SAPAP3 in the striatum results in synaptic disorganization, excess grooming to the point of skin lesioning, altered corticostriatal transmission, and compulsive anxiety-associated behaviors [89]. In these animals, the resulting compulsive characteristics can also be rescued by viral transfection of full length SAPAP3 transcripts or treatment with fluoxetine, a drug treatment for OCD [89]. In humans, rare missense mutations in SAPAP3 are even implicated in patients suffering from the OC-spectrum disorder tricholliomania (compulsive hair eating) [140], giving clinical significance to information gained from model organism studies.

In addition to ionotropic iGluRs, SAPAPs may also influence mGluRs. According to one study, SAPAP3 deletion can cause striatal mGluR5-dependent silencing of AMPA receptors in addition to compulsive grooming [141]. Additionally, the mGluR5 agonist DHPG in the mPFC is sufficient to induce cross-sensitization to cocaine, and antagonist MTEP sufficient to prevent it [142], yet any links to SAPAPs are absent. To understand how SAPAPs may affect mGluR function, an understanding of the role of mGlur5 in the regulation of glutamate transmission is required.

SAPAP disruption may help to describe mGluR5 silencing, by considering the influence of Homer on mGluRs. The Homer scaffolding protein family regulates prefrontal glutamate transmission by clustering mGluR5 receptors, which is a process that is dependent on an association between Homer and a protein complex including GKAP (SAPAP1) [143]. Homer interacts with mGlur5 through its EHV1 domain to 
cluster together mGluR5 receptors, yet the Homerla isoform lacks the necessary cterminal domain to cross-link receptors and perform this function [143]. Lack of clustering of mGluR5 receptors, similar to NMDA receptors, would lower the efficiency of these receptors. As evidence of this phenomena, 10-days of cocaine self-administration in rats causes a long term elevation in Homerla/b in the ventral mPFC [144]. Cocaineprimed reinstatement can be extinguished in these animals by knockdown of Homerla in the ventral $\mathrm{mPFC}$, or increased expression of an isoform able to crosslink mGluRs, Homer1c [144]. Note also that increased stimulation concurrently increases Homer1a levels [145]. Considering the role of Homer in clustering mGlur5 receptors, this result may also be accompanied by increased excitatory glutamate transmission in the ventral $\mathrm{mPFC}$ by cocaine exposure.

When considering this result in the ventral $\mathrm{mPFC}$, recall that activating mGluR5 receptors in the $\mathrm{mPFC}$ can initiate behavioral sensitization, and inhibiting these receptors can prevent it. In addition, electrical stimulation enhances the locomotor effects of behavioral sensitization by stimulation of NMDA receptors ("kindling") [146], and activation also uncouples them from SAPAPs [138]. Even though these results are seemingly in conflict with one another, they are evidence that the dorsal and ventral $\mathrm{mPFC}$ have differing roles in the sensitization process that can be understood by examining how these regions act in concert with each other.

The dorsal mPFC is critical to the development of cocaine sensitization, and lesioning this area will prevent the initiation and expression of sensitization from occurring [147]. Conversely, administering the GABA agonist baclofen in the ventral mPFC will prevent acute cocaine-induced motor activity and initiation of sensitization, but not its expression [148]. Considering these studies together, a working model of changes in the dorsal and ventral $\mathrm{mPFC}$ in relation to cocaine sensitization can be assembled. In response to cocaine exposure, the dorsal $\mathrm{mPFC}$ is the seat of the initial $\mathrm{mPFC}$ changes of behavioral sensitization. Increased glutamatergic transmission in this region stimulates all glutamate receptors. This stimulation uncouples NMDA and mGluR5 receptors at the PSD, which lowers their efficiency. It also uncouples NMDA receptors from GKAP, and mGluR5 from complex-forming Homer proteins substituted with Homer1a. This would explain why mGluR5 agonists can cause sensitization and antagonists can inhibit sensitization, as antagonists can prevent receptor uncoupling. Additionally, if the dorsal $\mathrm{mPFC}$ is lesioned, this process is thwarted and behavioral sensitization cannot occur. If not prevented in some form, increased glutamatergic transmission will affect the ventral $\mathrm{mPFC}$ and produce similar uncoupling of inhibitory receptors. While GABA agonists can prevent the effects of enhanced glutamate transmission in the ventral $\mathrm{mPFC}$ caused by acute cocaine and the initiation of behavioral sensitization, they cannot reverse changes already present. This would explain why baclofen is ineffective in preventing the expression of behavioral sensitization. Reversing the effects of sensitization in the ventral mPFC can prevent behaviors associated with sensitization (i.e. substituting Homerla for Homer1c to prevent cocaine-induced reinstatement). Also note that all of the previously-attributed effects of cocaine exposure can also vary if cocaine is experimenter-administered (non-contingent) or if animals administer the drug to themselves (contingent). Considering that dopamine is 
preferentially increased more in the Nacc core than the shell in response to contingentlyadministered cocaine, it will be of value to determine how contingent cocaine exposure affects SAPAP levels.

Motivated by these published data, we propose here to investigate if behavioral sensitization and acute cocaine challenge are associated with changes of SAPAP protein isoforms in the ventral and dorsal $\mathrm{mPFC}$, Nacc core and shell, dorsal striatum, substantia nigra, and ventral tegmental area. In contrast to these studies relying upon non-contingent (experimenter-administered) drug, we will also investigate if and how SAPAP levels in the mesocorticolimbic circuit are altered by contingent (self-administered) cocaine exposure. This is an important detail to study considering the effects of cocaine can vary under these two treatment regimens even in non-sensitized animals acutely exposed to cocaine [149]. To further characterize how SAPAPs change in response to cocaine, we will also investigate the time course of any changes in response to acute cocaine exposure.

\title{
Methods
}

\section{Animal Subjects}

Upon delivery, male albino Sprague Dawley rats weighing approximately $250 \mathrm{~g}$ on arrival were acclimated to reversed light cycle housing (LIGHTS OFF - 05:00-18:00 hrs, LIGHTS ON- 18:00-05:00 hrs) for 3 days. Rats were housed individually and received food/water ad libitum for the duration of their lives.

\begin{abstract}
Abstinence Studies
Rats were sensitized by receiving an injection of cocaine $(15 \mathrm{mg} / \mathrm{kg})$ once a day at 12:00-13:00 for 4 days. A separate group of control animals received sham (saline) injections on the same schedule as sensitized test animals. Next, both groups of animals were divided into 3 different cohorts that underwent a period of abstinence for 21 days ( 3 weeks), 14 days ( 2 weeks), or 1 day. On the day following the abstinence period, sensitized animals were tested alongside time-matched and sham-injected control rats for any enhanced drug induced locomotion in infrared home cage monitors (Accuscan Instruments Inc.). A baseline measure of motor activity was obtained for $1 \mathrm{hr}$. Next, sensitized animals either were challenged with a dose of $15 \mathrm{mg} / \mathrm{kg}$ I.P. cocaine (cocaine/cocaine group) or received a sham injection of saline (cocaine/saline group). Cocaine naïve controls were examined alongside sensitized animals and either received a single injection of cocaine on test day (saline/cocaine group) or an injection of saline (saline/saline). Regardless of the injection administered, the challenge session was concluded after 2 hours. Immediately after the session, rats were sacrificed by guillotine without anesthesia.
\end{abstract}




\section{Acute Time Course Studies}

Acutely exposed animals were injected (I.P.) with $15 \mathrm{mg} / \mathrm{kg}$ cocaine and killed at either 2 hours, 4 hours, 8 hours, 12 hours, or 24 hours post-injection. Control animals were also killed at these same time points, and were sham-injected with saline. For methods detailing guillotine and tissue harvesting procedure, see "Tissue Harvesting" below.

\section{Jugular Catheter Implantation}

For self-administration animals, on the day of surgery subjects were anesthetized with ketamine/xylazine cocktail (80 mg/ $\mathrm{kg}$ and $10 \mathrm{mg} / \mathrm{kg}$, respectively) and implanted with a indwelling silastic unilateral jugular catheter [150], and given a I.P. injection of $0.5 \mathrm{mg} / \mathrm{kg}$ Rimadyl ${ }^{\circledR}$ (Carprofen, injectable) for pain control and catheter injected $0.1 \mathrm{ml}$ of Baytril ${ }^{\circledR}$ (Bayer Corporation) at the conclusion of surgery. Rats were then allowed to recover for at least 5 days before testing, and catheters were flushed with $0.1 \mathrm{ml}$ of Baytril® daily for the first 7 days after implantation, and once every 7 days following until the conclusion of self-administration testing.

\section{Self-Administration Training}

One day prior to self-administration testing, rats were tested for catheter patency by experimenter-administered perfusion of the $0.1 \mathrm{ml}$ of fast acting/clearing Brevital ${ }^{\circledR}$ (Methohexital Sodium). The day following the 5-day recovery period after surgery, rats were placed in operant behavior chambers (Med Associates Inc.) connected to delivery system that delivered a 10 -second $100 \mu \mathrm{l}$ infusion at 10 revolutions per minute (RPM). This system delivered a concentration of approximately $0.3 \mathrm{mg} / \mathrm{kg} /$ infusion of cocaine solution. Two levers were presented at the start of each session. These levers were an "active" lever (response activated the delivery pump to infuse cocaine, activated a cue light over the lever, and turned out the house light), and an "Inactive" lever (no programmed consequences for animals). Each animal actively infusing cocaine was "yoked", meaning its responses were replicated in a separate operant chamber containing a cocaine naïve control animal connected to an identical pump that administered saline, not cocaine. Yoked animals had no control over the actions of the levers or the lights. For the next 10 days from 1200 to $1500 \mathrm{hrs}$, rats were allowed to self-administer cocaine for $3 \mathrm{hrs}$ each day under fixed ratio 1 (FR1). After each session, behavioral chambers were cleaned with $70 \%$ ethanol and stocked with fresh sanitized woodchip bedding. Upon successful completion of the last session, rats were tested again for catheter patency. 3 weeks following the end of the last self-administration session, animals were sacrificed by guillotine without anesthesia. 


\section{Tissue Harvesting}

Tissue harvesting procedures were identical for all experiments. Immediately following guillotining, rat brains were removed intact from the skull and placed on ice. The brain was then dissected for the medial prefrontal cortex (mPFC, separate dorsal and ventral), nucleus accumbens (Nacc, separate core and shell regions), dorsal striatum (Str), substantia nigra (SN, combined pars reticulata/compacta), and ventral tegmental area (VTA) using established protocols [151]. Immediately after removal of each region, samples were placed on dry ice. From the time of guillotining to the sample incubation in dry ice, less than 3 min elapsed. For longer term storage, samples were stored at $-80^{\circ} \mathrm{C}$. All animal experiments were conducted in accordance with accordance with UTHSC IACUC protocols.

\section{Initial Preparation, Protein Assay, and Immunoblotting}

Whole tissue samples were sonicated in 2\% SDS and centrifuged at 13,000 X g for 30 secs. To obtain the protein concentration of the sample, $200 \mu 1$ of this whole tissue sample preparation was analyzed in triplicate using a Bio-Rad ${ }^{\circledR}$ DC ${ }^{\text {TM }}$ Protein Assay Kit. The triplicate readings were average together, and this average was used as the protein concentration. An aliquot of tissue sample was diluted to $2.0 \mu \mathrm{g}$ protein $/ \mu 1$ with $2 \% \mathrm{SDS}$. An aliquot of this diluent was diluted to $1 \mu \mathrm{g}$ protein/ $\mu \mathrm{l}$ with an equal volume of $2 \mathrm{x}$ electrophoresis sample buffer [final concentration after dilution $=50 \mathrm{mM}$ Tris $\mathrm{HCl}(\mathrm{pH}$ 6.7), 4\% glycerol (wt/vol), 2\% SDS, $5 \mathrm{mM}$ dithiothreitol and bromophenol blue (0.01 $\mathrm{mg} / \mathrm{ml})]$. Proteins $(20 \mu \mathrm{g} / \mathrm{lane})$ were separated by size on a $10 \% \mathrm{SDS}$-polyacrylamide gel transferred to Immobilon-P PVDF transfer membranes (Millipore) in Towbin's-SDS transfer buffer (25 mM Tris, $192 \mathrm{mM}$ glycine, 20\% methanol, and $0.01 \% \mathrm{SDS}$ ) at 100V, $0.35 \mathrm{~A}$ for 1-hr at room temperature with the transfer apparatus packed in ice. After transfer, the membrane was removed from the transfer apparatus and was left to dry overnight at room temperature.

Membranes were reactivated after 12-hrs using 1x 20-sec methanol wash, followed by 2 x 30-sec washes in ddH20, 1x 10-min wash in ddH20, and 1x 15-min wash in TBST. Membranes were then blocked in TBST containing 5\% nonfat dried milk (NFDM, Blotting Grade Blocker, Bio-Rad) for 1-hr at room temperature with agitation. The membrane was then washed once with TBST for 5-min and incubated with the primary antibody (SAPAP1,SAPAP2,SAPAP3,SAPAP4 at 1:12,500; Pro-Sci Inc., Poway, CA) diluted in TBST containing 0.5\% NFDM for 1-hr. As an experimental control, all throughout the labeling process, another portion of each corresponding membrane was concurrently probed with an anti-beta tubulin antibody (Sigma Aldrich). The membranes were then washed once for 15-min, and then twice more for 5-min in TBST followed by 1 -hr incubation with agitation at room temperature with horseradish peroxidase-conjugated goat anti-rabbit immunoglobulin G (Pro-Sci) diluted 1:5000 in TBST containing $0.5 \%$ NFDM. After this incubation, the membrane washing technique following primary antibody incubation was repeated, and the antigen-antibodyperoxidase complex was detected by enhanced chemiluminescence (West Femto 
Substrate, Thermo Scientific) according to the manufacturer's instructions and visualized, analyzed and quantified by computer-assisted densitometry using a Bio-Rad Molecular Imaging System using Quantity One Imaging Software. After visualizing, the membranes were then stripped using $0.1 \mathrm{M}$ glycine stripping buffer $(\mathrm{pH}=2.2)$ containing $1 \%(\mathrm{v} / \mathrm{v})$ Tween-20. After each stripping, the protocol was restarted at the steps mentioned above after methanol reactivation until all SAPAPs were probed.

\section{Analysis}

In analyzing western blots, each SAPAP/beta tubulin band in each sample lane was measured and using the imaging software, its adjusted percent volume was calculated, globally subtracting any background present. . Beta tubulin was chosen as an unchanging control due to the absence of literature reporting cocaine-induced changes in quantity, or post-translational modifications in contrast to alpha tubulin $[152,153]$. Each SAPAP adjusted percent volume value was then divided by its corresponding beta tubulin value using Microsoft Excel for each sample after every labeling with each primary antibody. For self-administration studies, data were collected for each test animal only, as the data for yoked controls was non-existent and all experiences were linked to the active responses of test animals. All statistical calculations were performed using Prism (Version 5, Graphpad Software Inc.), including SEM for all analysis, unpaired t-test for SAPAP in self administration test and yoked rats, and for examining acutely treated animals against their time matched sham injected controls. One-way ANOVA analysis (Newman-Keuls multiple comparison post-test) was used for all other analysis.

\section{Results}

For general trends, SAPAP levels in the mPFC decreased 2-8 hrs after acute cocaine exposure, and began to recover to baseline levels at $12 \mathrm{hrs}$ (Figure 3-1). In the dorsal $\mathrm{mPFC}$, when compared to time-matched cocaine naïve controls, several SAPAPs displayed a decrease at multiple time points. SAPAP1 was significantly reduced $(\mathrm{F}=$ $8.315(5,32), p=0.0001$, Figure 3-2A) at $2 \mathrm{hrs}, 4 \mathrm{hrs}, 8 \mathrm{hrs}$, and $24 \mathrm{hrs}$. SAPAP2 was significantly reduced $(\mathrm{F}=5.013(5,32), \mathrm{p}=0.0017$, Figure 3-2E) at $2 \mathrm{hrs}, 4 \mathrm{hrs}$, and 12 hrs after acute cocaine exposure. SAPAP3 was significantly reduced $(\mathrm{F}=8.630(5,32), \mathrm{p}=$ $<0.0001$, Figure 3-2C) at $8 \mathrm{hrs}$, and $24 \mathrm{hrs}$ after acute cocaine exposure. SAPAP4 was significantly reduced $(\mathrm{F}=5.249(5,32), \mathrm{p}=0.0012$, Figure 3-2G) at $2 \mathrm{hrs}, 8 \mathrm{hrs}$, and 24 hrs after acute cocaine exposure. SAPAPs were also decreased in a time-dependent manner in the ventral mPFC. SAPAP1 was significantly reduced in acutely exposed animals $(\mathrm{F}=7.278(5,32), \mathrm{p}=0.0001$, Figure 3-2B) at $2 \mathrm{hrs}, 4 \mathrm{hrs}, 8 \mathrm{hrs}$, and $24 \mathrm{hrs}$ after acute exposure. SAPAP2 was reduced $(F=3.697(5,32), p=0.0094$, Figure 3-2D) at 4 hrs and 8 hrs. SAPAP3 $(F=3.961(5,32), p=0.0066$, Figure 3-2F) and SAPAP4 $(F=4.104, p=0.0054$, Figure $3-2 H)$ were only significantly reduced at 8 hours after acute exposure. Significant changes were also observed at multiple time points for other regions examined (Table 3-1, $\mathrm{n}=4$ and $\mathrm{DFn}, \mathrm{DFd}=5,32$ for all groups). 

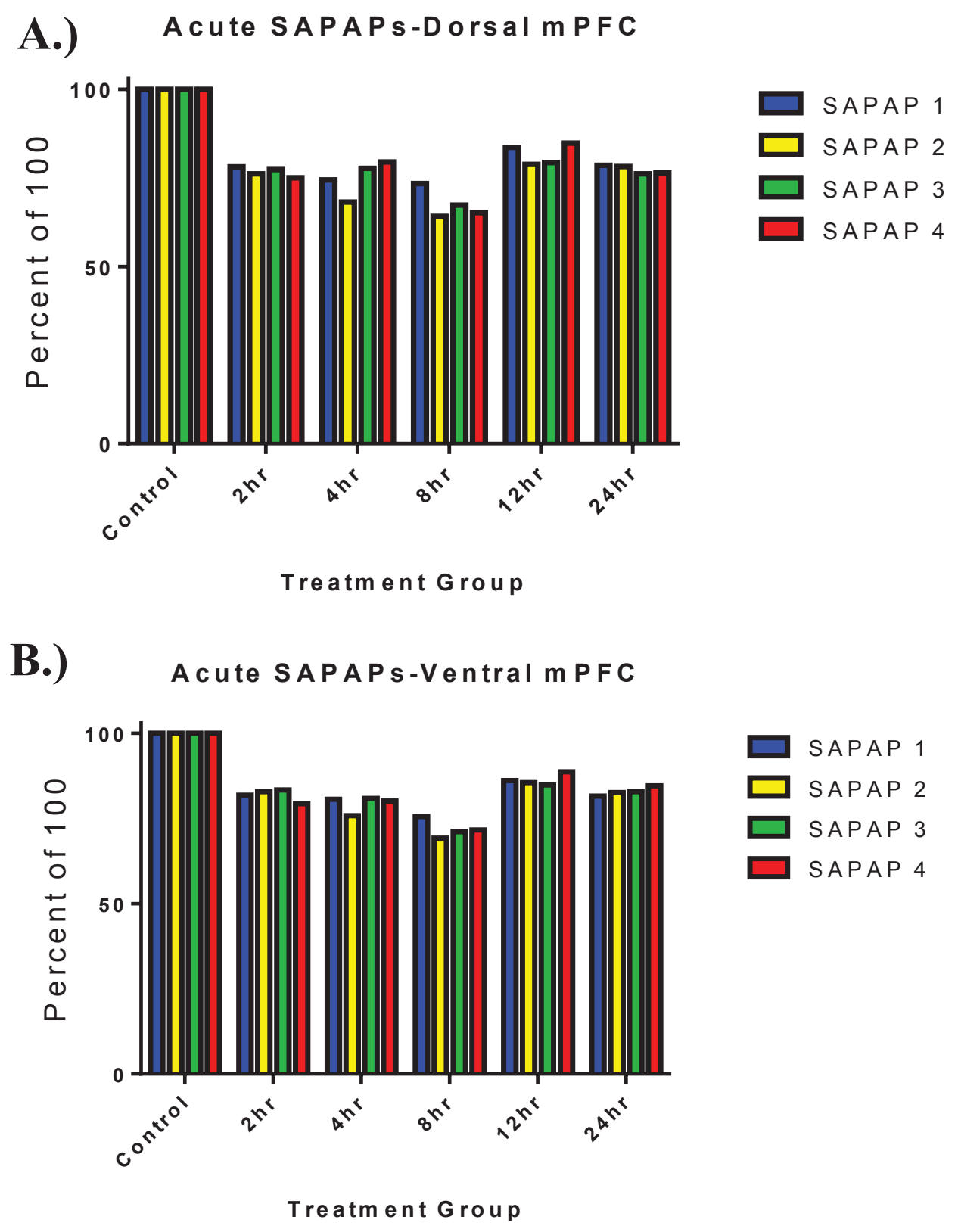

Figure 3-1. Acute Cocaine Exposure Induces a Transient Reduction in Prefrontal SAPAP Levels.

In the dorsal (top, A) and ventral (bottom, B) mPFC, protein levels for SAPAPs 1 through 4 , SAPAP levels trend towards a reduction at 2, 4, and 8 hours. This trend begins to return to baseline levels for cocaine naïve time-matched controls at 12/24 hours post cocaine exposure. 
Figure 3-2. Comparisons of Various Acute Time Points for SAPAPs 1 through 4 in the Dorsal and Ventral mPFC.

When all time points for a SAPAP/brain region are compiled and Compared to time matched cocaine naïve controls, SAPAPs display significant reductions at various time points after acute exposure (indicated by “*”). Significant reductions are observed only at $8 \mathrm{hr}$ post-exposure in the ventral $\mathrm{mPFC}$. In the dorsal $\mathrm{mPFC}$, this compilation displays significant reductions in SAPAP2 levels at the 2, 4, and 8 hours post exposure. For all experimental groups, $\mathrm{n}=4$. 


\section{Dorsal mPFC}

A.)

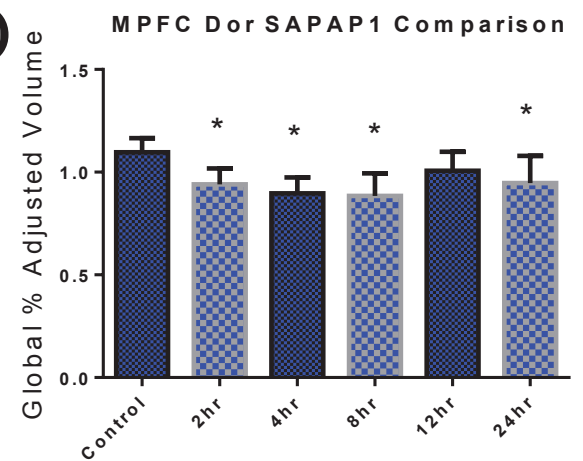

C.)

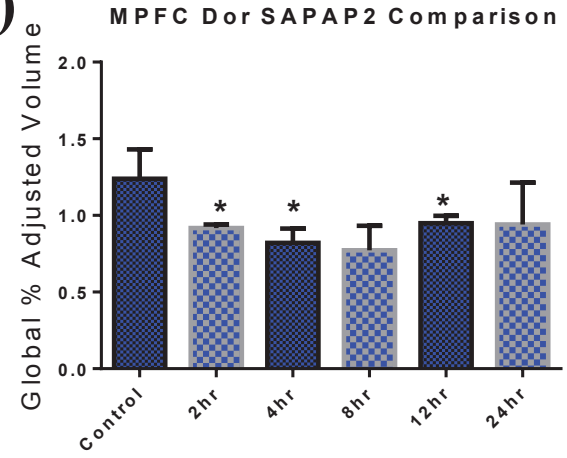

E.)

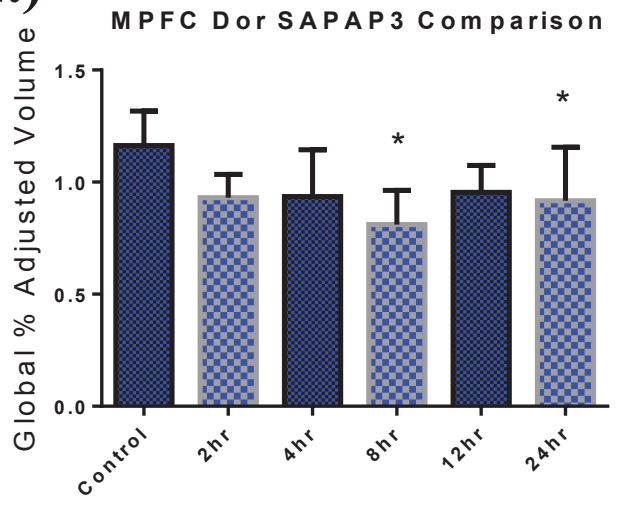

G.)

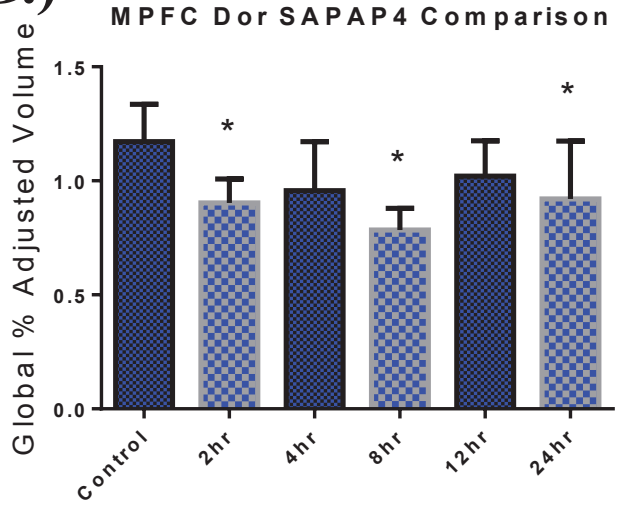

\section{Ventral mPFC}

B.)

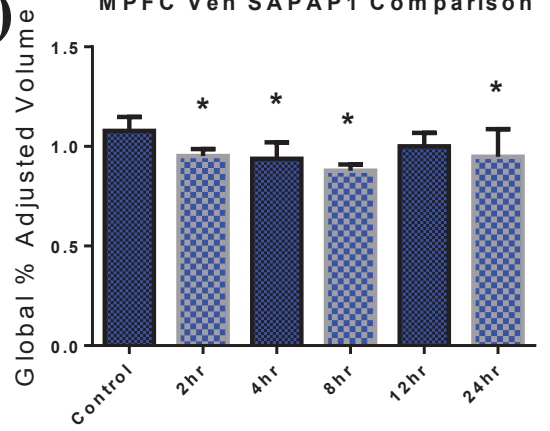

D.)

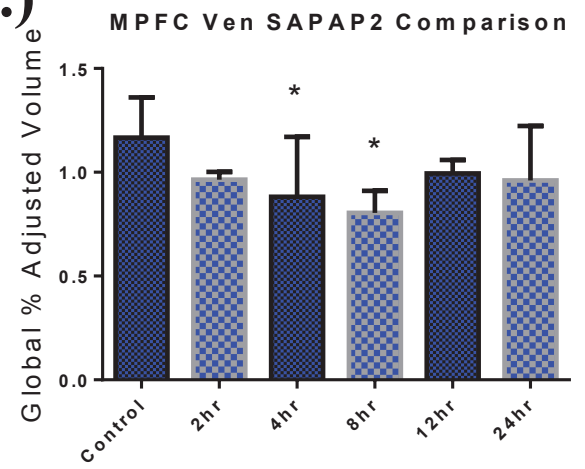

F.)

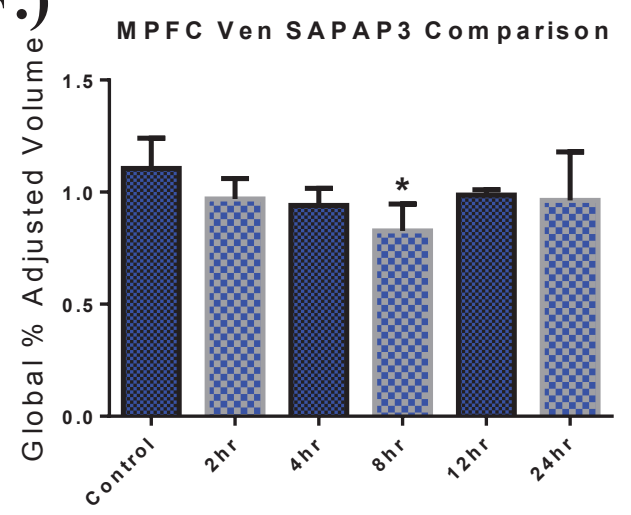

H.)

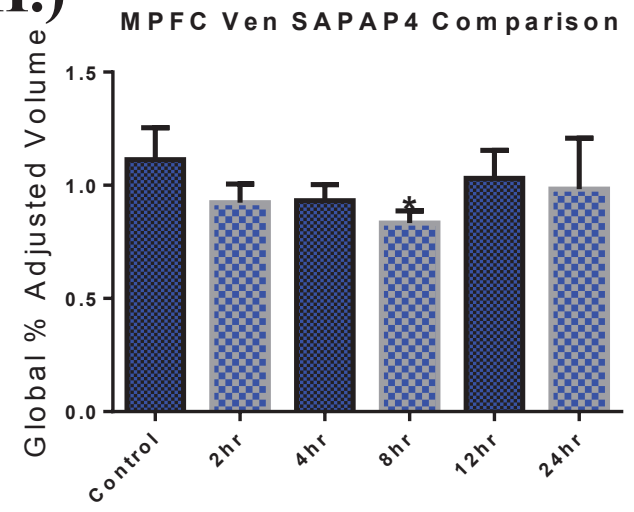


Table 3-1. Acute SAPAP Changes in Areas Other Than the mPFC.

\begin{tabular}{|c|c|c|c|}
\hline Area & SAPAP & Significant? & Time points affected- $(F, p)$ \\
\hline \multirow{4}{*}{ Nacc-CORE } & 1 & No & None \\
\hline & 2 & Yes & 2 Hours $(3.911,0.0079)$ \\
\hline & 3 & Yes & 2 Hours $(2.989,0.0244)$ \\
\hline & 4 & Yes & 2 Hours $(3.654,0.0079)$ \\
\hline \multirow[t]{4}{*}{ Nacc-SHELL } & 1 & No & None \\
\hline & 2 & Yes & 2 Hours $(3.195,0.0187)$ \\
\hline & 3 & No & None \\
\hline & 4 & Yes & 2 Hours $(6.211,0.0008)$ \\
\hline \multirow[t]{4}{*}{ STRIATUM } & 1 & No & None \\
\hline & 2 & No & None \\
\hline & 3 & Yes & $\begin{array}{l}2 \text { Hours }(5.261,0.0019) \\
12 \text { hours }(3.419,0.0142)\end{array}$ \\
\hline & 4 & No & None \\
\hline \multirow{4}{*}{$\begin{array}{l}\text { SUBSTANCIA } \\
\text { NIGRA }\end{array}$} & 1 & No & None \\
\hline & 2 & No & None \\
\hline & 3 & Yes & 2 Hours $(6.308,0.0003)$ \\
\hline & 4 & No & None \\
\hline \multirow{4}{*}{$\begin{array}{l}\text { VENTRAL } \\
\text { TEGMENTAL } \\
\text { AREA }\end{array}$} & 1 & No & None \\
\hline & 2 & No & None \\
\hline & 3 & No & None \\
\hline & 4 & No & None \\
\hline
\end{tabular}


The next set of experiments involved sensitized animals. After pretreatment, animals were verified to be sensitized using previously established protocols. Cocaine injected animals displaying sensitized locomotor responses over saline controls (Figure 3-3). For animals at 2-weeks and 1 day post-withdrawal, no significant differences were observed for any SAPAPs for any of the examined regions, or any differences between treatment groups

At the 3 week time point, a generalized statistically significant decrease was observed for SAPAPs 1-4 in the dorsal medial prefrontal cortex (Figure 3-4). In the dorsal mPFC, SAPAP1 was significantly decreased $(\mathrm{F}=6.793, \mathrm{df}=19, \mathrm{p}=0.0036)$ in all cocaine-exposed animals, compared to saline/saline animals (saline during daily treatments/saline on test day) that was even further reduced in cocaine sensitized animals receiving cocaine on test day (cocaine/cocaine). SAPAP1 was also decreased significantly $(\mathrm{F}=6.793, \mathrm{df}=19, \mathrm{p}=0.0036)$ for saline controls receiving cocaine on test day and sensitized animals receiving saline on test day, with even lower SAPAP levels observed in sensitized animals exposed to cocaine on test day. SAPAP2 was decreased significantly $(\mathrm{F}=3.800, \mathrm{df}=19, \mathrm{p}=.0312)$ in sensitized animals exposed to cocaine on test day when compared to cocaine naïve controls. SAPAP3 was decreased significantly $(\mathrm{F}=5.201, \mathrm{df}=19, \mathrm{p}=.0107)$ in sensitized animals receiving cocaine on test day when compared to cocaine-naïve animals. SAPAP4 was decreased significantly $(\mathrm{F}=7.421, \mathrm{df}=$ $19, \mathrm{p}=.0028)$ in all sensitized animals receiving cocaine on test day with even lower SAPAP levels observed in sensitized animals exposed to cocaine on test day. No significant differences were noted for SAPAPs in the ventral mPFC for this time point, all with ps $>.05$.

For our self-administration studies, all test and yoked animals maintained catheter patency throughout the entire experiment, and did not display injuries or infections before tissue harvesting. For test animals over the 10 day test period, significant or trending results were not observed for any of parameters of self-administration. SAPAP protein levels for test animals were observed to be significantly higher in both regions of the $\mathrm{mPFC}$ and Nacc core when compared to saline yoked control animals. In the dorsal mPFC (Figure 3-5A to $\mathbf{H}$ ) significant increases in SAPAP levels of test animals were observed for SAPAP1 $(\mathrm{t}=4.173, \mathrm{df}=6, \mathrm{p}=0.0059$, Figure 3-5A), SAPAP2 $(\mathrm{t}=3.909, \mathrm{df}=$ $6, p=0.0079$, Figure 3-5B), SAPAP3 $(\mathrm{t}=8.537, \mathrm{df}=6, \mathrm{p}=0.0001$, Figure 3-5C), and SAPAP4 $(\mathrm{t}=3.502, \mathrm{df}=6, \mathrm{p}=0.0128$, Figure 3-5D). In the ventral mPFC (Figure 3-5E) significant increases in SAPAP3 were observed $(t=2.756, d f=6, p=0.0330)$. In the Nacc core (Figure 3-5F and G) significant increases in SAPAP levels of test animals were observed for SAPAP2 $(\mathrm{t}=2.557, \mathrm{df}=6, \mathrm{p}=0.0431$, Figure 3-5F) and SAPAP4 $(\mathrm{t}=4.618$, $\mathrm{df}=6, \mathrm{p}=0.0036$, Figure 3-5G).

\section{Discussion}

When examining the time course of SAPAP changes in acutely exposed animals at many time points shorter than 24 hours, many significant SAPAP changes are found. 


\section{Cocaine Challenge}

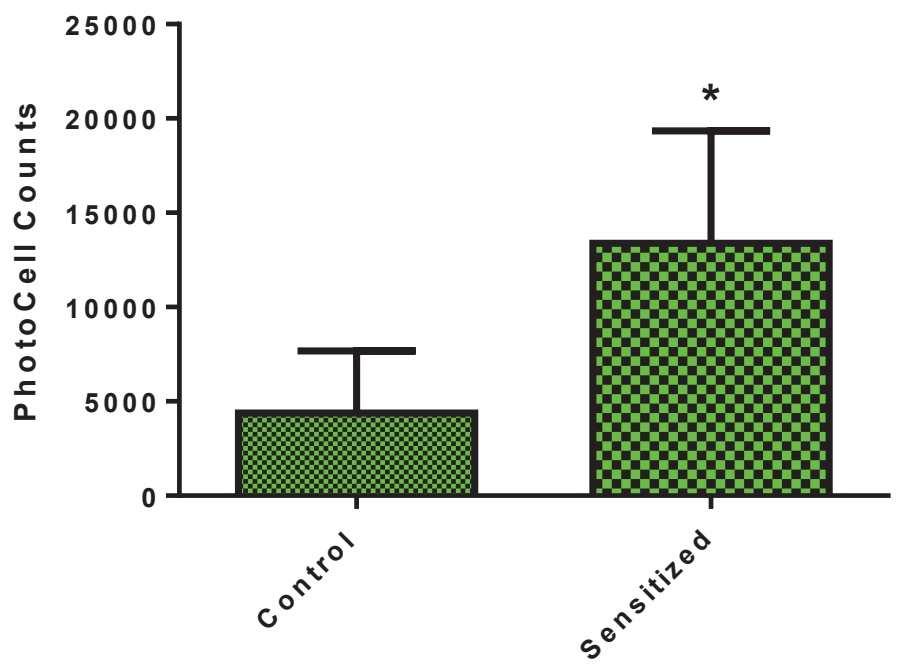

Figure 3-3. Sensitization Verification by Infrared Home Cage Monitors.

Testing included a $1 \mathrm{hr}$ activity baseline, followed by a $2 \mathrm{hr}$ activity recording that was immediately preceded by a challenge dose of $15 \mathrm{mg} / \mathrm{kg}$ cocaine I.P. injection. These results show a significant increase in photocell counts for sensitized animals receiving a challenge dose of cocaine over drug naïve control animals. For both experimental groups, $\mathrm{n}=16$. For sensitized animals, “*” indicates a significant change when compared to cocaine naïve animals. 

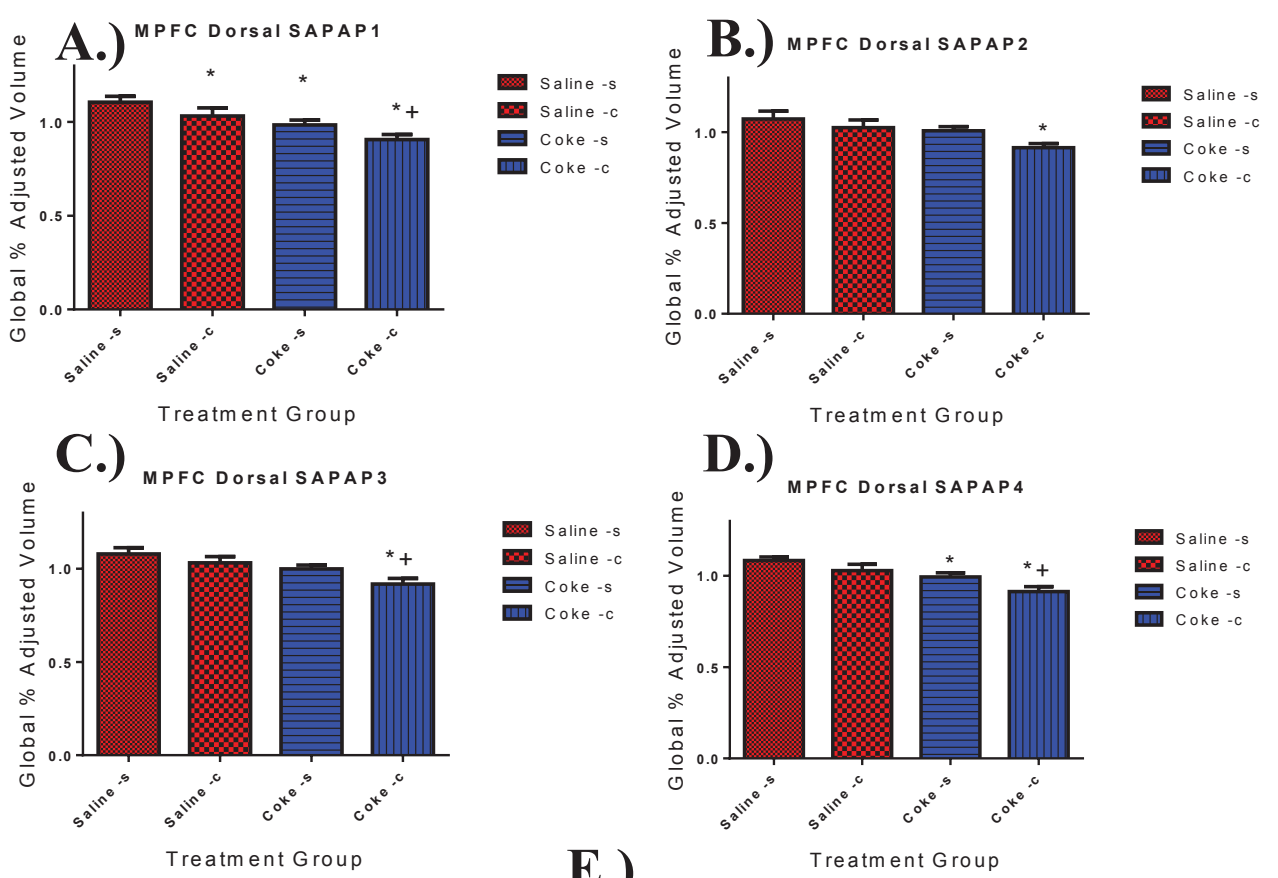

E.)

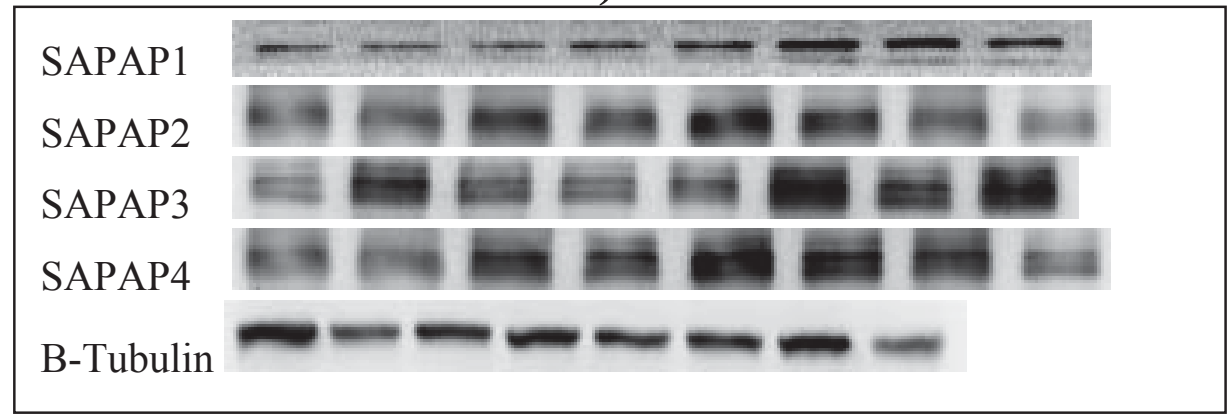

Figure 3-4. Varied SAPAP Reduction in Dorsal mPFC at 3 Weeks.

SAPAP levels in the dorsal mPFC are universally down regulated in response to cocaine exposure, although the statistical significance and pattern of this down regulation varies. SAPAP1 (upper left, A), is decreased in similar fashion in cocaine naïve animals acutely exposed to drugs on test day and animals completely abstinent for 3 weeks, although an acute dose drive SAPAP1 levels even lower. SAPAP2 (upper right, B) are only significantly decrease in sensitized animals exposed to drug after 3-weeks on test day, which is similar in fashion to SAPAP3 levels (lower left, C). SAPAP4 levels in the mPFC (lower right, D) are statistically lower only in sensitized animals; levels are lower in animals receive cocaine on test day than animals experiencing a 3-week withdrawal, compared to cocaine naïve controls. Unaltered representative blot images are shown in panel E. For all experimental groups, $n=4$. For all panels, “*” indicates a significant change from saline naïve animals, and "+" indicates a significant change from acutely exposed saline animals. 
Figure 3-5. SAPAP Levels of the Dorsal mPFC Are Elevated for All SAPAPs in Test Self-Administration Rats.

Protein levels for SAPAP1 (upper left, A), SAPAP2 (upper right, B) SAPAP3 (lower left, C), and SAPAP4 (lower right, D) in the dorsal mPFC are significantly increased for test animals self-administering cocaine $(.3 \mathrm{mg} / \mathrm{kg} /$ infusion $)$ when compared to yoked cocaine naïve controls receiving saline. SAPAP3 levels (middle, E) are increased in the ventral $\mathrm{mPFC}$ of test animals self-administering cocaine $(.3 \mathrm{mg} / \mathrm{kg} /$ infusion $)$ when compared to yoked cocaine naïve controls receiving saline. In the Nacc core of these same animals, a similar significant increase in SAPAP2 (bottom left, F) and SAPAP4 (bottom right, G). For all experimental groups, $\mathrm{n}=4$. For all panels, “*” indicates a significant change from yoked animals. 


\section{3 week abstinence period following cocaine self}

administration
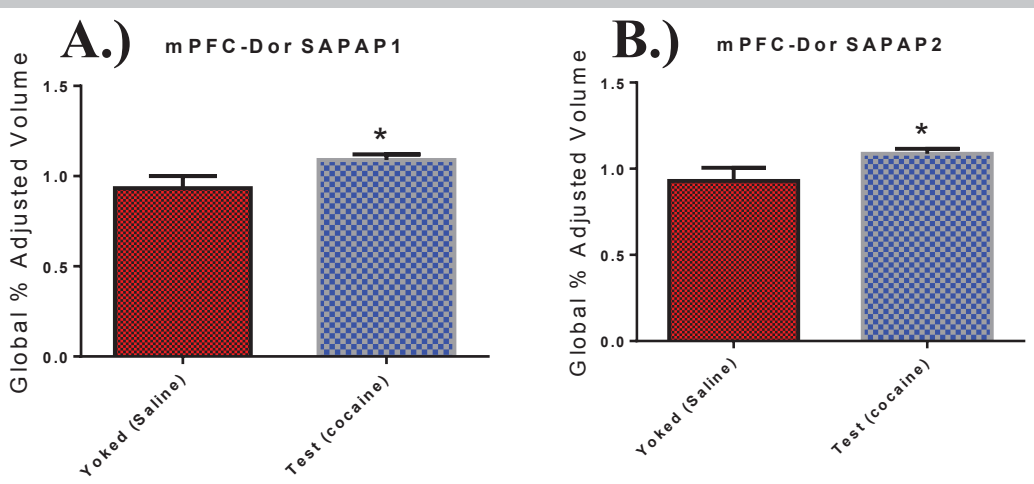

C.) mpfc-dorsapap3
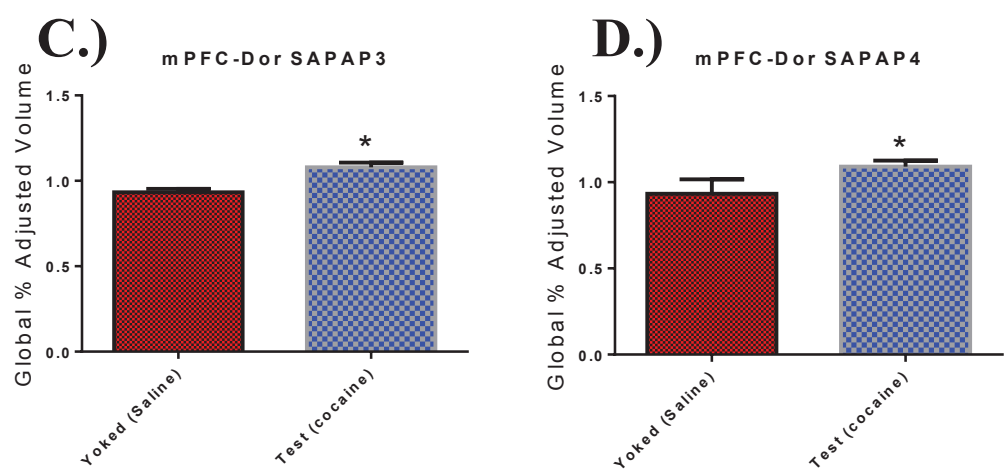

E.) mPFC-Ven SAPAP3

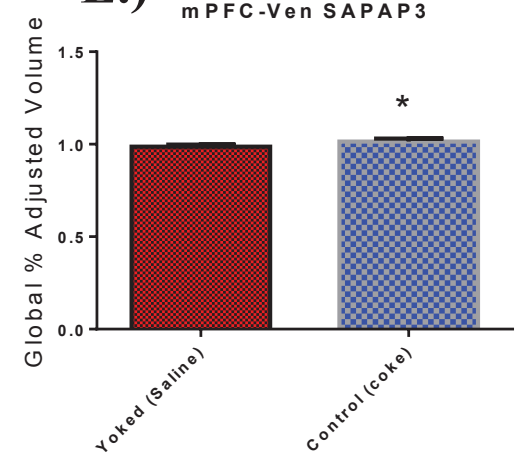

F.)

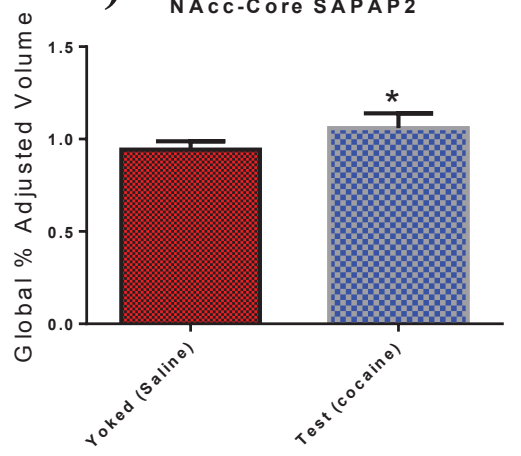

G.) nacc-core sapap4

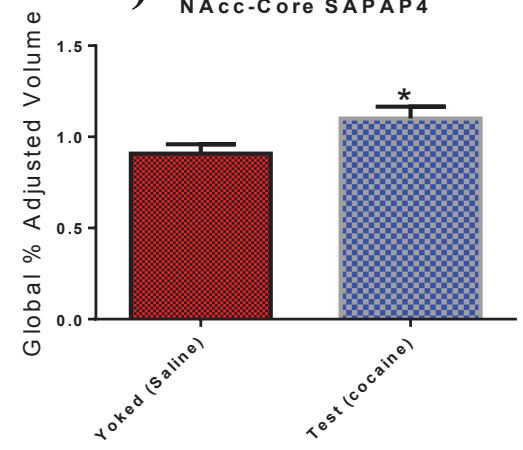


These changes occur in most regions examined, and in most cases are not significant at 24 hours after an acute dose of cocaine. This indicates that while far reaching, these changes along with their effects may be transient. Yet, in sensitized animals a particular subset of alterations becomes permanent. Interestingly, the striatum, substantia nigra, and VTA are not altered in the sensitization process, and in acute animals these regions display a significant elevation in certain SAPAPs. In contrast to the general SAPAP levels in these acutely and non-contingently exposed animals, cocaine-naïve animals that are acutely exposed to cocaine after 10 days of self-administration display universally elevated SAPAP levels. Although these changes may also be transient, significant SAPAP changes involve regions that are critical to maintenance of cocaine sensitization. In the dorsal prefrontal cortex, all 4 SAPAPs are significantly increased, while in the ventral $\mathrm{mPFC}$ only SAPAP3 is increased. For the self-administration experiments, the Nacc core of test animals also displays a significant increase in SAPAP 2 and SAPAP 4.

For all other brain regions examined for all SAPAPs at the 1 day/2-weeks post abstinence time point, no statistically significant changes were observed. Differing from this pattern, results at 3-weeks post sensitization time point were predicted to yield the most changes in SAPAPs, as sensitization related molecular changes tend to be more exaggerated for the 3 week time period (expression phase) when compared to early time periods (initiation phase) [154]. Significant changes for the 1 day and 2 week time point yield no significant results (data not shown). However, at the 3 week time point, SAPAP reduction occurred in a variety of patterns for SAPAPs examined in the dorsal prefrontal cortex, along with a more consistent pattern of SAPAP protein deficiency observed in the Nacc core. This may be explained by the finding that at 3 week of cocaine abstinence after the last daily injection to induce sensitization, the sensitized locomotion displayed by sensitized animals in response to cocaine can be mimicked by AMPA injections into the Nacc core [155]. This effect is not due to AMPA-induced dopamine release [155]. As AMPA is an artificial agonist that stimulates a specific class of ionotropic glutamate receptor, endogenous glutamate released in this region would also stimulate NMDA-type receptors, which removes SAPAPs from the synapse [138]. In the dorsal mPFC, all SAPAPs were significantly reduced in animals sensitized to cocaine and acutely exposed on test day. Moreover, for all time points, while the ventral prefrontal cortex, nucleus accumbens shell, dorsal striatum, substantia nigra, and ventral tegmental area were examined for all 4 SAPAPs, no other areas displayed statistically significant differences.

The decrease in SAPAP levels for the majority of brain regions is a promising step in determining that extent of the molecular changes that occur in long-term cocaine abuse, and a possible mechanism to explain the compulsivity associated with cocaine addiction. Considering that SAPAPs are scaffolding proteins that help traffic NMDA receptors and PSD-95 to the synapse, these results may help explain why either NMDA receptor number or functionality are affected, as the absence of these proteins (especially acutely) could thwart efficient intracellular signaling. Indeed, the same publication that found SAPAP3 deletion is sufficient to cause compulsive grooming also reported that the same mice showed defects in neurotransmission, specifically reduced post synaptic field potentials of NMDA receptors [89]. Although our results reported here do not attempt to test physiology in any way, the loss of SAPAPs, with their role in facilitating clustering 
of NMDA receptors, may help to further explain the glutamatergic disturbances in the sensitized brain [84]. Additionally, in the 3-week abstinence cohort, the results of the significant decrease of SAPAP protein in both the dorsal mPFC as well as the Nacc core fit comfortably in a larger context of addiction circuitry, as the dorsal $\mathrm{mPFC}$ specifically innervates the Nacc core, while enhanced behavioral sensitization is associated with enhanced glutamate transmission to this region [156]. Considering that cocaine sensitization results in increased glutamate transmission, the finding that SAPAP levels tend to be decreased also with cocaine exposure is not surprising. This is because excitation is known to remove SAPAPs from the synaptic density [138]. Moreover, the lack of significant SAPAP alterations at the 2-week and 1-day time points was partially expected, as certain facets of behavioral sensitization caused by altered glutamate transmission may simply require a longer incubation period to occur than examined here. This process continues to develop for up to 2 months in rats [157], and perhaps up to a full year in humans exposed to psychostimulants [158].

Indeed, this extended time course of glutamatergic changes may partially account for the SAPAP changes we found in rats responding for cocaine. In these animals, for all significantly altered regions SAPAPs are universally elevated over cocaine naïve yoked controls. Although cocaine sensitized animals acutely exposed on test day display slightly lower protein levels, SAPAP levels in acutely exposed controls or sensitized animals unexposed on test day are slightly higher. While not definitive, these results may indicate that short incubation time following acute exposure to cocaine may result in increased SAPAP levels. Moreover, the finding that all SAPAPs in the dorsal $\mathrm{mPFC}$ and dendritic SAPAP3 in the ventral $\mathrm{mPFC}$ are altered may reflect the unequal roles of these regions in the sensitization process, as changes occur early in the dorsal mPFC and later in the ventral $\mathrm{mPFC}$ as cocaine exposure progresses from acute to chronic.

For future experiments addressing our results, we will extend these studies to a later time point, and examining if protein localization is affected in cocaine sensitized/exposed rats. This may yield additional data into how sensitization affects SAPAP levels. We also hope to use viral constructs to knockdown SAPAP mRNA expression in the key regions studies, to determine if this alone would have any effect on the behavioral aspects of sensitization. However, if not by conducted by our group, molecular studies into the exact roles of SAPAPs would greatly enhanced the impact of this and any future experiments as the intimate details of the role of this protein family are largely a mystery. Part of this mystery includes the lack of a mechanism to explain why this occurs. One possible theory is that mechanism controlling SAPAP levels may also include proteins that regulate gene expression. Cocaine can selectively activate class II activity-dependent histone deacetylases (HDACs), especially HDAC5. When activated exposing normally inactivated regions of chromatin to the possibility of transcription [159]. This is not a very abstract line of thought. HDAC5-deficient mice show a strong preference for the rewarding effects of cocaine in a conditioned place preference paradigm (which is rescued by viral transfection of full-length native HDAC5), and that HDAC5 is associated with enhanced cocaine responses [159]. To confirm this theory, brain tissue of animals confirmed to be sensitized could also be probed for H3K9/14Ac, and H4K5/8/12/16Ac, known markers of transcription activation [160]. 


\section{CHAPTER 4. SENSITIZATION-INDUCED CHANGES OF AMPA/NMDA RECEPTOR RATIO IN THE MPFC}

\section{Rationale}

Population changes of ionotropic AMPA/NMDA glutamate receptors are critical to the initiation and maintenance of cocaine sensitization. In response to cocaine sensitization, these changes have been reported to be enduring in the nucleus accumbens (Nacc), transient in the ventral tegmental area (VTA), and currently unreported in a region known to be dysfunctional in cocaine addicts, the medial prefrontal cortex (mPFC). Here, we examined the effects of cocaine sensitization and acute cocaine exposure on the AMPA/NMDA receptor pool in the dorsal and ventral mPFC. Rats were sensitized to cocaine over 4 days $(15 \mathrm{mg} / \mathrm{kg} /$ day $)$ and along with time-matched shaminjected controls, exposed to an acute dose or saline injection after a 1 day, 2 weeks, or 3 weeks cocaine abstinence period. Using this paradigm, several acute and sensitizationrelated changes were observed. After 2-weeks of abstinence, changes uncovered included a decrease in dorsal GluR1 subunits, an increase in ventral intracellular GluR2 subunit, and a decrease in intracellular NR1 subunits. At 3 weeks of abstinence, significant receptor changes were restricted to the ventral $\mathrm{mPFC}$, and consisted of a significant increase in GluR2 and NR2B surface subunits in sensitized animals. These results will broaden our understanding of how glutamate transmission is affected by acute cocaine exposure and cocaine sensitization.

\section{Background}

In comparison to dopamine, glutamate has proven to be a vital, yet often upstaged actor in the interplay of the neurotransmitters that contribute to cocaine sensitization. In cocaine users, many symptoms associated with long-term cocaine use can be directly traced to altered glutamate transmission. Evidence implicating glutamate has been found in live addicts [161] and cadaver tissue [162], with much of the data collected in clinical settings being confirmed in animal models [163]. Live brain imaging indicates decreased prefrontal activity as well as decreased prefrontal glutamate levels in cocaine addicts even after periods of abstinence [71]. Furthermore, severing the glutamatergic projections from the medial prefrontal cortex (mPFC) in rats [164] or selective blockade of excitatory glutamate receptors [165] will halt the expression of any previously established behavioral sensitization. In humans, changes in glutamate receptors are thought to be responsible for the escalation of drug use that demarcates recreational and habitual use [166]. Moreover, long-term chronic cocaine use is thought to result in behavioral sensitization, a disorder marked by cocaine induced psychosis [167], paranoid behavior[168], seemingly useless repetitive movements [169], intrusive/unwanted thoughts about drugs/drug cravings [170], and relapse even after a long period of healthy sobriety [170]. Although these effects are thought to stem from changes in prefrontal glutamate, the mechanisms of how the populations of AMPA/NMDA ionotropic glutamate receptors (iGluRs) are affected in this region is currently a mystery. Despite 
this, it is known that the end result of cocaine-induced changes in the mPFC creates a region hyper-excitable to glutamate, indicating that there may be an increase in the contribution of excitatory AMPA receptors [171]. This enhanced response to glutamate in not solely restricted to the $\mathrm{mPFC}$, and thus the study of other regions of this mesocorticolimbic system may reveal how glutamate receptors are altered in this region.

Considering that individual brain regions work as a circuit to control behavior, it is reasonable that the process of altered glutamate transmission in the $\mathrm{mPFC}$ is initiated elsewhere. In response to cocaine sensitization, both the "core" and more lateral "shell" of the Nacc display an abnormally high synapse-to-neuron ratio [172]. These extra synapses are the result of structurally abnormalities that include increased dendritic spines, dendrites with multiple spines, longer than average dendrites, and dendrites that inappropriately "boundary cross" into adjacent nuclei [76]. This underlying structural remodeling in the Nacc core ultimately influences the physiology of the Nacc, and is initiated by NMDA receptors [77]. The Nacc Core occupies a critical junction in the mesocorticolimbic system as there is a direct connection between it and the dorsal portion of the $\mathrm{mPFC}$ where critical exchanges of neurotransmitter innervations occur.

In response to cocaine at this critical junction, a series of events follows that is critical to the development of behavioral sensitization. In response to repeated cocaine exposure, dopamine release in the $\mathrm{mPFC}$ is reduced [173] and results in behaviors reminiscent of those observed in cocaine sensitized animals [174]. Glutamate levels in the $\mathrm{mPFC}$ are concurrently elevated, and glutamate antagonist in the $\mathrm{mPFC}$ will prevent sensitization [175]. Moreover, although cocaine sensitization affects the $\mathrm{mPFC}$ as a whole, the dorsal and ventral regions are affected differently by cocaine sensitization. The dorsal $\mathrm{mPFC}$ is preferentially affected in response to acute cocaine, and lesioning this area will prevent the initiation and expression of sensitization from occurring [147]. Conversely, administering the GABA agonist baclofen in the ventral $\mathrm{mPFC}$ will prevent acute cocaine-induced motor activity and initiation of sensitization, but not its expression [148].

Considering that both the Nacc and mPFC show enhanced excitability to glutamate, underlying mechanisms within these two regions may be similar. Although unknown for the $\mathrm{mPFC}$, the manner in which glutamate receptors are altered in the Nacc is well documented for fast-acting ionotropic glutamate receptors (iGluRs). These receptors directly affect current influx, while slower-acting metabotropic glutamate receptors (mGluRs) moderate neuron-wide responses and iGluRs activity [176]. Sensitization-induced glutamate sensitivity in the Nacc occurs due to an increased contribution of $\mathrm{Ca} 2+$ permeable AMPA receptors (CP-AMPARs) [177]. This effect is also concurrently associated with a decreased contribution of LTP-inhibiting mGlur1 and NMDA receptors $[178,179]$. NMDA receptors themselves are reduced in response to psychostimulant withdrawal in the Nacc [180], with the resulting AMPA-dominant current contributing to the incubation of craving and vulnerability to cue-induced relapse. This AMPA current is also associated with increased expression, total input strength, and accumulation of CP-AMPARs [181]. In addition to these effects on iGluRs, the ability of mGluR1 to mediate LTD is decreased in the Nacc. Considering this, not only does 
receptor content influence output from nuclei/subnuclei, but output may also be influenced by subunit composition of these receptors. This is because subunit composition determines whole-receptor function. For example, CI-AMPARs lose their permeability to calcium through inclusion of GluR2 [182]. NMDA receptors require the NR1 subunit as a necessary component to generate typical NMDA-specific currents [183], and have NR2 subunits with cell-type specific intracellular C-terminal domains that alter channel kinetics [184]. The activity-dependent ratio of NR2A/NR2B is vital to cortical functioning [185], and in response to increased activation of NMDA receptors, NR2A subunits are decreased concurrently with receptor activity [186] (an effect that is $\mathrm{Ca} 2+$ dependent [187]). These subunits can also be increased by blockade of iGluRs and/or cell activity [188], indicating that down-regulation of these subunits may be protective against the harmful effects of increased glutamatergic transmission. NR2B subunits do not appear to be dynamically regulated, and although NR2A/B ratio is critical, this ratio appears to be solely regulated through NR2A-subunit alterations [188]. Considering that NR2A/B are expressed at their highest levels in the cerebral cortex [70], it stands to reason that sensitization-induced iGlurR population changes may have a significant role in the $\mathrm{mPFC}$.

Taken these studies of the mPFC and Nacc together, a model emerges that adds a functional context to the consequences of modifications to the mesocorticolimbic circuit in response to cocaine sensitization. In this model, dramatic yet transient changes occur in the VTA that increase the dopaminergic tone onto the Nacc. Glutamate receptor changes result in a regional hyper excitability due to an increased contribution of $\mathrm{Ca} 2+-$ permeable AMPA receptors, but also a lack of glutamatergic braking ability due a decreased contribution of mGlur1 and NMDA receptors. Taking these facts into account along with studies that show decreased activity [71] as well as decreased prefrontal glutamate levels in the prefrontal cortex of cocaine addicts even after abstinence indicate that glutamate receptors could play a vital role in the long term expression. We believe this is region ripe for investigation, and here we propose to investigate if behavioral sensitization to cocaine results in changes of whole surface AMPA/NMDA receptor and receptor subunit populations in the $\mathrm{mPFC}$, and the time course of any changes.

\section{Methods}

\section{Animal Subjects}

48 male albino Sprague Dawley rats weighing approximately $250 \mathrm{~g}$ on arrival were acclimated to reversed light cycle housing (LIGHTS OFF - 05:00-18:00 hrs, LIGHTS ON- 18:00-05:00 hrs) for 3 days, and were housed individually for the duration of their lives. Rats were sensitized by receiving an injection of cocaine $(15 \mathrm{mg} / \mathrm{kg})$ once a day at 12:00-13:00 for 4 days. A separate group control animals received sham (saline) injections on the same schedule as sensitized test animals. Next, both groups of animals were divided into 3 cohorts that underwent a period of abstinence for 1 day, 14 days, or 21 days (food/water free-feeding). On the day following the abstinence period, sensitized 
animals were tested alongside time-matched and sham-injected control rats for any enhanced drug induced locomotion in infrared home cage monitors (Accuscan Instruments Inc.). A baseline measure of motor activity was obtained for $1 \mathrm{hr}$, and then sensitized animals either were challenged with a dose of $15 \mathrm{mg} / \mathrm{kg}$ I.P. cocaine (cocaine/cocaine group) or received a sham injection of saline (cocaine/saline group). Cocaine naïve controls were examined alongside sensitized animals, and either received a single injection of cocaine on test day (saline/ cocaine group) or another injection of saline (saline/saline). Regardless of the injection administered, the challenge session was concluded after $2 \mathrm{hr}$. Immediately following the end of the challenge session, rats were anaesthetized in a sealed gas chamber by introduction of pure $\mathrm{CO} 2$ for $10 \mathrm{sec}$ and immediately sacrificed by guillotine.

\section{Tissue Harvesting and Crosslinking}

After removal, the brain was placed on ice then dissected for the medial prefrontal cortex (mPFC, dorsal and ventral regions separated), using established protocols [151]. Immediately after the removal of each brain region using ice-cold instruments without freezing the brain tissue before dissection, specimens were manually "diced" into pieces. All samples cut consistently in approximately the same manner and size for each animal. Sample pieces were placed in freshly prepared, ice-cold artificial cerebrospinal fluid (1.2 mM CaCl2, 20 mM HEPES-pH 7.4, 0.147 M NaCl, 2.7 mM KCl, 1 mM MgCl2, 100 $\mathrm{mM}$ Dextrose) spiked with $52 \mathrm{mM}$ bis-sulfosuccinimidyl suberate (BS3) immediately before the addition of tissue to cross-link surface AMPA/NMDA receptors. Next, samples were left to agitate at $4^{\circ} \mathrm{C}$ for 30 minutes, and after this period sample tubes were next spiked with 100 ul of $1 \mathrm{M}$ glycine solution to quench BS3, and incubated for an additional 10 minutes at $4^{\circ} \mathrm{C}$. After incubation, all samples were centrifuged for 2 min at 20,000 X g. The supernatant was then discarded. Less than 3 min elapsed from

guillotining to sample incubation in artificial cerebrospinal fluid. For longer term storage, samples were stored at $-80^{\circ} \mathrm{C}$.

\section{Protein Sample Preparation and Immunoblotting}

Whole tissue samples were sonicated in 2\% SDS and centrifuged at 13,000 X g for $30 \mathrm{sec}$. To obtain the protein concentration of the sample, $200 \mu 1$ of this whole tissue sample preparation was analyzed in triplicate using a Bio-Rad ${ }^{\circledR}$ DC ${ }^{\text {TM }}$ Protein Assay Kit. The triplicate readings were average together, and this average was used as the protein concentration. An aliquot of tissue sample was diluted to $2.0 \mu \mathrm{g}$ protein/ $\mu 1$ with $2 \%$ SDS. An aliquot of this diluent was diluted to $1 \mu \mathrm{g}$ protein/ $\mu \mathrm{l}$ with an equal volume of $2 \mathrm{x}$ electrophoresis sample buffer [final concentration after dilution $=50 \mathrm{mM}$ Tris $\mathrm{HCl}(\mathrm{pH}$ 6.7), $4 \%$ glycerol (wt/vol), 2\% SDS, $5 \mathrm{mM}$ dithiothreitol and bromophenol blue (0.01 $\mathrm{mg} / \mathrm{ml})]$. Proteins $(20 \mu \mathrm{g} / \mathrm{lane})$ were separated by size on a $10 \%$ SDS-polyacrylamide gel transferred to Immobilon-P PVDF transfer membranes (Millipore) in Towbin's-SDS transfer buffer (25 mM Tris, $192 \mathrm{mM}$ glycine, 20\% methanol, and $0.01 \%$ SDS) at $120 \mathrm{~V}$, $0.35 \mathrm{~A}$ for $2 \mathrm{hrs}$ at room temperature with the transfer apparatus packed in ice. After 
transfer, the membrane was removed from the transfer apparatus, was left to dry overnight at room temperature.

Membranes were reactivated after $12 \mathrm{hrs}$ using 1x $20 \mathrm{sec}$ methanol wash, followed by 2x $30 \mathrm{sec}$ wash in ddH20, 1x 10 min wash in ddH20, and 1x 15 min wash in TBST. Membranes were blocked in TBST containing 5\% nonfat dried milk (NFDM Blotting Grade Blocker, Bio-Rad) for $1 \mathrm{~h}$ at room temperature with agitation. The membrane was washed once with TBST for 5 min and incubated with the primary antibody (subunits GluR1, GluR2, NR1, NR2A, and NR2B obtained from Sigma-Aldrich Company, St. Louis MO) diluted to 1:20,000 in TBST containing 0.5\% NFDM for $1 \mathrm{~h}$. As an experimental control, all throughout the labeling process, another portion of each corresponding membrane was concurrently probed with an anti-beta tubulin antibody (Sigma Aldrich). The membranes were then washed once for $15 \mathrm{~min}$ and twice more for $5 \mathrm{~min}$ in TBST followed by a $1 \mathrm{hr}$ incubation with agitation at room temperature with horseradish peroxidase-conjugated goat anti-rabbit immunoglobulin G (Pro-Sci) diluted 1:5000 in TBST containing 0.5\% NFDM. After this incubation, the membranes were washed as above and the antigen-antibody-peroxidase complex was detected by enhanced chemiluminescence (West Femto Substrate, Thermo Scientific) according to the manufacturer's instructions and visualized, analyzed and quantified by computer-assisted densitometry using a Bio-Rad Molecular Imaging System using Quantity One Imaging Software. After visualizing, the membranes were then stripped using $0.1 \mathrm{M}$ glycine stripping buffer $(\mathrm{pH}=2.2)$ containing $1 \%(\mathrm{v} / \mathrm{v})$ Tween-20.

\section{Analysis}

For each subunit or beta tubulin band, the band area was measured and using the imaging software, its adjusted percent volume was calculated, globally subtracting any background present. For each subunit band, adjusted percent volume value was then divided by its corresponding beta tubulin value using Microsoft Excel for each sample lane. External whole receptors were measured as the top band observed to not run into the lower separating gel, and internal subunits as bands small enough to run into the stacking gel, ranging in size from approximately $80-130 \mathrm{kDa}$. All statistical calculations were performed using Prism (Version 5, Graphpad Software Inc.) one-way ANOVA analysis (Newman-Keuls multiple comparison post-test)

\section{Results}

After pretreatment, animals were verified to be sensitized using previously established protocols $(t=5.492, d f=30, p=<0.0001$, Figure 4-1). Subunits examined for NMDA receptors consisted of NR2A, NR2B, and NR1. The NR1 subunit is a necessary subunit for NMDA receptors, and any changes associated with this subunit were considered to represent a change in PSD receptor numbers. Subunits examined for AMPA receptors consisted of GluR1 and GluR2. The GluR1 subunit is a necessary subunit for AMPA receptors, and any changes associated with this subunit were 


\section{Cocaine Challenge}

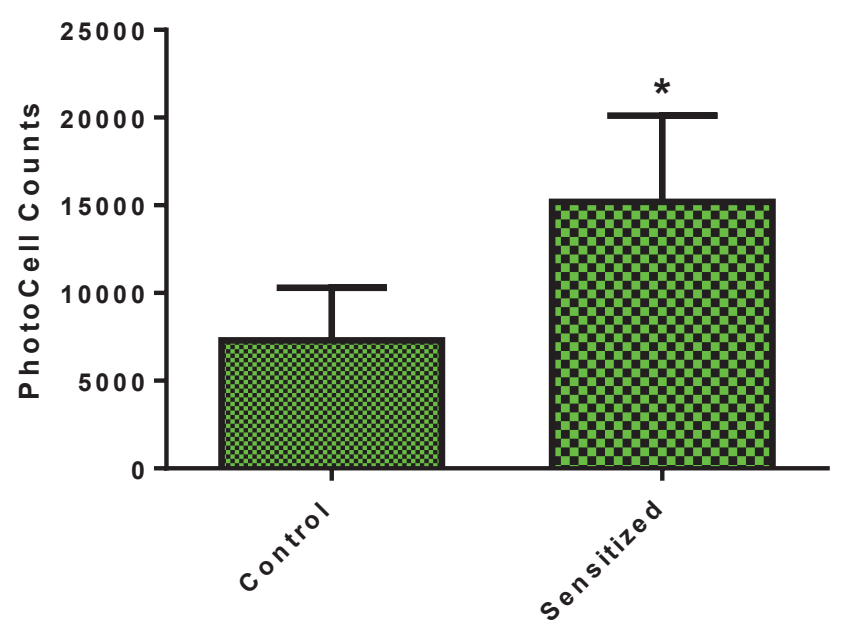

Figure 4-1. Sensitization Verification by Infrared Home Cage Monitors.

Testing included a $1 \mathrm{hr}$ activity baseline, followed by a $2 \mathrm{hr}$ activity recording that was immediately preceded by a challenge dose of $15 \mathrm{mg} / \mathrm{kg}$ cocaine I.P. injection. These results show a significant increase in photocell counts for sensitized animals receiving a challenge dose of cocaine over drug naïve control animals. For both experimental groups, $\mathrm{n}=16$. For sensitized animals, “*” indicates a significant change when compared to cocaine naïve animals. 
considered to represent a change in PSD receptor numbers. Significant receptor changes for either AMPA or NMDA receptors, as well as subunits, are not present for any treatment group at 1 day of withdrawal after sensitization injections and subsequent verification, with zero trending results, and wide inter/intra treatment group variances noted (data not shown: $\mathrm{p}>0.05$ ).

AMPA receptor subunits were also examined at 2 and 3 weeks of abstinence. For AMPA receptor subunits at 2 weeks post withdrawal compared to cocaine-naïve controls, a significant reduction for GluR1 subunits was observed in the dorsal mPFC for the intracellular and extracellular fractions. In the intracellular fraction, significant differences were observed for saline/saline vs. saline/cocaine, saline/saline vs. cocaine/saline, and saline/saline vs. cocaine/cocaine $(\mathrm{F}=10.88, \mathrm{df}=15, \mathrm{p}=0.0010$, Figure 4-2A). Sensitized animals acutely exposed on test day displayed further reduction in GluR 1 when compared to the saline/cocaine group and the cocaine/saline group. In the extracellular fraction $(\mathrm{F}=20.65, \mathrm{df}=15, \mathrm{p}=<0.0001$, Figure 4-2B $)$, significant differences were observed for saline/saline vs. saline/cocaine, saline/saline vs. cocaine/saline, and saline/saline vs. cocaine/cocaine. Sensitized animals acutely exposed on test day displayed further reduction in GluR1 when compared to the saline/cocaine group and the cocaine/saline group. The ventral $\mathrm{mPFC}$ displayed no significant changes in GluR1 for this time period in expressed receptors or internal subunits (data not shown, $\mathrm{p}>.05$ ). For both fractions of the dorsal mPFC, GluR2 subunits were insignificantly altered for this time point (data not shown, $\mathrm{p}>.05$ ), and for the external fraction of for the ventral mPFC (Figure 4-2D). For the internal subunits of GluR2 in the ventral mPFC, GluR2 subunits are significantly elevated with significant differences observed for saline/saline vs. saline/cocaine, saline/saline vs. cocaine/saline, and saline/saline vs. cocaine/cocaine $(\mathrm{F}=29.02, \mathrm{df}=15, \mathrm{p}=>.0001$, Figure 4-2C). Sensitized animals regardless of exposure on test day also displayed an increase in GluR2 when compared to the saline/cocaine group, for the cocaine/saline group and the cocaine/cocaine group. At the 3-week withdrawal time point, a significant elevation surface GluR2 subunits was observed for the cocaine/saline and cocaine/cocaine groups when compared the drug naïve controls $(\mathrm{F}=3.521, \mathrm{df}=23, \mathrm{p}=0.0339$, Figure 4-3).

Concerning NMDA receptors at 2 weeks withdrawal, while no significant changes were recorded for the dorsal mPFC in NR1 levels (data not shown), we observed a significant reduction of NR1 subunit in the internal fraction of the ventral $\mathrm{mPFC}$ $(F=14.62, d f=15, p=0.0003$, Figure 4-4A). When compared to naïve controls, significant differences are observed for the saline/cocaine group and cocaine/saline group, but not for the cocaine/cocaine group. No significant differences were observed for the surface fraction (Figure 4-4B). For the NR2A subunit at 2 weeks of abstinence, results followed similar patterns in both the dorsal and ventral $\mathrm{mPFC}$, although statistically significant changes were only found in the ventral mPFC. For the internal fraction, a significant elevation in NR2A levels was observed with comparable differences for the saline/cocaine group and cocaine/saline group when compared to naïve controls $(\mathrm{F}=11.21, \mathrm{df}=15, \mathrm{p}=0.0009$, Figure 4-4C). Conversely, there was a significant reduction in NR2A in sensitized animals acutely exposed on test day when compared to the saline/cocaine group and the cocaine/saline group. Results were similar 

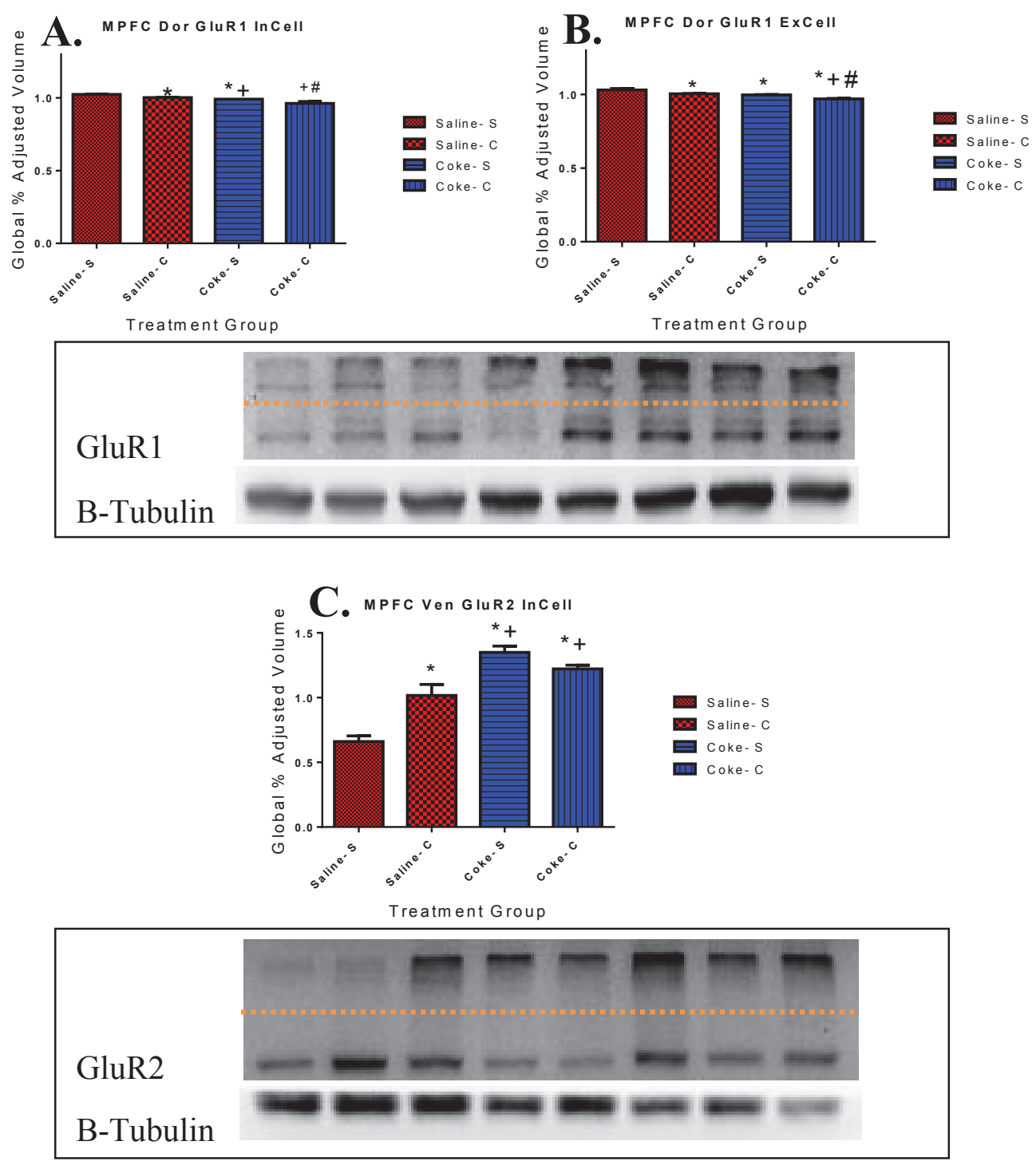

Figure 4-2. AMPA Receptor Changes at 2-Weeks.

Significant reductions GluR1 subunits are observed in the dorsal mPFC for both the intracellular (A, upper left) and extracellular (B, upper right) fractions, with the highest levels being observed in cocaine naïve controls, and the lowest levels in sensitized controls exposed on test day. For the intracellular $(\mathrm{C}$, bottom) fraction of GluR2 in the ventral $\mathrm{mPFC}$, surface expression is significantly elevated in all animals exposed to cocaine, with the highest levels found in sensitized animals, and although internalized receptor display this same trend, results are not significant. For all experimental groups, $n$ $=4$. For all panels, "**" indicates a significant change from cocaine naïve animals, and "+" indicates a significant change from acutely exposed saline animals, and "\#” indicates a significant change from sensitized animals that received saline on test day. Representative blots are located under each set of panels, with the dotted line representing the running/stacking gel interface that separates the upper surface fraction from the lower intercellular fraction. 

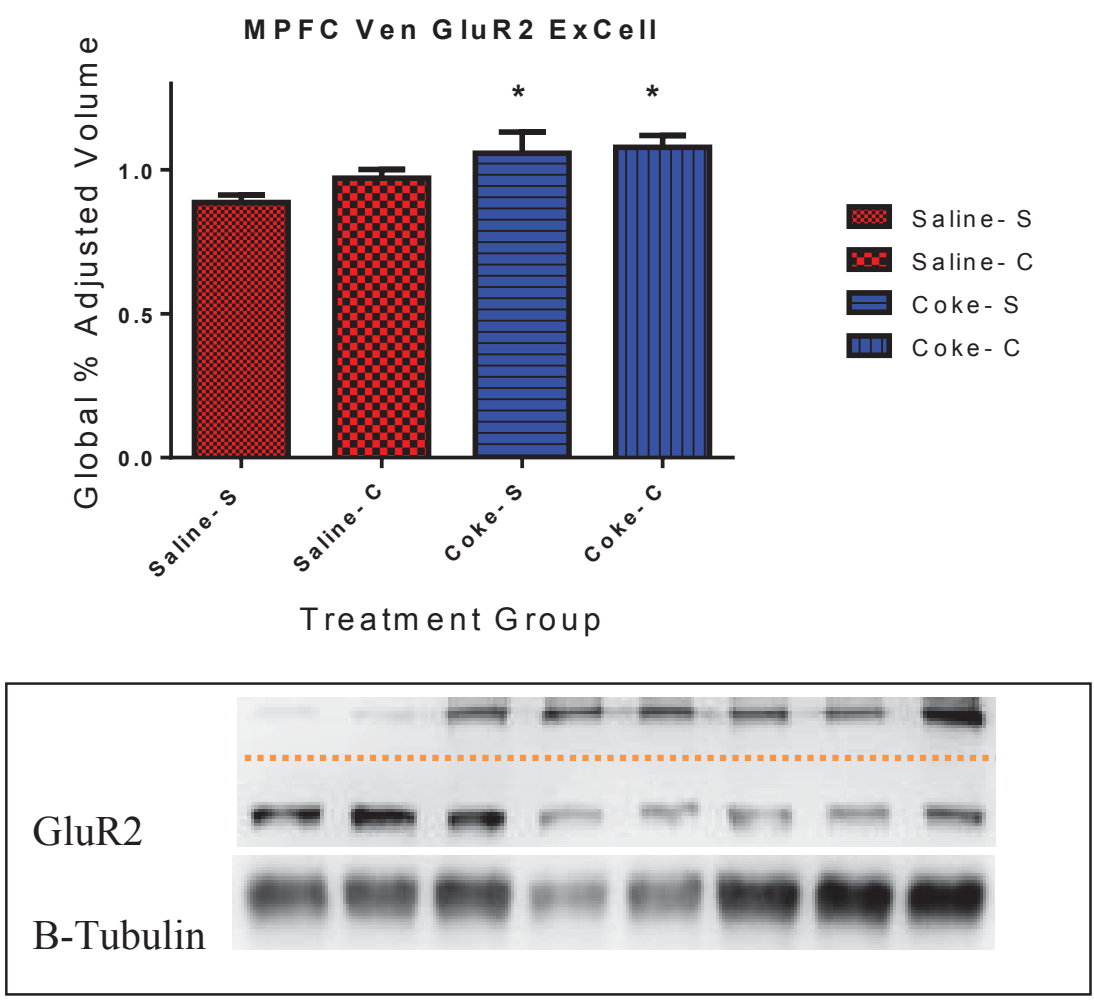

Figure 4-3. GluR2 Subunit Changes in the Ventral mPFC at 3-Weeks.

While a variety of iGluR modification were uncovered at the 2 -week time point, significant changes a week later appear to be restricted to the extracellular fraction of the ventral $\mathrm{mPFC}$, and consist of a significant increase in GluR2-containing AMPA receptors in sensitized animals regardless of challenge dose, over cocaine naïve controls. Note that “*” indicates a significant change from cocaine naive animals. Representative blot images are located under the panel, with the dotted line representing the running/stacking gel interface that separates the upper surface fraction from the lower intercellular fraction 


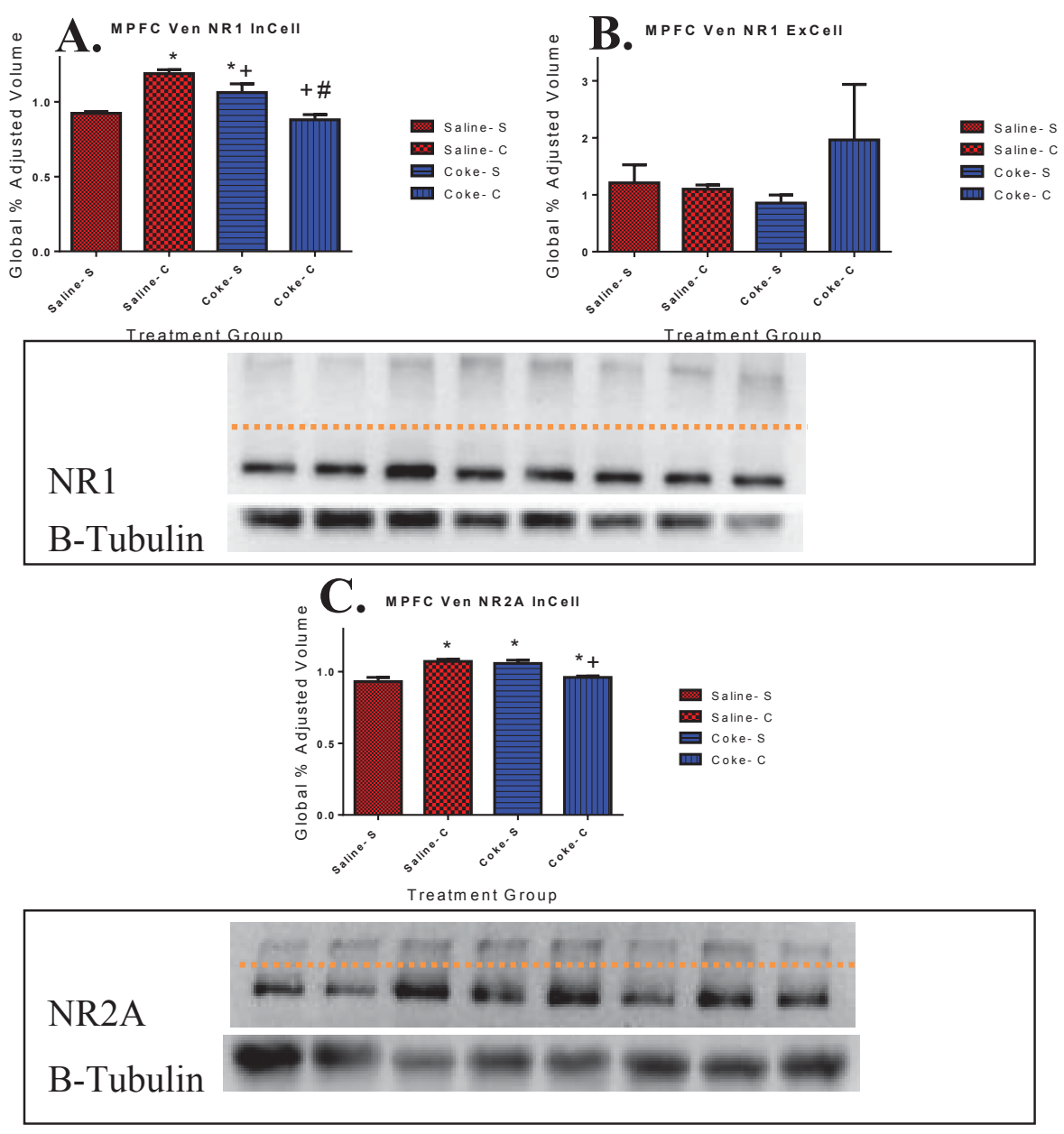

Figure 4-4. NR1 and NR2A Subunit Changes at 2-Weeks.

Significant alterations in internal NR1 subunits (A, upper left) for the ventral mPFC is noted, with a significant elevation over completely cocaine naïve controls observed in control animal exposed to a cocaine challenge on test day. However, NR1 levels decrease as cocaine exposure increases, with the lowest intracellular NR1 levels in cocaine sensitized animals exposure to cocaine on test day. Surface subunit expression in this region (B, upper right) follows oppositely trends, albeit none significantly. NR1 levels are in general decreased as cocaine exposure increases, yet the highest surface expression levels by far are observed in sensitized animals receiving drug on test day. The intracellular NR2A subunit fraction in the ventral $\mathrm{mPFC}(\mathrm{C}$, bottom) follows a similar patterns as in the dorsal mPFC. Protein levels increase in controls animals exposed to drug on test day over completely drug naïve animals. Yet, levels decrease in sensitized animals completely abstinent for 2 weeks, and even further in sensitized subjects receiving drug on test day. For all panels, "“*” indicates a significant change from cocaine naive animals, and "+" indicates a significant change from acutely exposed saline animals, and "\#" indicates a significant change from sensitized animals that received saline on test day. Representative blots are located under each set of panels, with the dotted line representing the running/stacking gel interface that separates the upper surface fraction from the lower intercellular fraction. 
for the external fraction, with a significant elevation observed when compared to cocaine naïve animals for the saline/cocaine group and the cocaine/saline group $(\mathrm{F}=10.94, \mathrm{df}=15$, $p=0.0009$, Figure 4-5). This result was also accompanied by a significant reduction in NR2A subunit when comparing to the saline/cocaine vs. cocaine/cocaine and cocaine/saline vs. cocaine/cocaine.

For the NR2B subunit in the dorsal mPFC, significant results were found for the external fraction only when compared the control rats and sensitized rats acutely exposed on test day $(\mathrm{F}=3.390, \mathrm{df}=15, \mathrm{p}=0.0539$, Figure 4-6A). Externally in the ventral $\mathrm{mPFC}$, NR2B levels are lowered in the cocaine/cocaine group when compared to the saline/cocaine and cocaine/saline groups $(\mathrm{F}=7.209, \mathrm{df}=15, \mathrm{p}=0.0050$, Figure 4-6C). Internally, results are significantly different when comparing saline/saline vs. cocaine/cocaine, saline/cocaine vs. cocaine/cocaine, and cocaine/saline vs. cocaine/cocaine $(\mathrm{F}=11.11, \mathrm{df}=15, \mathrm{p}=0.0009$, Figure 4-6B). At the 3 week withdrawal time point, significant changes appear to be restricted to the extracellular fraction of the ventral $\mathrm{mPFC}$, which displays a sensitization-specific subunit increase for the NR2B subunit $(\mathrm{F}=4.442, \mathrm{df}=23, \mathrm{p}=0.0151$, Figure 4-7). For NR2B, sensitized animals acutely exposed display elevated external levels when compared to control animals acutely exposed on test day as well as drug naïve controls.

\section{Discussion}

While initially reviewing the time-course for the whole receptor and subunit changes, the lack of receptor changes observed at 1-day post withdrawal fits with previous studies from our lab. This would indicate early transient changes in neurotransmitter release, and delayed changes in receptor function. However, glutamate receptor changes are capable of occurring on a scale ranging from minutes to hours [189].

These results could be influenced by our experimental design that specifies the sacrifice of animals at least 2 hours after exposure to a challenge dose of drug which would mask any very early receptor-centered effects of cocaine, effects of receptor phosphorylation that may precede removal or inclusion at the synapse [190], or that sensitization related changes may require more than 1 day post-withdrawal to begin to exert an effect on glutamate receptors. Also surprising was the finding that receptors in the prefrontal cortex in some control animals (completely cocaine naïve until given a challenge dose) begin to display acute prefrontal iGluR effects to drug, although it is not known if these effects are permanent or transient in nature. As sensitization changes continue to occur over the course of 3-weeks, more drastic changes in AMPA/NMDA receptor populations were expected as the time interval increased, yet a larger variety of changes appear to be observed at the 2-week withdrawal time-point instead of at the 3week time-point. Changes that are observed at 3-weeks post withdrawal affect sensitized animals similarly regardless of acute drug exposure which may possibly indicate an enhancement of long-term potentiation. This may be possible at 3-weeks, as LTP has been reported to occur in the $\mathrm{mPFC}$ of sensitized animals after 3-days of drug abstinence [191]. This study also suggested that these effects were caused by GABA(A) receptor 


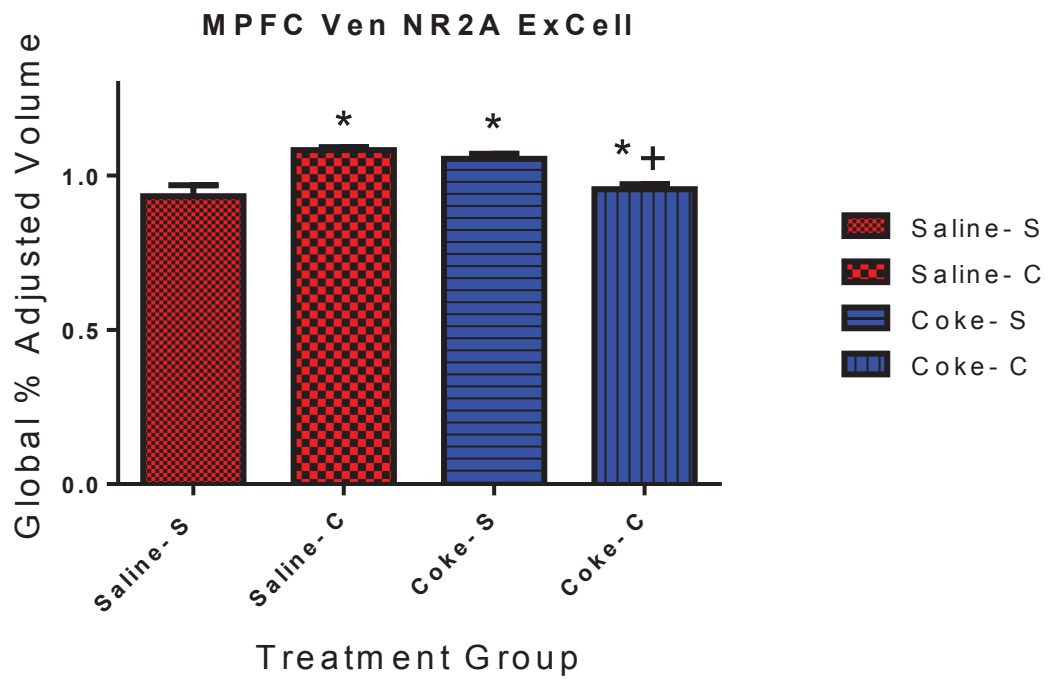

NR2A

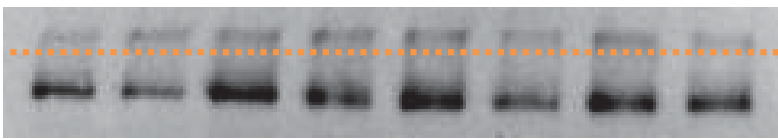

B-Tubulin

Figure 4-5. Surface NR2A Subunit Changes at 2-Weeks in the Ventral mPFC. Extracellular NR2A subunit fractions in the ventral mPFC follow similar patterns as in the dorsal $\mathrm{mPFC}$. Protein levels increase in controls animals exposed to drug on test day over completely drug naïve animals. Yet, levels decrease in sensitized animals completely abstinent for 2 weeks, and even further in sensitized subjects receiving drug on test day. Note that, "*" indicates a significant change from cocaine naive animals, and "+" indicates a significant change from acutely exposed saline animals. Representative blots are located under the panel, with the dotted line representing the running/stacking gel interface that separates the upper surface fraction from the lower intercellular fraction. 

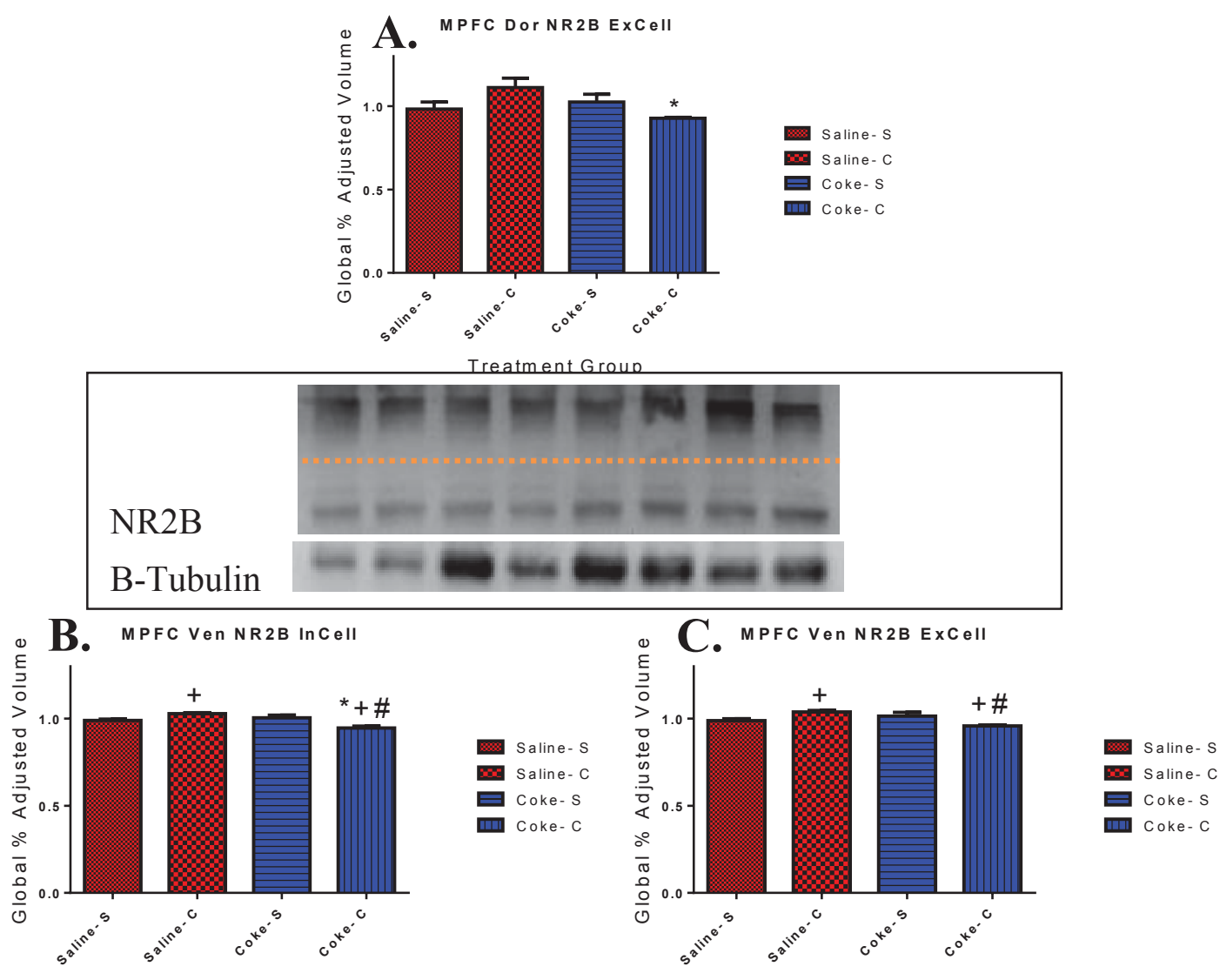

Treatment Group

Treatment Group

NR2B

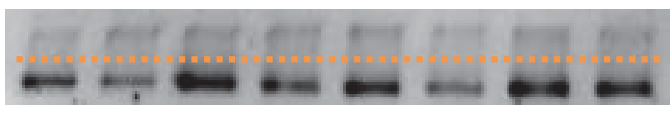

B-Tubulin

Figure 4-6. NR2B Subunit Changes at 2-Weeks.

For NR2B subunits, significant results were only obtained for the surface fraction of the dorsal mPFC (A, top), as sensitized animals drug exposed on test day showed significantly lower levels than other treatment groups. In contrast to the NR2B in the dorsal $\mathrm{mPFC}$, the ventral $\mathrm{mPFC}$ showed significant results in the surface (B, bottom left) and internal ( $\mathrm{C}$, bottom right), wherein significant results where only obtain for animals receiving drug on test day, an elevation in control animals and a reduction in sensitized animals over cocaine naïve controls. For all panels, “*” indicates a significant change from cocaine naive animals, "+" indicates a significant change from acutely exposed saline animals, and "\#" indicates a significant change from sensitized animals that received saline on test day. Representative blots are located under each set of panels, with the dotted line representing the running/stacking gel interface that separates the upper surface fraction from the lower intercellular fraction. 

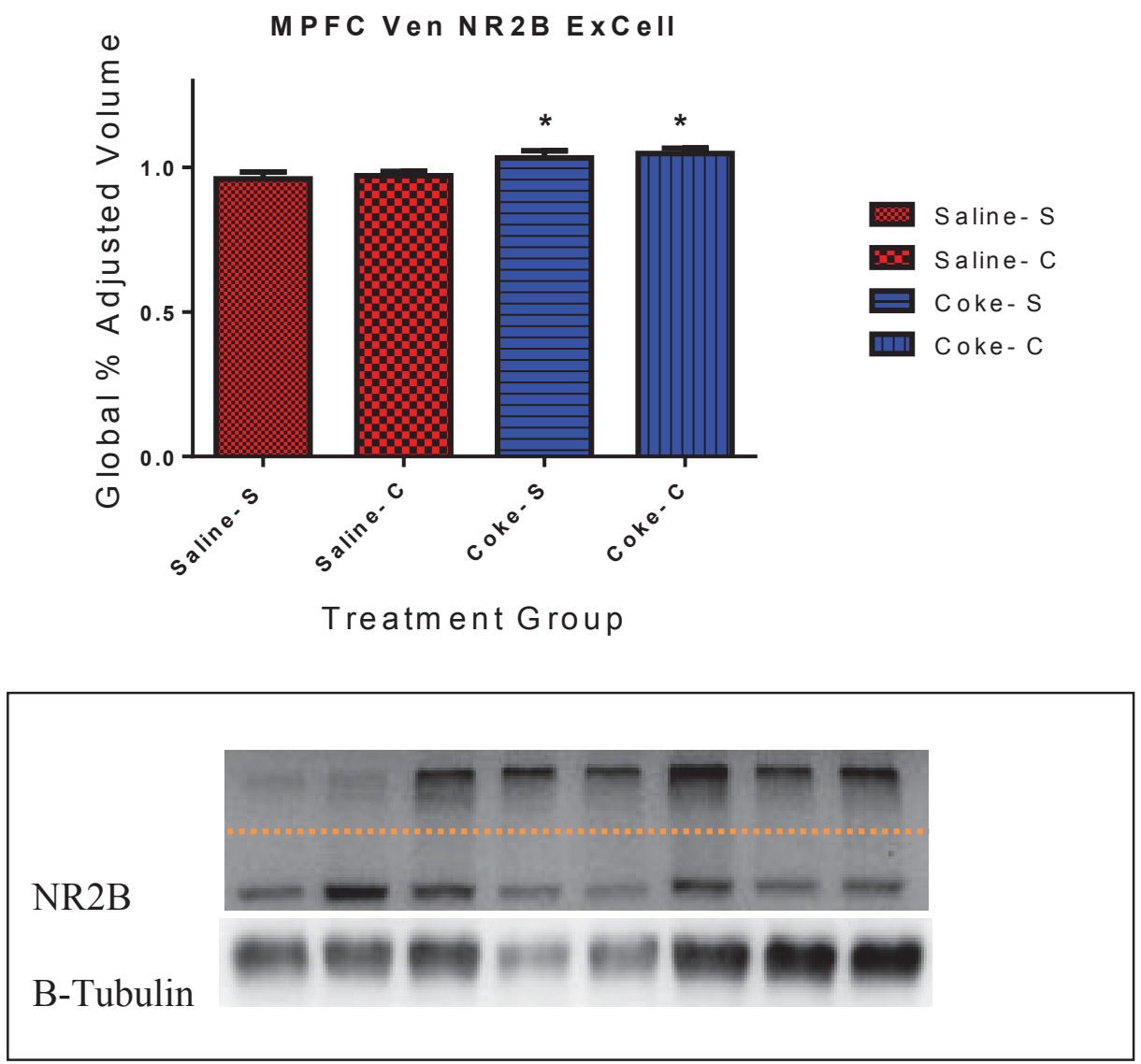

Figure 4-7. NR2B Subunit Changes in the Ventral mPFC at 3-Weeks.

While a variety of iGluR modification were uncovered at the 2-week time point, significant changes a week later appear to be restricted to the extracellular fraction of the ventral $\mathrm{mPFC}$, and consist of a significant increase in NR2B-containing NMDA receptors in sensitized animals regardless of challenge dose, over cocaine naïve controls. Note that “*” indicates a significant change from cocaine naive animals. Representative blot images are located under the panel, with the dotted line representing the running/stacking gel interface that separates the upper surface fractions from the lower intercellular fraction. 
blockade, which was later verified to occur in response to repeated cocaine exposure [192].

Despite these uncertainties, we may glean information on possible physiological functions through iGluR subunit alterations, albeit limited without corroborating electrophysiological studies. According to our data, at 1 day post-sensitization, changes are not present, yet by 2 -weeks changes are evident in the iGluR receptor population. At two weeks, animals exposed acutely to cocaine experience a reduction in GluR1 subunits in the dorsal $\mathrm{mPFC}$ that increases as cocaine exposure increases. Considering the concurrent increase in the ventral $\mathrm{mPFC}$, these events may represent a compensatory mechanism against excessive glutamate transmission. Even without a concurrent elevation of NMDA receptors in the dorsal $\mathrm{mPFC}$, this shift in the total ratio of iGluRs still has functional consequences, which may result in silent synapses, as NMDAreceptor input overpowers that of AMPA receptors. In contrast to the dorsal $\mathrm{mPFC}$, changes are present for NMDA receptor subunits the ventral $\mathrm{mPFC}$, and consist of intracellular down regulation of NR1 subunits, and a decrease in NR2A/B subunits. This may indicate less net expression of NMDA receptors, and increased contribution of AMPA receptors. In the "big picture", at 2-weeks, the documented changes may result in altered glutamate responses in the dorsal and ventral $\mathrm{mPFC}$, which includes GluR2 subunits to mute the enhanced AMPA currents. At 3-weeks, however, alterations in iGluRs are restricted to the ventral $\mathrm{mPFC}$. While receptors number is unchanged, there is an increase in surface GluR2 and NR2B subunits that is present in sensitized animals regardless of an acute cocaine challenge. This indicates that not only are these longerterm changes a fixture in sensitized animals, but that adaptations have occurred that reduce the excitability of AMPA receptors while increasing their impact due to decreased NMDAR contribution.

These data fit the known results of sensitization in the dorsal and ventral mPFC, in that the expression of behavioral sensitization can be induced by lesioning the dorsal mPFC [147] and reversed by inhibition of the ventral mPFC [193]. This lesioning of the dorsal $\mathrm{mPFC}$ may replicate consequences of this enhanced glutamate transmission in the dorsal mPFC. Excessive glutamate transmission is fatal to cortical pyramidal neurons [194], and mPFC lesioned animals find cocaine more reinforcing over sham controls [195] and display a sensitized response to amphetamine [196]. This is supported by the finding that ventral $\mathrm{mPFC}$ neurons (which contains direct glutamatergic projections to the Nacc) seem to be more responsive to glutamate in response to cocaine exposure [197]. Although the current results are promising, future experiments would be well served to conduct imaging to determine how the population of modulating mGluRs is altered, the exact layers that are affected by the receptor changes, and functionality how these detailed receptor changes affect and contribute to the expression of behavioral sensitization.

Our results in these two regions of the $\mathrm{mPFC}$ correspond to data published on the effect of $\mathrm{mPFC}$ cocaine exposure. In behavioral sensitization, cocaine induced changes occur initially in the dorsal $\mathrm{mPFC}$ when compared to those in the ventral $\mathrm{mPFC}$. If the dorsal $\mathrm{mPFC}$ is lesioned, this process is thwarted and behavioral sensitization cannot 
occur. If not prevented in some form, the effects of increased glutamatergic transmission will eventually permanently alter the ventral mPFC. While GABA agonists can prevent the effects of enhanced glutamatergic transmission in the ventral mPFC caused by acute cocaine and the initiation of behavioral sensitization, they cannot reverse changes already present. This would explain why baclofen is ineffective in preventing the expression of behavioral sensitization. Reversing the effects of sensitization in the ventral mPFC can prevent behavior associated with sensitization. Understanding these physiological changes could be a key step toward understanding the persistence of sensitization. The maintenance of a delicate balance of neurotransmitter regulation to the basal ganglia (i.e. the nucleus accumbens) is critical for healthy brain function. Any shifts in this balance tend to produce pathology [198]. Following this logic, we gain an appreciation of not only possibly why altered glutamate transmission has long been associated with psychiatric/nervous system disease [199, 200], with abnormalities indicated for many disorders including Huntington's Disease [201], schizophrenia [202], and bipolar disorder [203], and seemingly now cocaine addiction. These results not only advance the study of the underlying glutamatergic mechanisms responsible for cocaine sensitization, but may reveal clues of how glutamate is affected in all of these neurological diseases. 


\section{CHAPTER 5. CONCLUSION}

In summary, we conducted separate studies to examine the effects of cocaine sensitization. The first of these studies examined the effects of low-dose cocaine selfadministration in cocaine-sensitized animals, amphetamine-pretreated animals crosssensitized to cocaine, and non-sensitized sham-treated controls. A significantly higher number of psychostimulant-sensitized animals completed training when compared to non-sensitized sham-injected controls. While this has been reported to occur for higher doses of cocaine, this has not been reported to occur for a dose as low as 0.3 $\mathrm{mg} / \mathrm{kg} /$ infusion. For all of other parameters of self-administration examined, significant results were not found. Reasons for this are not certain, although factors that affect responding under self-administration may include the speed of which the cocaine reward was administered, a resistance of saline controls to acquire self-administration, and selfadministration occurring in a different environment than drug injections.

The second study examined the effects of acute cocaine exposure, cocaine sensitization and contingent cocaine exposure on SAPAP levels in the mesocorticolimbic circuit. Contingent cocaine exposure in sensitized animals generally results in increased SAPAP levels. These effects are transient, yet widespread and diverse in the regions and SAPAPs affected. Interestingly, non-contingently acutely exposed regions of the mesocorticolimbic circuit display a transient SAPAP decrease that generally recover to baseline levels $24 \mathrm{hrs}$ after exposure. In addition to these effects observed at shorter time points, at 3 weeks of abstinence all SAPAPs in the dorsal mPFC were significantly reduced in animals sensitized to cocaine and acutely exposed on test day. For this time point in the Nacc core, all SAPAPs were decreased in a singular, consistent manner, with significantly lower levels observed in response to an acute dose on test day in sensitized animals. Moreover, for all withdrawal time points, while nucleus accumbens shell, dorsal striatum, substantia nigra, and ventral tegmental area were examined for all 4 SAPAPs, none of these areas displayed statistically significant differences. Differing from this pattern, at 2 weeks post-withdrawal in the dorsal mPFC SAPAPs 3 and 4 that decreased in cocaine-sensitized animals exposed to cocaine on test day. For our self-administration experiment in this study, SAPAP levels were altered in test animals responding under FR1 for cocaine rewards. SAPAP protein levels for test animals were observed to be significantly higher for all SAPAPs in the dorsal mPFC, SAPAP3 in the ventral mPFC, and SAPAPs 2 and 4 in the Nacc core.

The third and final study examined the effects of cocaine sensitization and acute cocaine exposure on the AMPA/NMDA receptor population in the medial prefrontal cortex. Significant receptor subunit changes were not found for animals abstinent from cocaine for 1-day, which confirmed data for other studies from our research group. At 2 weeks of cocaine abstinence, animals exposed acutely to cocaine experienced a reduction in GluR1 subunits in the dorsal $\mathrm{mPFC}$ in a fashion that increases as cocaine exposure increases, with a concurrent increase in the ventral mPFC. Also at 2-weeks in the ventral mPFC, we observe a decrease in all intracellular NMDAR subunits. This indicates that there is less net expression of NMDA receptors and increased contribution of AMPA 
receptors in the ventral mPFC. Subunit changes that are observed at 3 weeks post withdrawal affect sensitized animals similarly. This occurs regardless of acute drug exposure, which may possibly indicate an enhancement of long-term potentiation (LTP). This is unsurprising, as an enhancement of LTP is reported to occur in the mPFC of sensitized animals after only 3 days of drug abstinence. These changes are restricted to the extracellular fraction of the ventral $\mathrm{mPFC}$, and included sensitization-specific subunit increase for the NR2B and GluR2 subunits. For these subunits, sensitized animals acutely exposed display elevated external levels when compared to previously cocaine naïve animals acutely exposed on test day.

In comparing independently-gathered results to published studies, seemingly disparate studies can fit together in a cohesive manner. An example of these in observed for our prefrontal iGluR and SAPAP studies. In sensitized animals, normal function can be restored to the glutamatergically-overactive dorsal striatum by the introduction of AMPA antagonist [204]. This would fit our findings for the SAPAP studies, as the NMDA/AMPA receptor ratio is vital to proper synaptic functioning [205]. This lack of counter-input to AMPA receptors would be explained by decreased SAPAP levels, perhaps because receptors that are not anchored to the PSD tend to be removed from the PSD [206]. Yet, this may also occur because these NMDA receptors are not clustered together, presently a functionally inefficient arrangement that impairs functions. In response to cocaine self-administration, the finding that all SAPAPs in the dorsal mPFC and dendritic SAPAP3 in the ventral $\mathrm{mPFC}$ are altered may reflect the unequal roles of these regions in the sensitization process. In cocaine-naïve animals such as the test animals, the dorsal mPFC is implicated in the effects of acute effects of cocaine exposure, with effects developing later in the ventral $\mathrm{mPFC}$ of chronically-exposed animals. Although we only examined protein changes, sensitized animals may experience misregulation at a deeper level. Our results here have been partially confirmed by other researchers with the discovery that repeated cocaine exposure is found to increase prefrontal GluR2 mRNA after 3 weeks of abstinence [207]. In agreement with a wider body a literature, this study also shows that in response to repeated cocaine mGluR5 levels are altered [207]. These same alterations may even extend beyond the context of drug use and addiction, as similar changes in SAPAPs, AMPARs, NMDARs, and mGluR5 are implicated in neurodevelopmental disorders that cause social dysfunction [208].

Suggestions for future self-administration experiments include using faster infusion pumps, and to sensitize animals in the operant testing environment. For future experiments involving the study of SAPAPs, a detailed account of location specific changes in the regions examined, as various cell types/layers differ in their mRNA SAPAP composition [172], and mechanisms regulating their expression patterns in cocaine naïve versus sensitized animals will help to uncover the exact purpose of these functionally mysterious family of proteins and how they are affected by cocaine sensitization. Although we suggest that SAPAP depletion could be responsible for compulsive symptoms, we have not tested for compulsivity in sensitized animals. Unexpectedly, sensitized rats lesioned with ibotenic acid in the mPFC, while still displaying locomotor sensitization, also displayed increased grooming behavior [156], 
possibly implicating both reduced prefrontal function in this particular compulsive behavior. Although it has been observed that a small number of our sensitized animals display a "bald" area on their snout, a simple visual survey of sensitized animals could yield quantitative data on frequency of bald areas, and if they are linked to compulsive grooming. While functional properties of glutamate receptors in the $\mathrm{mPFC}$ were not investigated in our studies, much is known about how these changes influence receptor function. Along with experiments planned to experimentally alter SAPAP levels, these receptors may deserve close attention, especially considering that the activity of NMDA receptors are regulated through phosphorylation by SAPAPs and that mGluRs, while not directly altering synaptic current, contribute greatly to the responses of iGluRs. Moreover, both classes of receptor are vital to the progression of research involving SAPAPs. Not only are SAPAPs necessary to link NMDARs to the underlying cytoskeleton [85], but recent studies have implicated both SAPAP3 and mGluR5 as prime mediators in synaptic silencing [141]. SAPAP3 has also been implicated in endocannabinoid-related synaptic plasticity [209].

While studied separately for the sake of simplicity, the changes we report all occur in concert in response to cocaine. Considering this, there is no reason to assume that our self-administration subject animal that displayed the greatest preference for drug and greatest resistance to extinction also would not have also been found to have the lowest levels of SAPAP3 and the most drastically altered iGluRs. Evidence of this impingement may be found in our preliminary experiments. For one such experiment, we selected animals that were age-matched cage mates. These rats were sensitized under identical conditions. Rats were randomly chosen to either be probed for SAPAP or iGluR changes after a 3-week abstinence period. The animals chosen for the SAPAP studies displayed a decrease in prefrontal SAPAP3 levels. Additionally, those chosen for iGluR studies displayed an increase in surface NR1 subunit levels. Both of these findings correspond directly with cocaine exposure (Figure 5-1). Interactions between SAPAPs and iGluRs may also in part explain the enhanced acquisition of reinforcement training in sensitized animals. As a possible mechanistic explanation for our data, if SAPAPs are absent from the PSD, several self-perpetuating changes may occur. Firstly, the degradation or inactivation of SAPAP results in increased dendritic outgrowth similar to that observed in MSNs in the nucleus accumbens. If SAPAPs proteins are reduced, this would explain the increased dopaminergic tone in the medial prefrontal cortex. This increase in dopamine would account for the increased glutamate release not only in this region, but also in areas where these neurons project (including the nucleus accumbens). In addition, reciprocating glutamatergic release onto the Nacc has an increased effect, as SAPAP degradation results in increased areas of input due to increased dendrites. Combined with the consideration that SAPAPs serve to activate NMDA receptors through their guanylate kinase domains, absence of these molecules may explain the greater contribution of excitatory currents in published studies. This may also help to our results of increased NMDA receptors, as these cortical neurons may compensate for lack of NMDA receptor input by expressing more NMDA receptors. Perhaps due to this putative increased contribution of excitatory AMPA currents, cortical neurons may be hypo-functional, a possible cause of increased operant responding in sensitized animals due to decreased executive function 


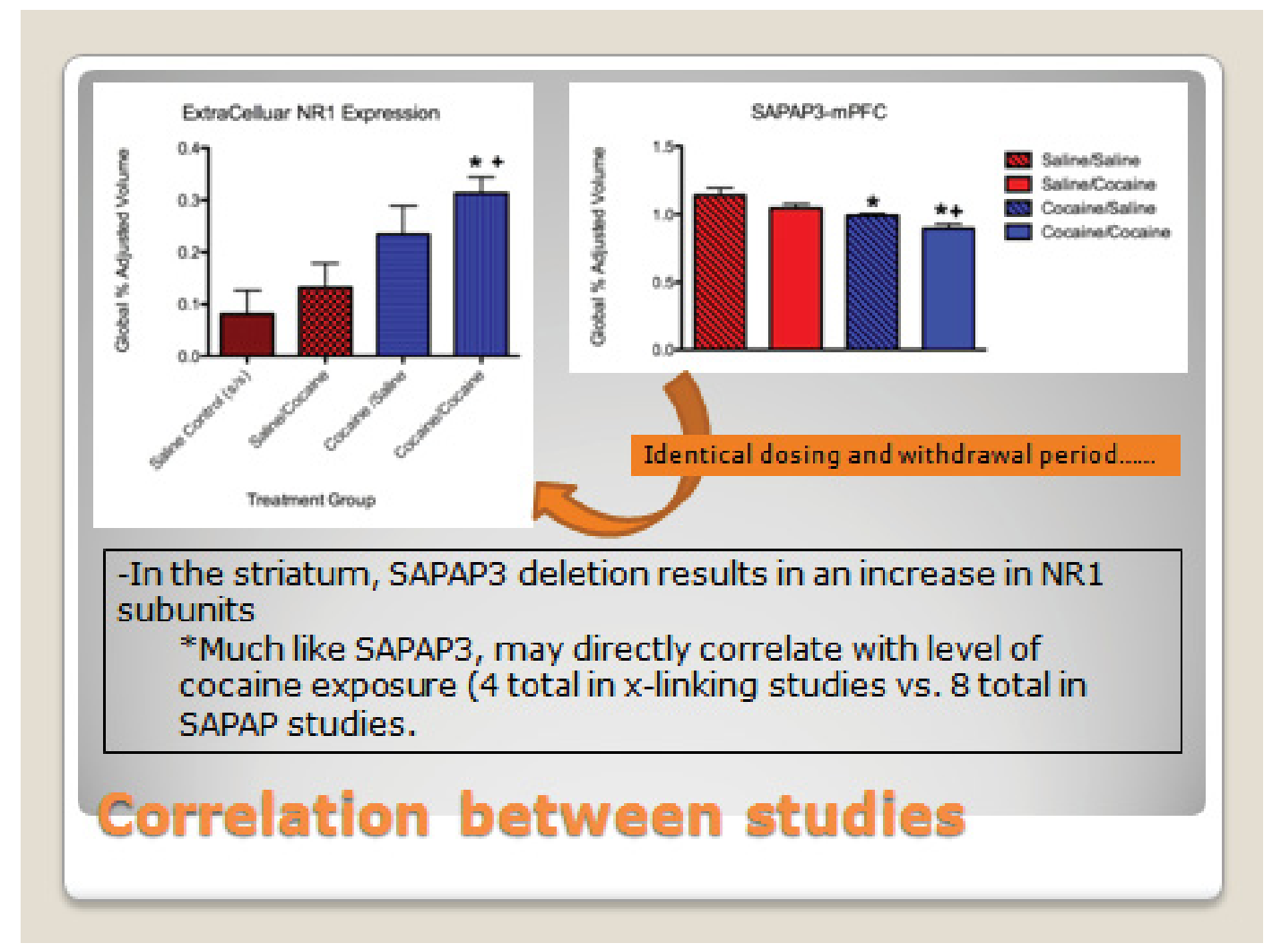

Figure 5-1. Possible Correlation Between SAPAP and AMPA/NMDA Receptor Studies.

In rats sensitized at exactly the same time in the exactly same manner, animals selected for SAPAP studies displayed a decrease in prefrontal SAPAP3 levels, while those chosen for iGluR studies displayed an increase in surface NR1 subunit levels, both which corresponding directly with cocaine exposure. Note that "*” indicates a significant change from yoked animals, and "+" indicates a significant change from acutely exposed saline animals. 


\section{LIST OF REFERENCES}

1. Bernfeld, S., Freud's studies on cocaine, 1884-1887. J Am Psychoanal Assoc, 1953. 1(4): p. 581-613.

2. Classics revisited. Uber Coca. By Sigmund Freud. J Subst Abuse Treat, revisited 1984; original 1884. 1(3): p. 206-17.

3. Elsohly, M.A., et al., Study of the concentration of the herbicide (2,4dichlorophenoxy)-acetic acid in coca leaves and paste obtained from plants treated with this herbicide. Bull Narc, 1984. 36(2): p. 65-77.

4. Di Maio, V.J. and J.C. Garriott, Four deaths due to intravenous injection of cocaine. Forensic Sci Int, 1978. 12(2): p. 119-25.

5. Wetli, C.V. and D.A. Fishbain, Cocaine-induced psychosis and sudden death in recreational cocaine users. J Forensic Sci, 1985. 30(3): p. 873-80.

6. Luborsky, L., et al., To use or to refuse cocaine--the deciding factors. J Subst Abuse, 1995. 7(3): p. 293-310.

7. Ahmed, S.H., The science of making drug-addicted animals. Neuroscience, 2012. 211: p. 107-25.

8. Comings, D.E., et al., The dopamine D2 receptor gene: a genetic risk factor in substance abuse. Drug Alcohol Depend, 1994. 34(3): p. 175-80.

9. Banducci, A.N., et al., The relationship between child abuse and negative outcomes among substance users: psychopathology, health, and comorbidities. Addict Behav, 2014. 39(10): p. 1522-7.

10. Picetti, R., et al., Addictions and stress: clues for cocaine pharmacotherapies. Curr Pharm Des, 2013. 19(40): p. 7065-80.

11. Shuster, L., G. Yu, and A. Bates, Sensitization to cocaine stimulation in mice. Psychopharmacology (Berl), 1977. 52(2): p. 185-90.

12. Cocaine Hallucinations. Science, 1889. 14(354): p. 332.

13. W. Golden Mortimer, M.D., Peru History of Coca: "The Divine Plant of the Incas", with an introductory account of the Incas, and of the Andean Indians of to-day 1901, New York, New York: J. H. Vail \& Company. 616. 
14. Rothman, R.B., et al., Lack of evidence for context-dependent cocaine-induced sensitization in humans: preliminary studies. Pharmacol Biochem Behav, 1994. 49(3): p. 583-8.

15. Perez-Reyes, M., et al., Clinical effects of daily methamphetamine administration. Clin Neuropharmacol, 1991. 14(4): p. 352-8.

16. Strakowski, S.M., et al., Enhanced response to repeated d-amphetamine challenge: Evidence for behavioral sensitization in humans. Biol Psychiatry, 1996. 40(9): p. 872-880.

17. Rosse, R.B., et al., Transient compulsive foraging behavior associated with crack cocaine use. Am J Psychiatry, 1993. 150(1): p. 155-6.

18. Rosse, R.B., et al., The relationship between cocaine-induced paranoia and compulsive foraging: a preliminary report. Addiction, 1994. 89(9): p. 1097-104.

19. Post, R.M. and H. Rose, Increasing effects of repetitive cocaine administration in the rat. Nature, 1976. 260(5553): p. 731-2.

20. Saunders, B.T., et al., A cocaine context renews drug seeking preferentially in a subset of individuals. Neuropsychopharmacology, 2014. 39(12): p. 2816-23.

21. Sutton, M.A., D.A. Karanian, and D.W. Self, Factors that determine a propensity for cocaine-seeking behavior during abstinence in rats.

Neuropsychopharmacology, 2000. 22(6): p. 626-41.

22. Mandt, B.H., et al., Cocaine dose and self-administration history, but not initial cocaine locomotor responsiveness, affects sensitization to the motivational effects of cocaine in rats. J Pharmacol Exp Ther, 2012. 342(1): p. 214-21.

23. Goeders, N.E. and J.E. Smith, Reinforcing properties of cocaine in the medical prefrontal cortex: primary action on presynaptic dopaminergic terminals. Pharmacol Biochem Behav, 1986. 25(1): p. 191-9.

24. Robinson, T.E. and B. Kolb, Alterations in the morphology of dendrites and dendritic spines in the nucleus accumbens and prefrontal cortex following repeated treatment with amphetamine or cocaine. Eur J Neurosci, 1999. 11(5): p. 1598-604.

25. Paulson, P.E., D.M. Camp, and T.E. Robinson, Time course of transient behavioral depression and persistent behavioral sensitization in relation to regional brain monoamine concentrations during amphetamine withdrawal in rats. Psychopharmacology (Berl), 1991. 103(4): p. 480-92. 
26. Jones, D.C. and D. Kimeldorf, Lifespan measurements in the male rat, 1963, DTIC Document.

27. Crow, T.J., Enhancement of coaine of intra-cranial self-stimulation in the rat. Life Sci, 1970. 9(7): p. 375-81.

28. Lippi, G., M. Plebani, and G. Cervellin, Cocaine in acute myocardial infarction. Adv Clin Chem, 2010. 51: p. 53-70.

29. Bunn, W.H. and A.J. Giannini, Cardiovascular complications of cocaine abuse. Am Fam Physician, 1992. 46(3): p. 769-73.

30. Benzaquen, B.S., V. Cohen, and M.J. Eisenberg, Effects of cocaine on the coronary arteries. Am Heart J, 2001. 142(3): p. 402-10.

31. Manetti, L., et al., Effects of cocaine on the hypothalamic-pituitary-adrenal axis. J Endocrinol Invest, 2014.

32. Carroll, F.I., et al., Synthesis, monoamine transporter binding properties, and behavioral pharmacology of a series of 3beta-(substituted phenyl)-2beta-(3'substituted isoxazol-5-yl)tropanes. J Med Chem, 2004. 47(2): p. 296-302.

33. Scherman, D., Dihydrotetrabenazine binding and monoamine uptake in mouse brain regions. J Neurochem, 1986. 47(2): p. 331-9.

34. Heard, K., R. Palmer, and N.R. Zahniser, Mechanisms of acute cocaine toxicity. Open Pharmacol J, 2008. 2(9): p. 70-78.

35. Fleckenstein, A.E., et al., Differential effects of psychostimulants and related agents on dopaminergic and serotonergic transporter function. Eur J Pharmacol, 1999. 382(1): p. 45-9.

36. Iversen, L.L., Inhibition of Noradrenaline Uptake by Drugs. J Pharm Pharmacol, 1965. 17: p. $62-4$.

37. White, F.J., Electrophysiological basis of the reinforcing effects of cocaine. Behav Pharmacol, 1990. 1(4): p. 303-315.

38. Kuhar, M.J., M.C. Ritz, and J.W. Boja, The dopamine hypothesis of the reinforcing properties of cocaine. Trends Neurosci, 1991. 14(7): p. 299-302.

39. Ritz, M.C., et al., Cocaine receptors on dopamine transporters are related to selfadministration of cocaine. Science, 1987. 237(4819): p. 1219-23. 
40. Lile, J.A., et al., The reinforcing efficacy of the dopamine reuptake inhibitor 2beta-propanoyl-3beta-(4-tolyl)-tropane (PTT) as measured by a progressiveratio schedule and a choice procedure in rhesus monkeys. J Pharmacol Exp Ther, 2002. 303(2): p. 640-8.

41. Downs, A.W.E., N.B., The Effect of Repeated Cocaine on the Rat. J. Pharmacol. Exp. Ther., 1932(46): p. 3.

42. Lett, B.T., Repeated exposures intensify rather than diminish the rewarding effects of amphetamine, morphine, and cocaine. Psychopharmacology (Berl), 1989. 98(3): p. 357-62.

43. Sorg, B.A. and C. Ulibarri, Application of a protein synthesis inhibitor into the ventral tegmental area, but not the nucleus accumbens, prevents behavioral sensitization to cocaine. Synapse, 1995. 20(3): p. 217-24.

44. Steketee, J.D., Intra-A10 injection of H7 blocks the development of sensitization to cocaine. Neuroreport, 1994. 6(1): p. 69-72.

45. Kalivas, P.W., Interactions between dopamine and excitatory amino acids in behavioral sensitization to psychostimulants. Drug Alcohol Depend, 1995. 37(2): p. 95-100.

46. Steketee, J.D., Neurotransmitter systems of the medial prefrontal cortex: potential role in sensitization to psychostimulants. Brain Res Brain Res Rev, 2003. 41(2-3): p. 203-28.

47. Otani, S., et al., Dopamine facilitates long-term depression of glutamatergic transmission in rat prefrontal cortex. Neuroscience, 1998. 85(3): p. 669-76.

48. Galloway, M.P., Regulation of dopamine and serotonin synthesis by acute administration of cocaine. Synapse, 1990. 6(1): p. 63-72.

49. Rasakham, K., et al., Synapse density and dendritic complexity are reduced in the prefrontal cortex following seven days of forced abstinence from cocaine selfadministration. PLoS One, 2014. 9(7): p. e102524.

50. Allen, C.P. and F. Leri, Perseveration in the presence of punishment: the effects of chronic cocaine exposure and lesions to the prefrontal cortex. Behav Brain Res, 2014. 261: p. 185-92.

51. Baca, S.M., et al., Effects of prefrontal cortical lesions on neuropeptide and dopamine receptor gene expression in the striatum-accumbens complex. Brain Res, 1998. 797(1): p. 55-64. 
52. Song, S.S., et al., Optogenetics reveals a role for accumbal medium spiny neurons expressing dopamine D2 receptors in cocaine-induced behavioral sensitization. Front Behav Neurosci, 2014. 8: p. 336.

53. Bolla, K., et al., Prefrontal cortical dysfunction in abstinent cocaine abusers. J Neuropsychiatry Clin Neurosci, 2004. 16(4): p. 456-64.

54. McLennan, H., Receptors for the excitatory amino acids in the mammalian central nervous system. Prog Neurobiol, 1983. 20(3-4): p. 251-71.

55. Jaspers, R.M., T.J. de Vries, and A.R. Cools, Enhancement in switching motor patterns following local application of the glutamate agonist AMPA into the cat caudate nucleus. Behav Brain Res, 1990. 37(3): p. 237-46.

56. Honore, T., Excitatory amino acid receptor subtypes and specific antagonists. Med Res Rev, 1989. 9(1): p. 1-23.

57. Hansen, J.J., et al., Excitatory amino acid agonists. Enzymic resolution, X-ray structure, and enantioselective activities of $(R)$ - and (S)-bromohomoibotenic acid. J Med Chem, 1989. 32(10): p. 2254-60.

58. Rossmann, M., et al., Subunit-selective N-terminal domain associations organize the formation of AMPA receptor heteromers. EMBO J, 2011.30(5): p. 959-71.

59. Connaughton, V., Glutamate and Glutamate Receptors in the Vertebrate Retina., in Webvision: The Organization of the Retina and Visual System [Internet], F.E. Kolb H, Nelson R, Editor 2005 May 1 [Updated 2007 May 7], University of Utah Health Sciences Center: Salt Lak City, UT.

60. Muller, D., M. Joly, and G. Lynch, Contributions of quisqualate and NMDA receptors to the induction and expression of LTP. Science, 1988. 242(4886): $\mathrm{p}$. 1694-7.

61. Bliss, T.V. and G.L. Collingridge, A synaptic model of memory: long-term potentiation in the hippocampus. Nature, 1993. 361(6407): p. 31-9.

62. Cummings, J.A., et al., Ca2+ signaling requirements for long-term depression in the hippocampus. Neuron, 1996. 16(4): p. 825-33.

63. Zucker, R.S., Calcium- and activity-dependent synaptic plasticity. Curr Opin Neurobiol, 1999. 9(3): p. 305-13.

64. Choi, D.W., Calcium: still center-stage in hypoxic-ischemic neuronal death. Trends Neurosci, 1995. 18(2): p. 58-60. 
65. Moriyoshi, K., et al., Molecular cloning and characterization of the rat NMDA receptor. Nature, 1991. 354(6348): p. 31-7.

66. Kornau, H.C., et al., Domain interaction between NMDA receptor subunits and the postsynaptic density protein PSD-95. Science, 1995. 269(5231): p. 1737-40.

67. Sobolevsky, A.I., M.P. Rosconi, and E. Gouaux, X-ray structure, symmetry and mechanism of an AMPA-subtype glutamate receptor. Nature, 2009. 462(7274): p. 745-56.

68. Mori, H. and M. Mishina, Structure and function of the NMDA receptor channel. Neuropharmacology, 1995. 34(10): p. 1219-37.

69. Zhang, X.F., et al., Increased responsiveness of ventral tegmental area dopamine neurons to glutamate after repeated administration of cocaine or amphetamine is transient and selectively involves AMPA receptors. J Pharmacol Exp Ther, 1997. 281(2): p. 699-706.

70. Wang, Y.H., et al., Characterization of NMDA receptor subunit-specific antibodies: distribution of $N R 2 A$ and $N R 2 B$ receptor subunits in rat brain and ontogenic profile in the cerebellum. J Neurochem, 1995. 65(1): p. 176-83.

71. Licata, S.C. and P.F. Renshaw, Neurochemistry of Drug Action: Insights from Proton Magnetic Resonance Spectroscopic Imaging And Their Relevance to Addiction. Ann N Y Acad Sci, 2010. 1187: p. 148-171.

72. Kim, M.J., et al., Differential Roles of NR2A- and NR2B-Containing NMDA Receptors in Ras-ERK Signaling and AMPA Receptor Trafficking. Neuron, 2005. 46(5): p. 745-760.

73. Scholz, R., et al., AMPA Receptor Signaling through BRAG2 and Arf6 Critical for Long-Term Synaptic Depression. Neuron, 2010. 66(5): p. 768-780.

74. Snyder, G.L., et al., Regulation of phosphorylation of the GluR1 AMPA receptor in the neostriatum by dopamine and psychostimulants in vivo. J Neurosci, 2000. 20(12): p. 4480-8.

75. Gourley, S.L., et al., Cytoskeletal determinants of stimulus-response habits. J Neurosci, 2013. 33(29): p. 11811-6.

76. Robinson, T.E. and B. Kolb, Structural plasticity associated with exposure to drugs of abuse. Neuropharmacology, 2004. 47 Suppl 1: p. 33-46.

77. Wei, Z., et al., Subunit-Specific Regulation of N-Methyl-D-aspartate (NMDA) Receptor Trafficking by SAP102 Splice Variants. J Biol Chem, 2015. 
78. Small, G.W. and J.J. Purcell, Trazodone and cocaine abuse. Arch Gen Psychiatry, 1985. 42(5): p. 524.

79. Khouzam, H.R., et al., Treatment of crack-cocaine-induced compulsive behavior with trazodone. J Subst Abuse Treat, 1995. 12(2): p. 85-8.

80. Gorski, T.T., Managing Cocaine Craving. 1996: Hazelden Publishing. 28.

81. Fals-Stewart, W. and J. Schafer, The treatment of substance abusers diagnosed with obsessive-compulsive disorder: an outcome study. J Subst Abuse Treat, 1992. 9(4): p. 365-70.

82. Hata, Y. and Y. Takai, Roles of postsynaptic density-95/synapse-associated protein 90 and its interacting proteins in the organization of synapses. Cell Mol Life Sci, 1999. 56(5-6): p. 461-72.

83. Ehlers, M.D., et al., Synaptic targeting of glutamate receptors. Curr Opin Cell Biol, 1996. 8(4): p. 484-9.

84. Gomperts, S.N., Clustering membrane proteins: It's all coming together with the PSD-95/SAP90 protein family. Cell, 1996. 84(5): p. 659-62.

85. Takeuchi, M., et al., SAPAPs. A family of PSD-95/SAP90-associated proteins localized at postsynaptic density. J Biol Chem, 1997. 272(18): p. 11943-51.

86. Naisbitt, S., et al., Shank, a novel family of postsynaptic density proteins that binds to the NMDA receptor/PSD-95/GKAP complex and cortactin. Neuron, 1999. 23(3): p. 569-82.

87. Yamada, Y., et al., Activation of channel activity of the NMDA receptor-PSD-95 complex by guanylate kinase-associated protein (GKAP). FEBS Lett, 1999. 458(3): p. 295-8.

88. Moutin, E., et al., GKAP-DLC2 interaction organizes the postsynaptic scaffold complex to enhance synaptic NMDA receptor activity. J Cell Sci, 2012. 125(Pt 8): p. 2030-40.

89. Welch, J.M., et al., Cortico-striatal synaptic defects and OCD-like behaviours in Sapap3-mutant mice. Nature, 2007. 448(7156): p. 894-900.

90. Morton, W.A., Cocaine and Psychiatric Symptoms. Prim Care Companion J Clin Psychiatry, 1999. 1(4): p. 109-113.

91. Anonymous, N., Nacotics Anonymous. 1987, New York City, New York: World Service Office, Inc. 290. 
92. Kucera, D., Everything I Never Wanted to Be: A Memoir of Alcoholism and Addiction, Faith and Family, Hope and Humor. 2010, Downers Grove, IL, USA: Dream of Things. 216.

93. Di Chiara, G., et al., Drugs of abuse: biochemical surrogates of specific aspects of natural reward? Biochem Soc Symp, 1993. 59: p. 65-81.

94. Liu, Y., D. Morgan, and D.C. Roberts, Cross-sensitization of the reinforcing effects of cocaine and amphetamine in rats. Psychopharmacology (Berl), 2007. 195(3): p. 369-75.

95. Suto, N., et al., Previous exposure to psychostimulants enhances the reinstatement of cocaine seeking by nucleus accumbens AMPA. Neuropsychopharmacology, 2004. 29(12): p. 2149-59.

96. Ferrario, C.R. and T.E. Robinson, Amphetamine pretreatment accelerates the subsequent escalation of cocaine self-administration behavior. Eur Neuropsychopharmacol, 2007. 17(5): p. 352-7.

97. Yehuda, S. and R.J. Wurtman, Release of brain dopamine as the probable mechanism for the hypothermic effect of D-amphetamine. Nature, 1972. 240(5382): p. 477-8.

98. Jatlow, P., et al., Cocaethylene: a neuropharmacologically active metabolite associated with concurrent cocaine-ethanol ingestion. Life Sci, 1991. 48(18): p. 1787-94.

99. Hearn, W.L., et al., Cocaethylene is more potent than cocaine in mediating lethality. Pharmacol Biochem Behav, 1991. 39(2): p. 531-3.

100. Hearn, W.L., et al., Cocaethylene: a unique cocaine metabolite displays high affinity for the dopamine transporter. J Neurochem, 1991. 56(2): p. 698-701.

101. Wilder, R.L., et al., Susceptibility to autoimmune disease and drug addiction in inbred rats. Are there mechanistic factors in common related to abnormalities in hypothalamic-pituitary-adrenal axis and stress response function? Ann N Y Acad Sci, 2000. 917: p. 784-96.

102. Shaham, Y., S. Erb, and J. Stewart, Stress-induced relapse to heroin and cocaine seeking in rats: a review. Brain Res Brain Res Rev, 2000. 33(1): p. 13-33.

103. Morrow, B.A., et al., (S)-(-)-HA-966, a gamma-hydroxybutyrate-like agent, prevents enhanced mesocorticolimbic dopamine metabolism and behavioral correlates of restraint stress, conditioned fear and cocaine sensitization. $\mathrm{J}$ Pharmacol Exp Ther, 1997. 283(2): p. 712-21. 
104. Covington, H.E., 3rd and K.A. Miczek, Repeated social-defeat stress, cocaine or morphine. Effects on behavioral sensitization and intravenous cocaine selfadministration "binges". Psychopharmacology (Berl), 2001. 158(4): p. 388-98.

105. Ogbonmwan, Y.E., et al., The effects of post-extinction exercise on cocaineprimed and stress-induced reinstatement of cocaine seeking in rats. Psychopharmacology (Berl), 2014.

106. Phillips, A.G. and P. Di Ciano, Behavioral sensitization is induced by intravenous self-administration of cocaine by rats. Psychopharmacology (Berl), 1996. 124(3): p. 279-81.

107. Schenk, S. and B. Partridge, Sensitization to cocaine's reinforcing effects produced by various cocaine pretreatment regimens in rats. Pharmacol Biochem Behav, 2000. 66(4): p. 765-70.

108. Johanson, C.E., R.L. Balster, and K. Bonese, Self-administration of psychomotor stimulant drugs: the effects of unlimited access. Pharmacol Biochem Behav, 1976. 4(1): p. 45-51.

109. Abuse, N.I.o.D., Research Monograph Series (88): Mechanisms of Cocaine Abuse and Toxcity, 1988, Public Health Services: Rockville, MD. p. 107-124.

110. Bedford, J.A., L.P. Bailey, and M.C. Wilson, Cocaine reinforced progressive ratio performance in the rhesus monkey. Pharmacol Biochem Behav, 1978. 9(5): p. 631-8.

111. Richardson, N.R. and D.C. Roberts, Progressive ratio schedules in drug selfadministration studies in rats: a method to evaluate reinforcing efficacy. $\mathrm{J}$ Neurosci Methods, 1996. 66(1): p. 1-11.

112. Depoortere, R.Y., et al., Parameters of self-administration of cocaine in rats under a progressive-ratio schedule. Pharmacol Biochem Behav, 1993. 45(3): $\mathrm{p}$. $539-48$.

113. Witkin, J.M. and S.R. Goldberg, Effects of cocaine on locomotor activity and schedule-controlled behaviors of inbred rat strains. Pharmacol Biochem Behav, 1990. 37(2): p. 339-42.

114. Calipari, E.S., et al., Intermittent cocaine self-administration produces sensitization of stimulant effects at the dopamine transporter. J Pharmacol Exp Ther, 2014. 349(2): p. 192-8.

115. Maier, E.Y., et al., The missing variable: ultrasonic vocalizations reveal hidden sensitization and tolerance-like effects during long-term cocaine administration. Psychopharmacology (Berl), 2012. 219(4): p. 1141-52. 
116. Kuz'min, A.V. and E.E. Evartau, [The intravenous self-administration of narcotics in mice]. Zh Vyssh Nerv Deiat Im I P Pavlova, 1991. 41(6): p. 1253-60.

117. Calipari, E.S., et al., Brief Intermittent Cocaine Self-Administration and Abstinence Sensitizes Cocaine Effects on the Dopamine Transporter and Increases Drug Seeking. Neuropsychopharmacology, 2014.

118. Feltenstein, M.W. and R.E. See, Potentiation of cue-induced reinstatement of cocaine-seeking in rats by the anxiogenic drug yohimbine. Behav Brain Res, 2006. 174(1): p. 1-8.

119. Thomsen, M. and S.B. Caine, Chronic intravenous drug self-administration in rats and mice. Curr Protoc Neurosci, 2005. Chapter 9: p. Unit 920.

120. Bouayad-Gervais, K., et al., The self-administration of rapidly delivered cocaine promotes increased motivation to take the drug: contributions of prior levels of operant responding and cocaine intake. Psychopharmacology (Berl), 2014. 231(21): p. 4241-52.

121. Minogianis, E.A., D. Levesque, and A.N. Samaha, The speed of cocaine delivery determines the subsequent motivation to self-administer the drug. Neuropsychopharmacology, 2013. 38(13): p. 2644-56.

122. Ward, A.S., et al., Binge cocaine self-administration in humans: intravenous cocaine. Psychopharmacology (Berl), 1997. 132(4): p. 375-81.

123. Kumor, K.M., et al., Subjective response during continuous infusion of cocaine. Pharmacol Biochem Behav, 1989. 33(2): p. 443-52.

124. Zhang, H., T.E. Prisinzano, and M.D. Donovan, Permeation and metabolism of cocaine in the nasal mucosa. Eur J Drug Metab Pharmacokinet, 2012. 37(4): p. 255-62.

125. Cone, E.J., Recent discoveries in pharmacokinetics of drugs of abuse. Toxicol Lett, 1998. 102-103: p. 97-101.

126. Shahi, K. and M. Baudry, Increasing binding affinity of agonists to glutamate receptors increases synaptic responses at glutamatergic synapses. Proc Natl Acad Sci U S A, 1992. 89(15): p. 6881-5.

127. Post, R.M., et al., Drug-environment interaction: context dependency of cocaineinduced behavioral sensitization. Life Sci, 1981. 28(7): p. 755-60.

128. Hoffman, D.C. and R.A. Wise, Locomotor-activating effects of the D2 agonist bromocriptine show environment-specific sensitization following repeated injections. Psychopharmacology (Berl), 1992. 107(2-3): p. 277-84. 
129. Hu, G.Y. and J.F. Storm, Excitatory amino acids acting on metabotropic glutamate receptors broaden the action potential in hippocampal neurons. Brain Res, 1991. 568(1-2): p. 339-44.

130. Crawford, D.C., et al., Calcium-independent inhibitory G-protein signaling induces persistent presynaptic muting of hippocampal synapses. J Neurosci, 2011. 31(3): p. 979-91.

131. Zhou, Z., et al., GluA2 (GluR2) regulates metabotropic glutamate receptordependent long-term depression through $N$-cadherin-dependent and cofilinmediated actin reorganization. J Neurosci, 2011. 31(3): p. 819-33.

132. Schmidt, H.D. and R.C. Pierce, Cocaine-induced neuroadaptations in glutamate transmission: potential therapeutic targets for craving and addiction. Ann N Y Acad Sci, 2010. 1187: p. 35-75.

133. Burgoyne, R.D., M.E. Graham, and M. Cambray-Deakin, Neurotrophic effects of NMDA receptor activation on developing cerebellar granule cells. J Neurocytol, 1993. 22(9): p. 689-95.

134. Scannevin, R.H. and R.L. Huganir, Postsynaptic organization and regulation of excitatory synapses. Nat Rev Neurosci, 2000. 1(2): p. 133-41.

135. Hung, A.Y., et al., Degradation of postsynaptic scaffold GKAP and regulation of dendritic spine morphology by the TRIM3 ubiquitin ligase in rat hippocampal neurons. PLoS One, 2010. 5(3): p. e9842.

136. Manneville, J.B., M. Jehanno, and S. Etienne-Manneville, Dlgl binds GKAP to control dynein association with microtubules, centrosome positioning, and cell polarity. J Cell Biol, 2010. 191(3): p. 585-98.

137. Haraguchi, K., et al., The hDLG-associated protein DAP interacts with dynein light chain and neuronal nitric oxide synthase. Genes Cells, 2000. 5(11): p. 905911.

138. Shin, S.M., et al., GKAP orchestrates activity-dependent postsynaptic protein remodeling and homeostatic scaling. Nat Neurosci, 2012. 15(12): p. 1655-66.

139. Welch, J.M., D. Wang, and G. Feng, Differential mRNA expression and protein localization of the SAP90/PSD-95-associated proteins (SAPAPS) in the nervous system of the mouse. J Comp Neurol, 2004. 472(1): p. 24-39.

140. Zuchner, S., et al., Multiple rare SAPAP3 missense variants in trichotillomania and OCD. Mol Psychiatry, 2009. 14(1): p. 6-9. 
141. Wan, Y., G. Feng, and N. Calakos, Sapap3 deletion causes mGluR5-dependent silencing of AMPAR synapses. J Neurosci, 2011. 31(46): p. 16685-91.

142. Timmer, K.M. and J.D. Steketee, Examination of a role for metabotropic glutamate receptor 5 in the medial prefrontal cortex in cocaine sensitization in rats. Psychopharmacology (Berl), 2012. 221(1): p. 91-100.

143. Tu, J.C., et al., Coupling of mGluR/Homer and PSD-95 complexes by the Shank family of postsynaptic density proteins. Neuron, 1999. 23(3): p. 583-92.

144. Gould, A.T., et al., Cocaine-elicited imbalances in ventromedial prefrontal cortex Homer1 versus Homer2 expression: implications for relapse. Addict Biol, 2015. 20(1): p. 148-57.

145. Zhang, G.C., et al., In vivo regulation of Homerla expression in the striatum by cocaine. Mol Pharmacol, 2007. 71(4): p. 1148-58.

146. Schenk, S. and S. Snow, Sensitization to cocaine's motor activating properties produced by electrical kindling of the medial prefrontal cortex but not of the hippocampus. Brain Res, 1994. 659(1-2): p. 17-22.

147. Ramos, M., B. Goni-Allo, and N. Aguirre, Ibotenic acid lesions of the medial prefrontal cortex block the development and expression of 3,4methylenedioxymethamphetamine-induced behavioral sensitization in rats. Behav Brain Res, 2005. 160(2): p. 304-11.

148. Steketee, J.D. and C.E. Beyer, Injections of baclofen into the ventral medial prefrontal cortex block the initiation, but not the expression, of cocaine sensitization in rats. Psychopharmacology (Berl), 2005. 180(2): p. 352-8.

149. Lecca, D., et al., Differential neurochemical and behavioral adaptation to cocaine after response contingent and noncontingent exposure in the rat. Psychopharmacology (Berl), 2007. 191(3): p. 653-67.

150. Kau, K.S., et al., Blunted cystine-glutamate antiporter function in the nucleus accumbens promotes cocaine-induced drug seeking. Neuroscience, 2008. 155(2): p. 530-7.

151. Steketee, J.D., Cocaine-induced behavioral sensitization is associated with increased protein kinase $C$ activity in the ventral tegmental area. Neuroscience Research Communications, 1997. 20(1): p. 59-67.

152. Rodriguez-Espinosa, N. and E. Fernandez-Espejo, Effects of acute and repeated cocaine on markers for neural plasticity within the mesolimbic system in rats. Psychopharmacology (Berl), 2015. 232(1): p. 57-62. 
153. Boudreau, A.C., et al., Signaling pathway adaptations and novel protein kinase A substrates related to behavioral sensitization to cocaine. J Neurochem, 2009.

110(1): p. 363-77.

154. Ghasemzadeh, M.B., P. Vasudevan, and C. Mueller, Locomotor sensitization to cocaine is associated with distinct pattern of glutamate receptor trafficking to the postsynaptic density in prefrontal cortex: early versus late withdrawal effects. Pharmacol Biochem Behav, 2009. 92(3): p. 383-92.

155. Pierce, R.C., et al., Repeated cocaine augments excitatory amino acid transmission in the nucleus accumbens only in rats having developed behavioral sensitization. J Neurosci, 1996. 16(4): p. 1550-60.

156. Pierce, R.C., et al., Ibotenic acid lesions of the dorsal prefrontal cortex disrupt the expression of behavioral sensitization to cocaine. Neuroscience, 1998. 82(4): p. 1103-14.

157. Lu, L., et al., Incubation of cocaine craving after withdrawal: a review of preclinical data. Neuropharmacology, 2004. 1: p. 214-26.

158. Boileau, I., et al., Modeling sensitization to stimulants in humans: an [11C] raclopride/positron emission tomography study in healthy men. Arch Gen Psychiatry, 2006. 63(12): p. 1386-95.

159. Renthal, W., et al., Histone deacetylase 5 epigenetically controls behavioral adaptations to chronic emotional stimuli. Neuron, 2007. 56(3): p. 517-29.

160. Kouzarides, T., Chromatin modifications and their function. Cell, 2007. 128(4): p. 693-705.

161. Yang, S., et al., Lower glutamate levels in rostral anterior cingulate of chronic cocaine users - A (1)H-MRS study using TE-averaged PRESS at 3 T with an optimized quantification strategy. Psychiatry Res, 2009. 174(3): p. 171-6.

162. Tang, W.X., et al., Molecular profiling of midbrain dopamine regions in cocaine overdose victims. J Neurochem, 2003. 85(4): p. 911-24.

163. Gipson, C.D. and P.W. Kalivas, More cocaine-more glutamate-more addiction. Biol Psychiatry, 2014. 76(10): p. 765-6.

164. Dahlin SL, H.X., Xue C-J, Wolf ME, Lesions of the prefrontal cortex or amygdala, but not fimbria fornix, prevent sensitization of amphetamine-stimulated horizontal activity. Soc. Neurosci. Abstr., 1994(20): p. 1621.

165. Karler, R., L.D. Calder, and S.A. Turkanis, DNQX blockade of amphetamine behavioral sensitization. Brain Res, 1991. 552(2): p. 295-300. 
166. Ferrario, C.R., et al., Neural and Behavioral Plasticity Associated with the Transition from Controlled to Escalated Cocaine Use. Biol Psychiatry. 58(9): p. 751-759.

167. Bartlett, E., et al., Selective sensitization to the psychosis-inducing effects of cocaine: a possible marker for addiction relapse vulnerability? Neuropsychopharmacology, 1997. 16(1): p. 77-82.

168. Sherer, M.A., Intravenous cocaine: psychiatric effects, biological mechanisms. Biol Psychiatry, 1988. 24(8): p. 865-85.

169. Morani, A.S., V. Panwar, and K. Grasing, Tactile hallucinations with repetitive movements following low-dose cocaine: implications for cocaine reinforcement and sensitization: case report. Am J Addict, 2013. 22(2): p. 181-2.

170. Kalivas, P.W., et al., A role for sensitization in craving and relapse in cocaine addiction. J Psychopharmacol, 1998. 12(1): p. 49-53.

171. Blanco, E., et al., Cocaine-induced behavioral sensitization is associated with changes in the expression of endocannabinoid and glutamatergic signaling systems in the mouse prefrontal cortex. Int J Neuropsychopharmacol, 2014. 18(1).

172. Alcantara, A.A., et al., Cocaine- and morphine-induced synaptic plasticity in the nucleus accumbens. Synapse, 2011. 65(4): p. 309-20.

173. Sorg, B.A., et al., Repeated daily cocaine alters subsequent cocaine-induced increase of extracellular dopamine in the medial prefrontal cortex. J Pharmacol Exp Ther, 1997. 281(1): p. 54-61.

174. Beyer, C.E. and J.D. Steketee, Dopamine depletion in the medial prefrontal cortex induces sensitized-like behavioral and neurochemical responses to cocaine. Brain Res, 1999. 833(2): p. 133-41.

175. Li, Y., et al., Both glutamate receptor antagonists and prefrontal cortex lesions prevent induction of cocaine sensitization and associated neuroadaptations. Synapse, 1999. 34(3): p. 169-80.

176. Colwell, C.S. and M.S. Levine, Metabotropic glutamate receptors modulate Nmethyl-D-aspartate receptor function in neostriatal neurons. Neuroscience, 1994. 61(3): p. 497-507.

177. Wolf, M.E. and K.Y. Tseng, Calcium-permeable AMPA receptors in the VTA and nucleus accumbens after cocaine exposure: when, how, and why? Front Mol Neurosci, 2012. 5: p. 72. 
178. Churchill, L., et al., Repeated cocaine alters glutamate receptor subunit levels in the nucleus accumbens and ventral tegmental area of rats that develop behavioral sensitization. J Neurochem, 1999. 72(6): p. 2397-403.

179. Swanson, C.J., et al., Repeated cocaine administration attenuates group I metabotropic glutamate receptor-mediated glutamate release and behavioral activation: a potential role for Homer. J Neurosci, 2001. 21(22): p. 9043-52.

180. Lu, W., L.M. Monteggia, and M.E. Wolf, Withdrawal from repeated amphetamine administration reduces NMDAR1 expression in the rat substantia nigra, nucleus accumbens and medial prefrontal cortex. Eur J Neurosci, 1999. 11(9): p. 3167-77.

181. Scheyer, A.F., M.E. Wolf, and K.Y. Tseng, A protein synthesis-dependent mechanism sustains calcium-permeable AMPA receptor transmission in nucleus accumbens synapses during withdrawal from cocaine self-administration. J Neurosci, 2014. 34(8): p. 3095-100.

182. Takuma, H., et al., Reduction of GluR2 RNA editing, a molecular change that increases calcium influx through AMPA receptors, selective in the spinal ventral gray of patients with amyotrophic lateral sclerosis. Annals of Neurology, 1999. 46(6): p. 806-815.

183. Monyer, H., et al., Heteromeric NMDA receptors: molecular and functional distinction of subtypes. Science, 1992. 256(5060): p. 1217-21.

184. Ryan, T.J. and S.G. Grant, The origin and evolution of synapses. Nat Rev Neurosci, 2009. 10(10): p. 701-12.

185. Yashiro, K. and B.D. Philpot, Regulation of NMDA receptor subunit expression and its implications for LTD, LTP, and metaplasticity. Neuropharmacology, 2008. 55(7): p. 1081-94.

186. Resink, A., et al., Agonist-induced down-regulation of NMDA receptors in cerebellar granule cells in culture. Eur J Neurosci, 1995. 7(8): p. 1700-6.

187. Li, X., et al., D-serine-induced inactivation of NMDA receptors in cultured rat hippocampal neurons expressing NR2A subunits is Ca2+-dependent. CNS Neurosci Ther, 2014. 20(11): p. 951-60.

188. von Engelhardt, J., et al., Synaptic NR2A- but not NR2B-Containing NMDA Receptors Increase with Blockade of Ionotropic Glutamate Receptors. Front Mol Neurosci, 2009. 2: p. 19. 
189. Huh, K.H. and R.J. Wenthold, Turnover analysis of glutamate receptors identifies a rapidly degraded pool of the $N$-methyl-D-aspartate receptor subunit, $N R 1$, in cultured cerebellar granule cells. J Biol Chem, 1999. 274(1): p. 151-7.

190. Hosokawa, T., et al., Stoichiometry and Phosphoisotypes of Hippocampal AMPAType Glutamate Receptor Phosphorylation. Neuron, 2014.

191. Huang, C.C., H.J. Lin, and K.S. Hsu, Repeated cocaine administration promotes long-term potentiation induction in rat medial prefrontal cortex. Cereb Cortex, 2007. 17(8): p. 1877-88.

192. Cass, D.K., et al., Developmental disruption of gamma-aminobutyric acid function in the medial prefrontal cortex by noncontingent cocaine exposure during early adolescence. Biol Psychiatry, 2013. 74(7): p. 490-501.

193. Ben-Shahar, O., et al., Extended daily access to cocaine results in distinct alterations in Homer $1 \mathrm{~b} / \mathrm{c}$ and NMDA receptor subunit expression within the medial prefrontal cortex. Synapse, 2009. 63(7): p. 598-609.

194. Roberts, P.J. and S.W. Davies, Excitatory receptors and their role in excitotoxicity. Biochem Soc Trans, 1987. 15(2): p. 218-9.

195. McGregor, A., G. Baker, and D.C. Roberts, Effect of 6-hydroxydopamine lesions of the medial prefrontal cortex on intravenous cocaine self-administration under a progressive ratio schedule of reinforcement. Pharmacol Biochem Behav, 1996. 53(1): p. 5-9.

196. Dalley, J.W., et al., Effects of excitotoxic lesions of the rat prefrontal cortex on $C R E B$ regulation and presynaptic markers of dopamine and amino acid function in the nucleus accumbens. Eur J Neurosci, 1999. 11(4): p. 1265-74.

197. Timmer, K.M. and J.D. Steketee, Group I metabotropic glutamate receptors in the medial prefrontal cortex: role in mesocorticolimbic glutamate release in cocaine sensitization. Synapse, 2013. 67(12): p. 887-96.

198. Iversen, S.D., Behavioural effects of manipulation of basal ganglia neurotransmitters. Ciba Found Symp, 1984. 107: p. 183-200.

199. Astrup, P. and I. Munkvad, [Glutamine and glutamic acid metabolism, with special reference to nervous tissue and psychiatric disease]. Nord Med, 1951. 45(4): p. 117-21.

200. Delay, J., et al., [Glutamic acid in psychiatry]. Sem Hop, 1951. 27(49): p. 214354. 
201. Sanberg, P.R. and J.T. Coyle, Scientific approaches to Huntington's disease. CRC Crit Rev Clin Neurobiol, 1984. 1(1): p. 1-44.

202. Deakin, J.F., et al., Frontal cortical and left temporal glutamatergic dysfunction in schizophrenia. J Neurochem, 1989. 52(6): p. 1781-6.

203. Palsson, E., et al., Markers of glutamate signaling in cerebrospinal fluid and serum from patients with bipolar disorder and healthy controls. Eur Neuropsychopharmacol, 2014.

204. Parikh, V., et al., Cocaine-induced neuroadaptations in the dorsal striatum: glutamate dynamics and behavioral sensitization. Neurochem Int, 2014. 75: p. 5465.

205. Hohnke, C.D., S. Oray, and M. Sur, Activity-dependent patterning of retinogeniculate axons proceeds with a constant contribution from AMPA and NMDA receptors. J Neurosci, 2000. 20(21): p. 8051-60.

206. Lin, Y., et al., Postsynaptic density protein-95 regulates NMDA channel gating and surface expression. J Neurosci, 2004. 24(45): p. 10138-48.

207. Ghasemzadeh, M.B., et al., Neuroadaptations in ionotropic and metabotropic glutamate receptor $m R N A$ produced by cocaine treatment. J Neurochem, 1999. 72(1): p. 157-65.

208. O'Connor, E.C., S. Bariselli, and C. Bellone, Synaptic basis of social dysfunction: a focus on postsynaptic proteins linking group-I mGluRs with AMPARs and NMDARs. Eur J Neurosci, 2014. 39(7): p. 1114-29.

209. Chen, M., et al., Sapap3 deletion anomalously activates short-term endocannabinoid-mediated synaptic plasticity. J Neurosci, 2011. 31(26): p. 956373. 


\section{VITA}

Kyle Christopher Summers was born in Memphis, Tennessee in 1986. He earned a Bachelors of Science in Biology from Christian Brothers University in May of 2008, and will be receiving his Ph.D. in Biomedical Sciences with a concentration in Neuroscience from the University of Tennessee Health Science Center in August of 2015 